medRxiv preprint doi: https://doi.org/10.1101/2021.07.28.21261230; this version posted July 31, 2021. The copyright holder for this preprint (which was not certified by peer review) is the author/funder, who has granted medRxiv a license to display the preprint in perpetuity. It is made available under a CC-BY-NC-ND 4.0 International license .

\title{
Identification of muscle weakness in older adults from normalized lower and upper limbs strength
}

Pedro Pugliesi Abdalla ${ }^{12^{*}}$, Lucimere Bohn ${ }^{2}$, Leonardo Santos Lopes da Silva ${ }^{3}$, André Pereira dos Santos ${ }^{1}$, Marcio Fernando Tasinafo Junior ${ }^{3}$, Ana Claudia Rossini Venturini ${ }^{1}$, Anderson dos Santos Carvalho ${ }^{4}$, David Martinez Gomez ${ }^{5}$, Jorge Mota ${ }^{2}$, Dalmo Roberto Lopes Machado ${ }^{123}$.

${ }^{1}$ University of São Paulo, College of Nursing at Ribeirão Preto, Ribeirão Preto, Brazil.

${ }^{2}$ University of Porto, Faculty of Sports, Porto, Portugal.

${ }^{3}$ University of São Paulo, School of Physical Education and Sport of Ribeirão Preto, Ribeirão Preto, Brazil.

${ }^{4}$ Paulista University, Physical Education Course, São José do Rio Preto, Brazil.

${ }^{5}$ Autonomous University of Madrid, School of Medicine, Madrid, Spain.

*Corresponding author: Pedro Pugliesi Abdalla (pedroabdalla11 @gmail.com)

Avenue of Bandeirantes no 3900, University Campus - Monte Alegre, Ribeirão Preto-SP, Brazil.

Zip code: $14040-902$

Tel: +55 $163315-3468$

Acknowledgements

Nothing to declare.

Declaration of Conflicting Interests

The Author(s) declare(s) that there is no conflict of interest.

Funding

This work was supported by the Conselho Nacional de Desenvolvimento Científico e Tecnológico (CNPq) [through PhD scholarship number 142248/2018-5 granted to Pedro Pugliesi Abdalla]; and the Coordenação de Aperfeiçoamento de Pessoal de Nível Superior (CAPES) [finance Code 001, through PhD scholarship number 88887.593242/2020-00 granted to Pedro Pugliesi Abdalla, and through scholarship number 88887.371516/2019-00 granted to Dalmo Roberto Lopes Machado]. The funding body had no role in the design of the study and collection, analysis, and interpretation of data and in writing the manuscript. 
medRxiv preprint doi: https://doi.org/10.1101/2021.07.28.21261230; this version posted July 31, 2021. The copyright holder for this preprint (which was not certified by peer review) is the author/funder, who has granted medRxiv a license to display the preprint in perpetuity.

It is made available under a CC-BY-NC-ND 4.0 International license .

\begin{abstract}
Introduction: Weakness is a natural age-related condition meaning the loss of muscle strength that impairs older adults' mobility and quality of life. Because the relationship between muscle strength and body-size variables is non-linear, weakness is misclassified in older adults with extreme body size (e.g., light, short, heavy, or tall). This misclassification can be overcome using the allometric approach. Objectives: To propose cut-off points for older adults' weakness for upper and lower limbs muscle strength normalized by body size with the ratio standard and allometric scaling. Methods: Ninety-four community-dwelling older adults (69.1\% women) were assessed for 49 body-size variables (anthropometry, body composition and body indexes), handgrip strength (HGS), one maximum repetition measurement for knee extensors $\left(1 \mathrm{RM}_{\mathrm{knee}}\right.$ extensors), isokinetic knee extension peak torque at $60 \%$ s (knee extension $\mathrm{PT}^{60 \%}$ ), and six-minute walk test (6MWT). Ratio standard (muscle strength/body size) and allometric scaling (muscle strength/body size ${ }^{\mathrm{b}}$; when ${ }^{\mathrm{b}}$ is the allometric exponent) were applied for body-size variables that significantly were correlated with HGS, $1 \mathrm{RM}_{\text {knee extensors }}$ and knee extension $\mathrm{PT}^{60 \%}$. Cut-off points were computed based on ROC curve and Youden index. When there was mobility limitation $(6 \mathrm{MWT}<400 \mathrm{~m})$ cut-off were computed according to sex. Results: Absolute HGS, 1 RM $\mathrm{knee}_{\mathrm{k}}$ extensors and knee extension $\mathrm{PT}^{60 \%}$ cut-off points were not adequate because they were associated with body size ( $>0.30)$. But it was corrected with muscle strength normalization according to body size-variables: HGS $(n=1) ; 1 \mathrm{RM}_{\text {knee extensors }}(\mathrm{n}=24)$ and knee extension $\mathrm{PT}^{60 \% \mathrm{~s}}(\mathrm{n}=24)$. The best cutoff points, with the highest area under the curve (AUC), were found after normalization for men: HGS/forearm circumference $(1.33 \mathrm{~kg} / \mathrm{cm}, \mathrm{AUC}=0.74), 1 \mathrm{RM}_{\text {knee extensors }} /$ triceps skinfold (4.22 kg/mm, AUC=0.81), and knee extension $\mathrm{PT}^{60^{\circ} / \mathrm{s}} /$ body mass*height ${ }^{0.43}\left(13.0 \mathrm{Nm} / \mathrm{kg}^{*} \mathrm{~m}^{0.43}\right.$, $\mathrm{AUC}=0.94)$; and for women: $\mathrm{HGS} /$ forearm circumference $(1.04 \mathrm{~kg} / \mathrm{cm}, \mathrm{AUC}=0.70), 1 \mathrm{RM}_{\mathrm{knee}}$ extensors/body mass $(0.54 \mathrm{~kg} / \mathrm{kg}, \mathrm{AUC}=0.76)$; and knee extension $\mathrm{PT}^{60 \%} / \mathrm{s} /$ body mass ${ }^{0.72}(3.14$ $\mathrm{Nm} / \mathrm{kg}^{0.72}$; AUC=0.82). Conclusion: Normalization removes the effect of extreme body size on muscle strength and improves the accuracy to identify weakness at population level, reducing the risk of false-positive cases.
\end{abstract}

Keywords: Allometrically scaled, Dynapenia, Elderly, Frailty, Sarcopenia, Scaling.

\title{
Highlights
}

- This study proposed a new approach to identify muscle weakness in older adults based on upper and lower limbs muscle strength normalized by body size (ratio standard and allometry). 
medRxiv preprint doi: https://doi.org/10.1101/2021.07.28.21261230; this version posted July 31, 2021. The copyright holder for this preprint (which was not certified by peer review) is the author/funder, who has granted medRxiv a license to display the preprint in perpetuity.

It is made available under a CC-BY-NC-ND 4.0 International license .

- The identification of functional limitation is more precise when procedures of muscle strength normalization is applied.

\section{INTRODUCTION}

Muscle weakness is a natural muscle strength loss occurring along aging, and it predicts older adults' increased risk of hospital admissions, depression, fractures and premature mortality [1-3]. Muscle weakness can predict functional disability (i.e., difficulty to perform instrumental and basic activities of daily living-ADL) like as mobility limitation [4], which is even more important than multimorbidity to forecast mortality amongst older adults [5]. As a consequence of its predictive ability, muscle weakness was used to identify geriatric syndromes such as dynapenia [6], frailty [7] and sarcopenia [8].

Muscle weakness is normally measured using muscle strength tests such as handgrip (HGS) or leg extension strength [8]. The current values to identify muscle weakness are based on absolute (non-normalized) muscle strength results [7, 9-17] or dividing absolute results by some (i.e., body mass) body-size variable (ratio standard) $[18,19]$. The identification of weakness based on absolute muscle strength cut-off points may be inaccurate for lighter body mass and shorter height older adults [20-22]. In fact, the absolute values characterize lighter and shorter body size older adults as having muscle weakness, even if they sustain their instrumental and basic ADL [23]. This is a false positive muscle weakness diagnostic, that frequently leads to an unnecessarily utilization of public health resources, contributing to health burden [24]. Another topic that merits consideration is the inaccuracy of the ratio standard procedure because it overestimates the real strength of light/short older adults and underestimates it for tall/heavy ones [23]. These limitations are a consequence of the nonlinear relationship between muscle strength and body-size variables [20-22]. To overcome these constraints, the utilization of allometric scaling, that contemplates power and sensitivity in the nonlinear relationship between muscle strength and body size with the allometric exponent $\left({ }^{b}\right)$ might represent an adequate option [20-23].

Previous studies reported already the power function ratio in older adults between HGS and body-size variables as body mass $\left({ }^{b=0.63}\right.$ or ${ }^{0.40}$ or $\left.{ }^{0.31}\right)$ [20-22], height $\left({ }^{b=1.84}\right)$ [21] and fatfree mass $(\mathrm{FFM})\left({ }^{\mathrm{b}=0.46}\right)[21]$ and between leg extension strength and body mass $\left({ }^{\mathrm{b}=0.67}\right.$ or ${ }^{0.69}$ or 0.72 or ${ }^{0.74}$ or $\left.^{0.96}\right)[23,25]$. Indeed, scaling HGS by body size (example: HGS/heigth ${ }^{1.84}$ ) removes the effect of body size on muscle strength [21], but the scaling muscle strength by body size to determine muscle weakness cut-off points has not been considered from HGS and knee extension in isokinetic dynamometer, excepting the one maximum repetition measurement for 
medRxiv preprint doi: https://doi.org/10.1101/2021.07.28.21261230; this version posted July 31, 2021. The copyright holder for this preprint (which was not certified by peer review) is the author/funder, who has granted medRxiv a license to display the preprint in perpetuity.

It is made available under a CC-BY-NC-ND 4.0 International license .

knee extensors $\left(1 \mathrm{RM}_{\mathrm{knee}}\right.$ extensors $)$ scaled to body mass [23]. Besides, important body-size variables related to mobility and ADL (e.g. fat mas [26], FFM [27] and leg length [28]) were not utilized to scaling muscle strength and create muscle weakness cut-off points.

Thus, our objective is to propose cut-off points for older adults' weakness with upper and lower limbs muscle strength normalized by body-size with the ratio standard and allometric scaling. We hypothesize that the normalization of muscle strength by ratio standard and allometry can be a way to approach muscle strength regardless of body size, which should reduce the risk of bias in identifying false-positive cases of vulnerable older people.

\section{MATERIAL AND METHODS}

\section{Design and Study population}

This is a cross-sectional study conducted from October 2016 to May 2017 at the University Hospital of Ribeirao Preto School of Medicine, University of São Paulo, Brazil (HCFMRP-USP). The study was approved by the HC-FMRP-USP institutional review board. Older adults were voluntarily recruited and assigned an informed consent. This manuscript followed the guidelines from The Strengthening the Reporting of Observational Studies in Epidemiology (STROBE) conference list, and the completed checklist is attached.

The sample consisted of 94 community-dwelling older adults (>60 years old, $69.1 \%$ women) recruited in projects for older adults of USP and in health community services. Inclusion criteria were walk independently, absent limitation to execute all procedures, acute infections, cancer diagnosis, hip or knee prostheses, unstable cardiovascular condition, stroke sequelae, tumors, and weight loss $>3 \mathrm{~kg}$ in the last three months. The exclusion criteria were discontinuity in the study and cognition impairment.

A sample size calculation $\left(\mathrm{n}=[\mathrm{ZySD} / \varepsilon]^{2}\right)$ with trust level $(\mathrm{Zy}=0,95)$, greater compatible population variability founded in the literature (SD of $1 \mathrm{RM}_{\text {knee extensors }} \pm 19.96 \mathrm{~kg}$ ) $[29,30]$ and maximum desired error $(\varepsilon \leq 8.0 \mathrm{~kg})$ was performed and identified a minimum sample size of $\mathrm{n}=24$ for each sex.

\section{Procedures}

A multidisciplinary health team (nurses, nutritionists, pharmacists, physical education professors, physicians, and physiotherapists) performed data collection. The appraisers were the same in each test. Data collection occurred on three non-consecutive days: ${ }^{\text {st }}$ ) recruitment: inclusion criteria verification and cognition assessment; by phone calls (inclusion criteria) or face to face (cognition assessment) of older adults who agreed to participate in the study; $2^{\text {nd }}$ ) 
medRxiv preprint doi: https://doi.org/10.1101/2021.07.28.21261230; this version posted July 31, 2021. The copyright holder for this preprint (which was not certified by peer review) is the author/funder, who has granted medRxiv a license to display the preprint in perpetuity. It is made available under a CC-BY-NC-ND 4.0 International license .

anthropometrics, body composition, HGS, mobility and physical activity level assessment; and $3^{\text {rd }}$ ) lower limbs muscle strength assessment.

\section{Cognition Assessment}

The validated Mini Mental State Examination (MMSE) was used to assess participants' cognition status [31]. Those who have MMSE $\leq 12$ were excluded [32].

\section{Measure of Body-Size Variables}

Forty-nine body-size variables (SUPPLEMENT A) were collected to propose allometric exponents and to normalize performance in muscle strength tests. The selection of these variables were based on those previously used to calculate body indices [33-41], and involved anthropometric measurements [42] and body composition (Dual Energy X-ray AbsorptiometryDXA and bioelectrical impedance analysis-BIA), as briefly detailed below.

\section{Body Indexes}

The body indexes derived from anthropometry were body mass index (BMI, $\mathrm{kg} / \mathrm{m}^{2}$ ) [33], body mass*height [34], human body surface area (SA, $\mathrm{m}^{2}$ ) [35], absolute mid-arm muscle circumference (MAMC, cm) [36], corrected arm muscle area (CAMA, cm) [37], arm fat area $\left(\mathrm{AFA}, \mathrm{cm}^{2}\right)$ [38], FFM [39] and fat mass (obtained by body mass difference). The body indexes derived from body composition were lean soft tissue (LST) of arms and legs, appendicular skeletal muscle mass (ASM), ASM/height (m)2 [40], FFM (estimated from BIA) [41] and DXA, when fat mass were estimated by body mass difference.

\section{Mobility Measurement}

The cut-off points for muscle weakness were established based on mobility limitation. Mobility performance was verified based on the six-minute walk test (6MWT) carried out in a corridor 30-meter length. Along this path, at every three meters there was a cone to help researcher to precisely identify the walked distance [28]. Participants were instructed to cover the longest distance walking as faster as they could during the six-minute time. Nevertheless, participants could slow down, interrupt the walking, and resume the test whenever desired, although time was not paused. Total walked distance was recorded and mobility disability was characterized when the 6MWT<400 m [43]. 
medRxiv preprint doi: https://doi.org/10.1101/2021.07.28.21261230; this version posted July 31, 2021. The copyright holder for this preprint (which was not certified by peer review) is the author/funder, who has granted medRxiv a license to display the preprint in perpetuity.

It is made available under a CC-BY-NC-ND 4.0 International license .

Muscle strength was measured using HGS, one maximum repetition measurement for knee extensors $\left(1 \mathrm{RM}_{\text {knee extensors }}\right)$ and isokinetic knee extension peak torque at a velocity of $60 \%$ (knee extension $\left.\mathrm{PT}^{60 \%}\right)$. The maximum HGS was measured with a manual dynamometer (Jamar®, model 5030J1) using a previously published protocol [44]. Three attempts were performed, one minute apart, with the dominant hand and the highest result was recorded in $\mathrm{kg}$ as HGS [45, 46]. The $1 R M_{\text {knee extensors }}$ was estimated in a leg extension machine (Lion Fitness ${ }^{\circledR}$ model LFS) with a submaximal repetition protocol: $1 \mathrm{RM}=$ weight lifted/(1.0278-[0.0278* $\mathrm{n}^{\mathrm{o}}$ of reps]) [47]. The detailed protocol was published elsewhere [23]. The isokinetic knee extension peak torque at $60 \%$ s (knee extension $\mathrm{PT}^{60 \%}$ ) of the right lower limb was recorded with the Biodex (model System 4 Pro) isokinetic dynamometer and results are in newton-meter ( $\mathrm{Nm})$ according to standardized protocol [48].

\section{Physical Activity Level Measurement}

The International Physical Activity Questionnaire - Short Version was used to get physical activity level [49]. Physical activity level was dichotomized into sedentary (0) and irregularly active, active or very active (1). These two categories were introduced in the models to provide allometric exponents.

\section{Muscle Strength Normalization Procedures (Ratio Standard and Allometric Scaling)}

HGS, $1 \mathrm{RM}_{\text {knee extensors }}$ and knee extension $\mathrm{PT}^{60 \% /}$ were considered in three different ways: 1) absolute (non-normalized); 2) ratio standard (muscle strength/body-size variable); and 3) allometrically adjusted (muscle strength/body-size variable ${ }^{\mathrm{b}}$ ).

Allometric exponents $\left(^{b}\right)$ were proposed only for body-size variables that showed significant correlation (Pearson's correlation) with muscle strength. To generate the allometric exponents, muscle strength (Y) and body-size variables $(\mathrm{X})$ were converted to natural logarithm (ln) and the slope of regression line is allometric exponent $\left({ }^{b}\right)$, according to more detail previously published [21]. Therefore, allometric exponents were discarded when the interaction (ln body-size variable*age*sex*physical activity level) was significant or when there was multicollinearity in the linear regression (variance inflation factor [VIF]>10) [50].

We also consider other allometric exponents $\left({ }^{b}\right)$ of the literature, as described in Table 1. 
medRxiv preprint doi: https://doi.org/10.1101/2021.07.28.21261230; this version posted July 31, 2021. The copyright holder for this preprint (which was not certified by peer review) is the author/funder, who has granted medRxiv a license to display the preprint in perpetuity. It is made available under a CC-BY-NC-ND 4.0 International license .

In order to verify whether normalization removed the influence of body size on muscle strength, the correlation between normalized muscle strength and body-size variables (body mass, height and body-size used) should be negligible ( $r \leq 30)$ [51].

\section{Statistical Analysis}

\section{Proposition of Cut-off Points for Muscle Weakness}

Absolute muscle strength and normalized by ratio standard or allometric scaling had their area under the curve (AUC) quantified by the ROC curve. The Youden index [52] selected the most appropriate cut-off points with the best relationship between sensitivity and specificity for the primary outcome (functional limitation: 6MWD<400) [43].

The cut-off points were considered adequate when they have $\mathrm{AUC} \geq 0.70$ [53] simultaneously for both sexes $(\mathrm{p}<0.05)$ and when the correlation between muscle strength and body-size variables (body mass, height and body-size used) were negligible $(r \leq 0.30)$ [51].

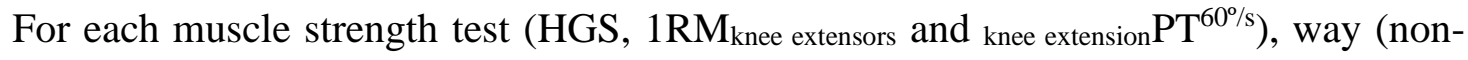
normalized, ratio standard and allometric scaling) and for each and sex was selected the adequate cut-off point according the superior accuracy. When there was a tie in accuracy, the variable with the greatest sensitivity or specificity was chosen. Finally, the AUC - ROC curves of non-normalized and normalized muscle strength were compared with each other to decide the best cut-off point.

The analyzes were performed using the SPSS 25.0 statistical package, and the ROC curves and Youden index in MedCalc 15.2 with a previously established level of significance $(\alpha=5 \%)$.

\section{RESULTS}

Sample was encompassed by 100 older adults (69 women) who agreed to participate in the study. From those, 6 were excluded for different reasons, as the stages of the study proceeded, as detailed in Figure 1. Therefore, the final sample comprised 29 older men (31\%) and 65 older women $(69 \%)$.

\section{<insert Figure 1>}

Sample characterization according to sex is shown in Table 2. Twenty-five women $(38.5 \%)$ and seven men $(24.1 \%)$ had functional limitation (6MWT<400 m). 
medRxiv preprint doi: https://doi.org/10.1101/2021.07.28.21261230; this version posted July 31, 2021. The copyright holder for this preprint (which was not certified by peer review) is the author/funder, who has granted medRxiv a license to display the preprint in perpetuity. It is made available under a CC-BY-NC-ND 4.0 International license .

The correlations between muscle strength and body-size variables are also shown in Table 2. Most of the body-size variables showed a significant correlation with muscle strength $(\mathrm{r}=-0.41$ to $0.75 ; \mathrm{p}<0.05)$.

\section{<insert Table 2>}

Allometric exponents were proposed for those body-size variables that showed a significant relationship with muscle strength (Table 2). Linear regressions to obtain allometric exponents are shown in SUPPLEMENT B. All regressions were significant to explain muscle strength $(\mathrm{p}<0.05)$, with adjusted $\mathrm{R}^{2}$ ranging from 0.39 to 0.61 . The regression coefficients $(\beta)$ obtained for each body-size variable represent the allometric exponents obtained. For HGS, the allometric exponents of triceps, pectoral, abdominal and thigh skinfolds were discarded because the interaction terms were statistically significant $(\mathrm{p}<0.05)$ and have accentuated multicollinearity (VIF>10). The remaining allometric exponents were used to perform normalization (for example, $1 \mathrm{RM}_{\text {knee extensors }} /$ body mass $^{0.44}$ ).

The sex-specific cut-off points proposed for HGS, $1 \mathrm{RM}_{\text {knee extensors }}$ and knee extension $\mathrm{PT}^{60 \%}$ (non-normalized, ratio standard and allometric scaling) to identify muscle weakness are presented in the SUPPLEMENT C. In the same supplement there are also presented correlations between muscle strength and body size (body mass, height and body-size variable used in normalization).

Non-normalized HGS, $1 \mathrm{RM}_{\text {knee extensors }}$ and knee extension $\mathrm{PT}^{60 \%}$ cut-off points to identify muscle weakness were not adequate for both sexes or because they did not present AUC $\geq 0.70$ $(\mathrm{p}<0.05)$ or because they had a significant association with body size $(r>0.30 ; p<0.05)$ (SUPPLEMENT C).

Table 3 shows the cut-off points based on the ratio standard and allometric scaling classified as adequate.

\section{<insert Table 3>}

A comparison of the most accurate ROC curves is presented in Figure 2 to support the decision for the best cut-off point between non-normalized, ratio standard and allometric scaling of HGS and lower limbs strength $\left(1 \mathrm{RM}_{\text {knee extensors }}\right.$ and knee extension $\left.\mathrm{PT}^{60 \%}\right)$ for each sex. 
medRxiv preprint doi: https://doi.org/10.1101/2021.07.28.21261230; this version posted July 31, 2021. The copyright holder for this preprint (which was not certified by peer review) is the author/funder, who has granted medRxiv a license to display the preprint in perpetuity.

It is made available under a CC-BY-NC-ND 4.0 International license .

For men, there were no differences in accuracy (AUC) to identify functional limitation between absolute muscle strength, normalized by ratio standard or by allometric scaling (p>0.05; Figure $2 \mathrm{a}, \mathrm{b}, \mathrm{c}$ ). However, the absolute muscle strengths (HGS, 1 RM $\mathrm{knee}_{\text {extensors }}$ and knee extension $\mathrm{PT}^{60 \%}$ ) previously indicated great dependence $(\mathrm{r}>0.30)$ on body size (SUPPLEMENT C), suggesting the need for normalization to avoid errors in the classification of weakness. The normalized muscle strength increased the AUC and made it possible to classify muscle weakness of older adults with extreme body sizes, independently.

For women, only after normalizing muscle strength the AUC values perform acceptable to identify functional limitation (AUC $>0.70$; Figure $2 \mathrm{~d}, \mathrm{e}$ ). The exception was knee extension $\mathrm{PT}^{60 \%}$, when the absolute values already had adequate accuracy (AUC>0.70), although without the desirable independence of body size. All the normalizations increased $\left({ }^{\star}\right)$ the AUC $(p<0.001)$.

\section{DISCUSSION}

Cut-off points based on upper and lower limbs muscle strength were proposed to identify muscle weakness in older adults of both sexes. The non-normalized cut-off points for HGS and lower limbs strength were significantly associated with body size, which involves biases to assess older adults with extreme body size (e.g., heavy or short). After normalizing HGS and lower limbs strength by the ratio standard or by the allometry, the association with body size was no longer relevant. In addition, for women, the accuracy to predict mobility limitation/muscle weakness from normalized muscle strength cut-off points become acceptable when compared to non-normalized strategy. In men, muscle strength normalization did not increase accuracy. However, all normalized models of both sexes avoided biases in the assessment of muscle weakness/mobility limitation, to isolate the natural interdependence between muscle strength and body size [21].

To the best of our knowledge, this is the first study to propose muscle weakness cut-off points for the HGS and knee extension $\mathrm{PT}^{60 \%}$ allometrically adjusted in older adults. In a previous study, $1 \mathrm{RM}_{\text {knee extensors }}$ was allometrically adjusted for body mass [23], but not according to all other potential body size variables. Indeed, we expanded the number of variables that can be

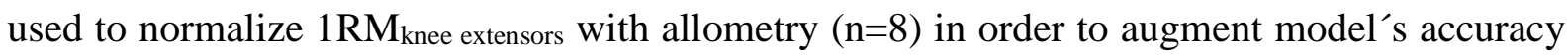
for identifying muscle weakness regardless of extreme body sizes. Other studies proposed muscle weakness cut-off points with HGS normalized by ratio standard (body mass or BMI) $[18,54,55]$ or stratified by BMI quartiles [7]. There are also muscle weakness cut-off points for knee extension $\mathrm{PT}^{60 \% /}$ normalized by body mass [19]. However, these studies did not compare the 
medRxiv preprint doi: https://doi.org/10.1101/2021.07.28.21261230; this version posted July 31, 2021. The copyright holder for this preprint (which was not certified by peer review) is the author/funder, who has granted medRxiv a license to display the preprint in perpetuity.

It is made available under a CC-BY-NC-ND 4.0 International license .

accuracy of normalized with non-normalized muscle strength to identify muscle weakness. Furthermore, they did not explore other body-size variables to normalize muscle strength.

Previous studies have proposed allometric exponents to normalize muscle strength, including HGS [20-22], $1 \mathrm{RM}_{\text {knee extensors }}$ [23], knee extension $\mathrm{PT}^{60 \% \mathrm{~s}}[25,34]$, and they are comparable with the ones found in the present study. Curvilinear (allometric) relationship variables is confirmed when allometric coefficient $\left({ }^{b}\right)$ is between 0.00 and 0.99 [56], while the linear relationship is characterized when the exponent is $\geq 1.00$ [56]. In the literature, body mass generally presents an allometric relationship with muscle strength independently of the test (HGS, $1 \mathrm{RM}_{\text {knee extensors }}$ or knee extension $\mathrm{PT}^{60 \%}$; Table 1), confirming our findings (SUPPLEMENT B), when ${ }^{b}$ exponents were 0.22 (HGS), 0.44 (1RM $\mathrm{R}_{\text {knee extensors) }}$ ) and 0.37 (knee extension $\mathrm{PT}^{60 \%}$ ). Contrarily, height tends to have a linear relationship $\left({ }^{b} \geq 1.00\right)$ with muscle strength [21], what was also confirmed by our proposed allometric exponents (SUPPLEMENT B), that were between 1.87 and 3.27 .

Some strengths of our study are noteworthy. We proposed muscle weakness cut-off points for isokinetic dynamometer, considered as a "gold standard" resource to assess lower limbs strength. The estimated $1 \mathrm{RM}_{\mathrm{knee}}$ extensors obtained with submaximal repetition protocol and the HGS are valid for older adults, even for those with muscle weakness [48, 57]. An extensive number of body-size variables $(n=49)$ were tested in our study, expanding the possibilities to promote the normalization of performance in muscle strength tests. Furthermore, regardless of the chosen muscle strength test to evaluate weakness, our findings can be applied with sufficient accuracy (AUC>0.70) both for scientific research (knee extension $\mathrm{PT}^{60 \%}$ ) and population-based

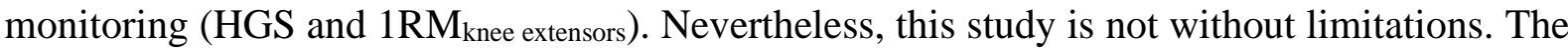
individual muscle strength decline along aging may have been underestimated with the crosssectional design. Another limitation is the small and local sample size of our study, requiring caution to extrapolate these findings inferentially to other populations.

We found greater accuracy (AUC) for normalized lower limbs strength (isokinetic dynamometer and leg extension machine) than manual dynamometer (normalized upper extremity strength), usually adopted to predict mobility limitations/muscle weakness [8]. However, the isokinetic dynamometer is expensive and generally more available in terms of research. Even though, our normalized models are also applicable in clinical practice from manual dynamometers (widely available in geriatric environments) and leg extension machines (available in most fitness centers, adequate environment for intervention against aged-related muscle weakness) [30]. The assessment of HGS and $1 \mathrm{RM}_{\text {knee extensors }}$ and proper classification of muscle weakness amongst older adults should be frequent in clinical practice to better target 
medRxiv preprint doi: https://doi.org/10.1101/2021.07.28.21261230; this version posted July 31, 2021. The copyright holder for this preprint (which was not certified by peer review) is the author/funder, who has granted medRxiv a license to display the preprint in perpetuity.

It is made available under a CC-BY-NC-ND 4.0 International license .

health expending, avoiding unnecessary expenditures. Future research should observe if proposed allometric exponents can be utilized to normalize muscle strength for different older adults' population, with other ethnicity/race characteristics.

As an applied example to avoid false positive diagnosis for muscle weakness, we hypothesize one older man with extreme lower values of body mass (42 kg), $1.57 \mathrm{~m}$ of height, who performed knee extension $\mathrm{PT}^{60 \%}$ of $85.2 \mathrm{Nm}$. If we consider our absolute cut-off point $(\leq 85.4$ $\mathrm{Nm}$ ), this older man has muscle weakness confirmed. However, when considered the normalized knee extension $\mathrm{PT}^{60 \%} /\left([\text { body mass } * \text { height }]^{0.43}\right)$, the adjusted value $(14.1 \mathrm{Nm} / \mathrm{kg} * \mathrm{~m})$ is above of the cut-off point (13.0 Nm/kg*m; Table 3). Normalization would also avoid false negative cases, for large body size of older adults. The mistaken framing of false positive weakness cases could greatly impact the financial resources in the health and older people care systems. Especially in low- and middle-income countries, where these resources are scarcer.

\section{CONCLUSION}

Upper and lower limbs muscle weakness cut-off points standardized according to body size were proposed for older adults of both sexes. The normalization has increased accuracy for identify women with muscle weakness; but not in men, whose absolute muscle strength values have an acceptable accuracy. However, normalization made muscle strength independent of body size, confirming our hypothesis and preventing bias in the evaluation of older adults with extreme body size (e.g., very low or very heavy). Forty-nine valid models were proposed for older adults of both sexes, with different possibilities of body's normalization of muscle strength, which broadens the interpretation of muscle strength with less risk of attributing a false-positive diagnosis to muscle weakness.

\section{REFERENCES}

1. Bohannon RW. Hand-grip dynamometry predicts future outcomes in aging adults. Journal of geriatric physical therapy (2001). 2008;31:3-10. doi:10.1519/00139143200831010-00002

2. Teng Z, Zhu Y, Yu X, Liu J, Long Q, Zeng Y, et al. An analysis and systematic review of sarcopenia increasing osteopenia risk. PloS one. 2021;16:e0250437.

doi:10.1371/journal.pone.0250437

3. Bohannon RW. Grip Strength: An Indispensable Biomarker For Older Adults. Clinical interventions in aging. 2019;14:1681-91. doi:10.2147/cia.s194543

4. Santanasto AJ, Miljkovic I, Cvejkus RK, Wheeler VW, Zmuda JM. Sarcopenia Characteristics Are Associated with Incident Mobility Limitations in African Caribbean Men: The Tobago Longitudinal Study of Aging. J Gerontol A Biol Sci Med Sci. 2020;75:1346-52. doi:10.1093/gerona/glz233 
medRxiv preprint doi: https://doi.org/10.1101/2021.07.28.21261230; this version posted July 31, 2021. The copyright holder for this preprint (which was not certified by peer review) is the author/funder, who has granted medRxiv a license to display the preprint in perpetuity.

5. Landi F, Liperoti R, Russo A, Capoluongo E, Barillaro C, Pahor M, et al. Disability, more than multimorbidity, was predictive of mortality among older persons aged 80 years and older. Journal of clinical epidemiology. 2010;63:752-9. doi:10.1016/j.jclinepi.2009.09.007 6. Clark BC, Manini TM. Sarcopenia $\neq$ Dynapenia. The Journals of Gerontology: Series A. 2008;63:829-34. doi:10.1093/gerona/63.8.829

7. Fried LP, Tangen CM, Walston J, Newman AB, Hirsch C, Gottdiener J, et al. Frailty in older adults evidence for a phenotype. J Gerontol A Biol Sci Med Sci. 2001;56:M146-M57. 8. Cruz-Jentoft AJ, Bahat G, Bauer J, Boirie Y, Bruyère O, Cederholm T, et al. Sarcopenia: revised European consensus on definition and diagnosis. Age Ageing. 2018;48:16-31. doi:10.1093/ageing/afy169

9. Dodds RM, Syddall HE, Cooper R, Benzeval M, Deary IJ, Dennison EM, et al. Grip strength across the life course: normative data from twelve British studies. PloS one. 2014;9:e113637. doi:10.1371/journal.pone.0113637

10. Lauretani F, Russo CR, Bandinelli S, Bartali B, Cavazzini C, Di Iorio A, et al. Ageassociated changes in skeletal muscles and their effect on mobility: an operational diagnosis of sarcopenia. Journal of Applied Physiology. 2003;95:1851-60.

11. Wang YC, Bohannon RW, Li X, Sindhu B, Kapellusch J. Hand-Grip Strength: Normative Reference Values and Equations for Individuals 18 to 85 Years of Age Residing in the United States. The Journal of orthopaedic and sports physical therapy. 2018;48:685-93. doi:10.2519/jospt.2018.7851

12. Albrecht BM, Stalling I, Bammann K. Sex- and age-specific normative values for handgrip strength and components of the Senior Fitness Test in community-dwelling older adults aged 65-75 years in Germany: results from the OUTDOOR ACTIVE study. BMC geriatrics. 2021;21:273. doi:10.1186/s 12877-021-02188-9

13. Hofmann M, Halper B, Oesen S, Franzke B, Stuparits P, Tschan H, et al. Serum concentrations of insulin-like growth factor-1, members of the TGF-beta superfamily and follistatin do not reflect different stages of dynapenia and sarcopenia in elderly women. Experimental gerontology. 2015;64:35-45. doi:10.1016/j.exger.2015.02.008

14. Lima RM, de Oliveira RJ, Raposo R, Neri SGR, Gadelha AB. Stages of sarcopenia, bone mineral density, and the prevalence of osteoporosis in older women. Archives of osteoporosis. 2019;14:38. doi:10.1007/s11657-019-0591-4

15. Akpinar TS, Tayfur M, Tufan F, Sahinkaya T, Kose M, Ozsenel EB, et al. Uncomplicated diabetes does not accelerate age-related sarcopenia. The aging male : the official journal of the International Society for the Study of the Aging Male. 2014;17:205-10. doi:10.3109/13685538.2014.963040

16. Farinatti P, Paes L, Harris EA, Lopes GO, Borges JP. A Simple Model to Identify Risk of Sarcopenia and Physical Disability in HIV-Infected Patients. Journal of strength and conditioning research. 2017;31:2542-51. doi:10.1519/jsc.0000000000002070

17. Gadelha AB, Vainshelboim B, Ferreira AP, Neri SGR, Bottaro M, Lima RM. Stages of sarcopenia and the incidence of falls in older women: A prospective study. Arch Gerontol Geriatr. 2018;79:151-7. doi:10.1016/j.archger.2018.07.014

18. McGrath R, Hackney KJ, Ratamess NA, Vincent BM, Clark BC, Kraemer WJ. Absolute and Body Mass Index Normalized Handgrip Strength Percentiles by Gender, Ethnicity, and Hand Dominance in Americans. Advances in geriatric medicine and research. 2020;2:doi:10.20900/agmr20200005

19. Manini TM, Visser M, Won-Park S, Patel KV, Strotmeyer ES, Chen H, et al. Knee extension strength cutpoints for maintaining mobility. J Am Geriatr Soc. 2007;55:451-7. doi:10.1111/j.1532-5415.2007.01087.x 
medRxiv preprint doi: https://doi.org/10.1101/2021.07.28.21261230; this version posted July 31, 2021. The copyright holder for this preprint (which was not certified by peer review) is the author/funder, who has granted medRxiv a license to display the preprint in perpetuity.

20. Foley KT, Owings TM, Pavol MJ, Grabiner MD. Maximum grip strength is not related to bone mineral density of the proximal femur in older adults. Calcified Tissue International. 1999;64:291-4.

21. Maranhao Neto GA, Oliveira AJ, Pedreiro RC, Pereira-Junior PP, Machado S, Marques Neto S, et al. Normalizing handgrip strength in older adults: An allometric approach. Archives of Gerontology and Geriatrics. 2017;70:230-4. doi:10.1016/j.archger.2017.02.007

22. Pua Y-H. Allometric analysis of physical performance measures in older adults. Physical therapy. 2006;86:1263-70.

23. Abdalla PP, Dos Santos Carvalho A, Dos Santos AP, Venturini ACR, Alves TC, Mota $\mathrm{J}$, et al. Cut-off points of knee extension strength allometrically adjusted to identify sarcopenia risk in older adults: A cross-sectional study. Arch Gerontol Geriatr. 2020;89:104100. doi:10.1016/j.archger.2020.104100

24. Abdalla PP, Venturini ACR, Santos APD, Tasinafo M, Marini JAG, Alves TC, et al. Normalizing calf circumference to identify low skeletal muscle mass in older women: a crosssectional study. Nutr Hosp. 2021;doi:10.20960/nh.03572

25. DAVIES MJ, DALSKY GP. Normalizing strength for body size differences in older adults. Medicine \& Science in Sports \& Exercise. 1997;29:713-7.

26. Bouchard DR, Beliaeff S, Dionne IJ, Brochu M. Fat Mass But Not Fat-Free Mass Is Related to Physical Capacity in Well-Functioning Older Individuals: Nutrition as a Determinant of Successful Aging (NuAge) - The Quebec Longitudinal Study. The Journals of Gerontology: Series A. 2007;62:1382-8. doi:10.1093/gerona/62.12.1382

27. Broadwin J, Goodman-Gruen D, Slymen D. Ability of Fat and Fat-Free Mass Percentages to Predict Functional Disability in Older Men and Women. Journal of the American Geriatrics Society. 2001;49:1641-5. doi:https://doi.org/10.1111/j.15325415.2001.49273.x

28. $\quad$ Enright PL. The Six-Minute Walk Test. Respiratory Care. 2003;48:783-5. 29. Binder EF, Yarasheski KE, Steger-May K, Sinacore DR, Brown M, Schechtman KB, et al. Effects of progressive resistance training on body composition in frail older adults: results of a randomized, controlled trial. The Journals of Gerontology: Series A. 2005;60:1425-31.

30. Cruz-Jentoft AJ, Landi F, Schneider SM, Zuniga C, Arai H, Boirie Y, et al. Prevalence of and interventions for sarcopenia in ageing adults: a systematic review. Report of the International Sarcopenia Initiative (EWGSOP and IWGS). Age and ageing. 2014;43:748-59. doi:10.1093/ageing/afu115

31. Finney GR, Minagar A, Heilman KM. Assessment of Mental Status. Neurologic clinics. 2016;34:1-16. doi:10.1016/j.ncl.2015.08.001

32. Icaza MC, Albala C. Projeto SABE. Minimental State Examination (MMSE) del estudio de dementia en Chile: análisis estatístico Brasília: OPAS. 1999;1-18.

33. WHO Expert Consultation. Appropriate body-mass index for Asian populations and its implications for policy and intervention strategies. Lancet (London, England). 2004;363:157-63. doi:10.1016/s0140-6736(03)15268-3

34. Segal NA, Torner JC, Yang M, Curtis JR, Felson DT, Nevitt MC. Muscle mass is more strongly related to hip bone mineral density than is quadriceps strength or lower activity level in adults over age 50 year. Journal of Clinical Densitometry. 2008;11:503-10. doi:10.1016/j.jocd.2008.03.001

35. Bailey BJ, Briars GL. Estimating the surface area of the human body. Statistics in medicine. 1996;15:1325-32.

36. Jelliffe DB, JELLIFFE EP. The arm circumference as a public health index of proteincalorie malnutrition of early childhood. 20. Current conclusions. Journal of tropical pediatrics. 1969;15:253-60. 
medRxiv preprint doi: https://doi.org/10.1101/2021.07.28.21261230; this version posted July 31, 2021. The copyright holder for this preprint (which was not certified by peer review) is the author/funder, who has granted medRxiv a license to display the preprint in perpetuity.

37. Heymsfield SB, McManus C, Smith J, Stevens V, Nixon DW. Anthropometric measurement of muscle mass: revised equations for calculating bone-free arm muscle area. Am J Clin Nutr. 1982;36:680-90.

38. George LB, Bruce RB, Baltej SM, Haran TS, Michael FS. Nutritional and metabolic assessment of the hospitalized patient. Journal of Parenteral and Enteral Nutrition. 1977;1:1121. doi:10.1177/014860717700100101

39. Lean M, Han TS, Deurenberg P. Predicting body composition by densitometry from simple anthropometric measurements. The American journal of clinical nutrition. 1996;63:414.

40. Baumgartner RN, Koehler KM, Gallagher D, Romero L, Heymsfield SB, Ross RR, et al. Epidemiology of sarcopenia among the elderly in New Mexico. American journal of epidemiology. 1998;147:755-63.

41. Baumgartner RN, Heymsfield SB, Lichtman S, Wang J, Pierson RN, Jr. Body composition in elderly people: effect of criterion estimates on predictive equations. Am J Clin Nutr. 1991;53:1345-53. doi:10.1093/ajen/53.6.1345

42. LOHMAN TG, ROCHE AF, MARTORELLI R. Anthropometric standardization reference manual. Champaing human kinects 1988;1:

43. Morley JE, Abbatecola AM, Argiles JM, Baracos V, Bauer J, Bhasin S, et al. Sarcopenia with limited mobility: an international consensus. J Am Med Dir Assoc. 2011;12:403-9. doi:10.1016/j.jamda.2011.04.014

44. Massy-Westropp NM, Gill TK, Taylor AW, Bohannon RW, Hill CL. Hand Grip Strength: age and gender stratified normative data in a population-based study. BMC Research Notes. 2011;4:1-5.

45. Lourenco R, Perez-Zepeda M, Gutierrez-Robledo L, Rodriguez Manas L, GarciaGarcia F. Performance of the European Working Group on Sarcopenia in Older People algorithm in screening older adults for muscle mass assessment. Age and ageing.

2014;44:334-8. doi:10.1093/ageing/afu192

46. Alexandre S, Duarte YA, Santos JL, Wong R, Lebrao ML. Prevalence and associated factors of sarcopenia among elderly in Brazil: findings from the SABE study. The Journal of Nutrition, Health \& Aging. 2014;18:284-90. doi:10.1007/s12603-013-0413-0

47. Brzycki M. Strength testing-predicting a one-rep max from reps-to-fatigue. Journal of Physical Education, Recreation \& Dance. 1993;64:88-90.

48. Abdalla PP, Carvalho AdS, dos Santos AP, Venturini ACR, Alves TC, Mota J, et al. One-repetition submaximal protocol to measure knee extensor muscle strength among older adults with and without sarcopenia: a validation study. BMC Sports Science, Medicine and Rehabilitation. 2020;12:29. doi:10.1186/s13102-020-00178-9

49. Matsudo S, Araújo T, Matsudo V, Andrade D, Andrade E, Oliveira LC, et al. Questionário internacional De atividade física (ipaq): estupo De validade e reprodutibilidade No Brasil. Revista Brasileira de Atividade Física \& Saúde. 2012;6:5-18.

50. Myers R. Classical and modern regression with applications. Boston: PWS and Kent Publishing Company. Inc; 1990.

51. Mukaka MM. Statistics corner: A guide to appropriate use of correlation coefficient in medical research. Malawi Med J. 2012;24:69-71.

52. Schisterman EF, Perkins NJ, Liu A, Bondell H. Optimal cut-point and its corresponding Youden Index to discriminate individuals using pooled blood samples. Epidemiology. 2005;16:73-81.

53. Hosmer D, Lemeshow S. Applied logistic regression. 2 ed. Nova Jersey, EUA: John Wiley \& Sons; 2000.

54. Cawthon PM, Manini T, Patel SM, Newman A, Travison T, Kiel DP, et al. Putative Cut-Points in Sarcopenia Components and Incident Adverse Health Outcomes: An SDOC 
medRxiv preprint doi: https://doi.org/10.1101/2021.07.28.21261230; this version posted July 31, 2021. The copyright holder for this preprint (which was not certified by peer review) is the author/funder, who has granted medRxiv a license to display the preprint in perpetuity. It is made available under a CC-BY-NC-ND 4.0 International license.

Analysis. Journal of the American Geriatrics Society. 2020;68:1429-37. doi:https://doi.org/10.1111/jgs.16517

55. Alley DE, Shardell MD, Peters KW, McLean RR, Dam T-TL, Kenny AM, et al. Grip Strength Cutpoints for the Identification of Clinically Relevant Weakness. The Journals of Gerontology: Series A. 2014;69:559-66. doi:10.1093/gerona/glu011

56. Owings TM, Pavol MJ, Grabiner MD. Lower extremity muscle strength does not independently predict proximal femur bone mineral density in healthy older adults. Bone. 2002;30:515-20.

57. Ramírez-Vélez R, Sáez de Asteasu ML, Martínez-Velilla N, Zambom-Ferraresi F, García-Hermoso A, Izquierdo M. Handgrip Strength as a Complementary Test for Mobility Limitations Assessment in Acutely Hospitalized Oldest Old. Rejuvenation Res.

2021; doi:10.1089/rej.2020.2344

58. Jaric S. Role of body size in the relation between muscle strength and movement performance. Exercise and Sport Sciences Reviews. 2003;31:8-12. 
medRxiv preprint doi: https://doi.org/10.1101/2021.07.28.21261230; this version posted July 31,2021 . The copyright holder for this preprint (which was not certified by peer review) is the author/funder, who has granted medRxiv a license to display the preprint in perpetuity.

It is made available under a CC-BY-NC-ND 4.0 International license.

\section{TABELS}

Table 1. Allometric exponents $\left({ }^{b}\right)$ proposed in previous studies.

\begin{tabular}{|c|c|}
\hline Authors & Normalized muscle strength for body-size variable \\
\hline Jaric et al., (2003) [58] & General muscle strength/body mass ${ }^{\mathbf{0 . 6 7}}$ \\
\hline Foley et al., (1999) [20] & HGS/body mass ${ }^{\mathbf{0 . 4 0}}$ \\
\hline Pua (2006) [22] & HGS/body mass ${ }^{\mathbf{0 . 6 3}}$ \\
\hline \multirow{2}{*}{ Maranhão Neto et al., (2017) [21] } & HGS/body mass ${ }^{\mathbf{0 . 3 1}}$ \\
\hline & HGS/height ${ }^{1.84}$ \\
\hline \multirow{2}{*}{ Abdalla et al., 2020 [23] } & $1 \mathrm{RM}_{\text {knee extensors }} /$ body mass ${ }^{\mathbf{0 . 6 9}}$ \\
\hline & $1 \mathrm{RM}_{\text {knee extensors }} /$ body mass $^{\mathbf{0 . 9 6}}$ \\
\hline \multirow{3}{*}{ Davies and Dalsky (1997) [25] } & knee extension $\mathrm{PT}^{60 \% / s} /$ body mass ${ }^{\mathbf{0 . 6 7}}$ \\
\hline & knee extension $\mathrm{PT}^{60 \%} / \mathrm{s}$ body mass ${ }^{\mathbf{0 . 7 2}}$ \\
\hline & knee extension $\mathrm{PT}^{60 \%} / \mathrm{s} /$ body mass ${ }^{\mathbf{0 . 7 4}}$ \\
\hline Segal et al., (2008) [34] & knee extension $\mathrm{PT}^{60 \% / \mathrm{s}} /$ body mass $*$ height $^{0.97}$ \\
\hline
\end{tabular}

peak torque at $60 \%$ 
medRxiv preprint doi: https://doi.org/10.1101/2021.07.28.21261230; this version posted July 31, 2021. The copyright holder for this preprint (which was not certified by peer review) is the author/funder, who has granted medRxiv a license to display the preprint in perpetuity.

Table 2. Descriptive analysis and significant correlations of muscle strength with body-size variables in older men and women $(n=94)$.

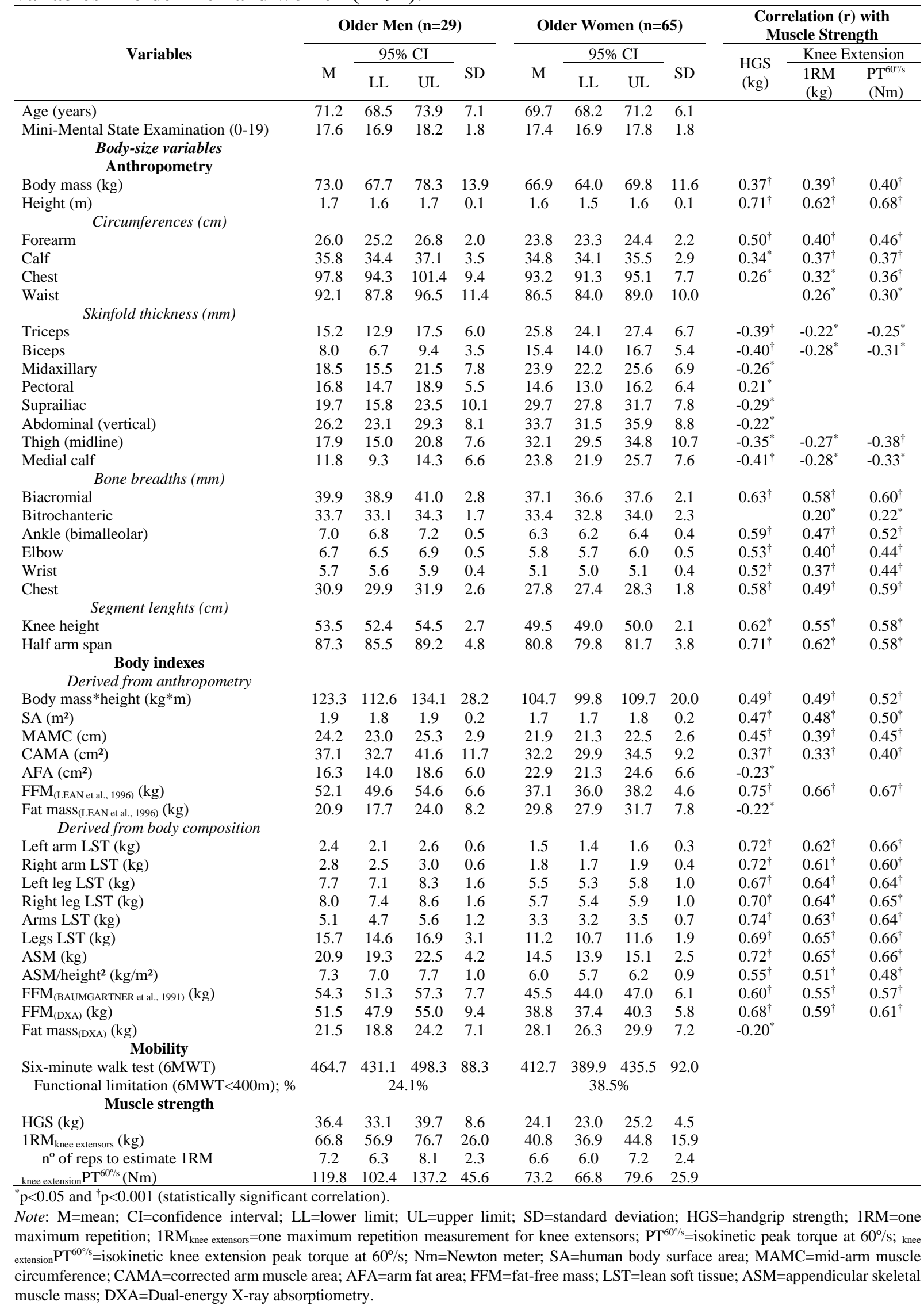


medRxiv preprint doi: https://doi.org/10.1101/2021.07.28.21261230; this version posted July 31, 2021. The copyright holder for this preprint (which was not certified by peer review) is the author/funder, who has granted medRxiv a license to display the preprint in perpetuity. It is made available under a CC-BY-NC-ND 4.0 International license.

Table 3. Adequate cut-off points (AUC $\geq 0.70$ simultaneously in both sexes and $\mathrm{r} \leq 0.30$ with body size) of handgrip strength (HGS), one maximum repetition measurement for knee extensors $\left(1 \mathrm{RM}_{\text {knee extensors }}\right)$ and isokinetic knee extension peak torque at $60 \% \mathrm{~s}\left(\mathrm{knee}\right.$ extension $\left.\mathrm{PT}^{60 \%}\right)$ to identify muscle weakness.

\begin{tabular}{|c|c|c|c|c|c|c|c|c|c|}
\hline \multirow[b]{2}{*}{ Variable } & \multirow[b]{2}{*}{ Unit } & \multicolumn{4}{|c|}{$\operatorname{Men}(n=29)$} & \multicolumn{4}{|c|}{ Women $(n=65)$} \\
\hline & & AUC & $\begin{array}{c}\text { Cut-off point } \\
(\leq)\end{array}$ & $\begin{array}{c}\text { Sens } \\
(\%)\end{array}$ & $\begin{array}{l}\text { Spe } \\
(\%)\end{array}$ & AUC & $\begin{array}{c}\text { Cut-off point } \\
(\leq)\end{array}$ & $\begin{array}{c}\text { Sens } \\
(\%)\end{array}$ & $\begin{array}{l}\text { Spe } \\
(\%)\end{array}$ \\
\hline \multicolumn{10}{|l|}{$H G S(k g)$} \\
\hline /forearm circumference & $(\mathrm{cm})$ & $0.74^{*}$ & 1.33 & 86 & 59 & $0.70^{*}$ & 1.04 & 84 & 58 \\
\hline \multicolumn{10}{|l|}{$1 R M_{\text {knee extensors }}(\mathrm{kg})$} \\
\hline /body mass & $(\mathrm{kg})$ & $0.77^{*}$ & 0.85 & 86 & 68 & $0.76^{\dagger}$ & 0.54 & 68 & 78 \\
\hline /forearm circumference & & $0.75^{*}$ & 2.16 & 86 & 77 & $0.70^{*}$ & 1.38 & 60 & 75 \\
\hline /calf circumference & & $0.74^{*}$ & 1.65 & 86 & 77 & $0.70^{*}$ & 1.06 & 72 & 68 \\
\hline /chest circumference & (cm) & $0.76^{*}$ & 0.64 & 86 & 73 & $0.71^{*}$ & 0.4 & 72 & 65 \\
\hline /waist circumference & & $0.78^{*}$ & 0.73 & 100 & 59 & $0.72^{*}$ & 0.37 & 60 & 80 \\
\hline /triceps skinfold & & $0.81^{\dagger}$ & 4.22 & 86 & 68 & $0.70^{*}$ & 1.40 & 60 & 75 \\
\hline /bitrochanteric breadth & & $0.73^{*}$ & 1.72 & 86 & 77 & $0.70^{*}$ & 1.16 & 76 & 60 \\
\hline /bimalleolar breadth & $(\mathrm{mm})$ & $0.73^{*}$ & 8.77 & 86 & 77 & $0.70^{*}$ & 5.77 & 76 & 65 \\
\hline /elbow breadth & & $0.73^{*}$ & 9.36 & 86 & 73 & $0.70^{*}$ & 6.57 & 76 & 63 \\
\hline /SA & $\left(\mathrm{m}^{2}\right)$ & $0.75^{*}$ & 31.6 & 86 & 77 & $0.72^{*}$ & 21.2 & 72 & 68 \\
\hline /MAMC & $(\mathrm{cm})$ & $0.77^{*}$ & 2.42 & 86 & 73 & $0.72^{*}$ & 1.54 & 64 & 75 \\
\hline /CAMA & $\left(\mathrm{cm}^{2}\right)$ & $0.71^{*}$ & 2.03 & 100 & 41 & $0.73^{*}$ & 0.90 & 52 & 93 \\
\hline$/ \mathrm{FFM}_{\text {(LEAN et al., } 1996)}$ & $(\mathrm{kg})$ & $0.76^{*}$ & 1.11 & 86 & 77 & $0.72^{*}$ & 1.00 & 72 & 68 \\
\hline /FFM (BAUMGARTNER et al., 1991) & $(\mathrm{kg})$ & $0.78^{*}$ & 1.13 & 83 & 73 & $0.75^{\dagger}$ & 0.83 & 76 & 64 \\
\hline /body mass ${ }^{0.44}$ & & $0.75^{*}$ & 9.04 & 86 & 77 & $0.71^{*}$ & 6.03 & 76 & 63 \\
\hline /body mass ${ }^{0,67}$ (JARIC, 2003) & & $0.78^{*}$ & 3.40 & 86 & 77 & $0.73^{\dagger}$ & 2.28 & 76 & 65 \\
\hline /body mass ${ }^{0.96 \text { (ABDALLA et al., 2020) }}$ & (kg) & $0.77^{*}$ & 1.00 & 86 & 68 & $0.75^{\dagger}$ & 0.45 & 44 & 100 \\
\hline /body mass ${ }^{0.69 \text { (ABDALLA et al., 2020) }}$ & & $0.78^{*}$ & 3.06 & 86 & 77 & $0.73^{*}$ & 1.48 & 44 & 98 \\
\hline /calf circumference ${ }^{1.10}$ & $(\mathrm{~cm})$ & $0.75^{*}$ & 1.14 & 86 & 77 & $0.71^{*}$ & 0.70 & 68 & 73 \\
\hline /bimalleolar breadth ${ }^{1.20}$ & $(\mathrm{~mm})$ & $0.71^{*}$ & 6.01 & 86 & 68 & $0.70^{*}$ & 3.93 & 76 & 65 \\
\hline$/(\text { body mass *height })^{0.48}$ & $(\mathrm{~kg} * \mathrm{~m})$ & $0.75^{*}$ & 5.83 & 86 & 77 & $0.72^{*}$ & 4.06 & 76 & 65 \\
\hline$/ \mathrm{SA}^{0.93}$ & $\left(\mathrm{~m}^{2}\right)$ & $0.75^{*}$ & 33 & 86 & 77 & $0.72^{*}$ & 22.7 & 76 & 65 \\
\hline$/ \mathrm{FFM}^{0.88}$ (LEAN et al., 1996) & & $0.75^{*}$ & 1.77 & 86 & 77 & $0.71^{*}$ & 1.53 & 76 & 65 \\
\hline$/ \mathrm{FFM}^{0.67}$ (BAUMGARTNER et al., 1991) & (kg) & $0.76^{*}$ & 3.94 & 83 & 77 & $0.72^{*}$ & 2.91 & 76 & 64 \\
\hline \multicolumn{10}{|l|}{ knee extensors $P T^{60 \% / s}(\mathrm{Nm})$} \\
\hline /height & (m) & $0.93^{\dagger}$ & 54.1 & 86 & 95 & $0.74^{\dagger}$ & 44.1 & 64 & 75 \\
\hline /knee height & $(\mathrm{cm})$ & $0.94^{\dagger}$ & 1.83 & 100 & 86 & $0.75^{\dagger}$ & 1.44 & 68 & 73 \\
\hline /SA & $\left(\mathrm{m}^{2}\right)$ & $0.94^{\dagger}$ & 56.9 & 100 & 82 & $0.81^{\dagger}$ & 36.3 & 64 & 88 \\
\hline$/$ FFM $_{\text {(LEAN et al., } 1996)}$ & $(\mathrm{kg})$ & $0.93^{\dagger}$ & 1.79 & 86 & 95 & $0.81^{\dagger}$ & 1.84 & 76 & 80 \\
\hline /left leg LST & & $0.82^{\dagger}$ & 0.015 & 100 & 55 & $0.76^{\dagger}$ & 0.012 & 68 & 80 \\
\hline /right leg LST & (g) & $0.77^{*}$ & 0.015 & 100 & 50 & $0.78^{\dagger}$ & 0.013 & 76 & 78 \\
\hline /legs LST & & $0.81^{*}$ & 0.0063 & 71 & 82 & $0.77^{\dagger}$ & 0.0065 & 76 & 78 \\
\hline /ASM & & $0.81^{*}$ & 4.66 & 71 & 86 & $0.77^{\dagger}$ & 5.01 & 76 & 78 \\
\hline FFM $_{\text {(BAUMGARTNER et al., 1991) }}$ & $(\mathrm{kg})$ & $0.93^{\dagger}$ & 1.63 & 83 & 95 & $0.85^{\dagger}$ & 1.60 & 88 & 77 \\
\hline$/ \mathrm{FFM}_{(\mathrm{DXA})}$ & & $0.84^{\dagger}$ & 2.08 & 100 & 59 & $0.79^{\dagger}$ & 1.89 & 76 & 75 \\
\hline /body mass ${ }^{0.67}$ (DAVIES; DALSKY, 1997) & & $0.93^{\dagger}$ & 5.06 & 86 & 95 & $0.82^{\dagger}$ & 3.71 & 68 & 88 \\
\hline /body mass ${ }^{0.72(\text { DAVIES; DALSKY, 1997) }}$ & & $0.94^{\dagger}$ & 4.1 & 86 & 95 & $0.82^{\dagger}$ & 3.14 & 72 & 85 \\
\hline /body mass ${ }^{0.74 \text { (DAVIES; DALSKY, 1997) }}$ & (kg) & $0.93^{\dagger}$ & 3.77 & 86 & 95 & $0.82^{\dagger}$ & 2.87 & 72 & 85 \\
\hline /body mass ${ }^{0.67 \text { (JARIC, 2003) }}$ & & $0.93^{\dagger}$ & 5.06 & 86 & 95 & $0.82^{\dagger}$ & 3.71 & 68 & 88 \\
\hline height $^{3.27}$ & (m) & $0.86^{\dagger}$ & 19.2 & 100 & 77 & $0.74^{\dagger}$ & 17.4 & 72 & 65 \\
\hline /knee height $^{1.82}$ & & $0.90^{\dagger}$ & 0.068 & 100 & 86 & $0.74^{\dagger}$ & 0.053 & 64 & 75 \\
\hline /half arm span ${ }^{1.62}$ & $(\mathrm{~cm})$ & $0.88^{\dagger}$ & 0.076 & 100 & 73 & $0.75^{\dagger}$ & 0.066 & 84 & 58 \\
\hline /biacromial breadth ${ }^{2.15}$ & & $0.82^{\dagger}$ & 0.038 & 86 & 68 & $0.77^{\dagger}$ & 0.03 & 76 & 73 \\
\hline /bimalleolar breadth ${ }^{1.54}$ & $(\mathrm{~mm})$ & $0.81^{*}$ & 5.04 & 86 & 73 & $0.80^{\dagger}$ & 4.64 & 92 & 60 \\
\hline$/(\text { body mass *height })^{0.43}$ & $(\mathrm{~kg} * \mathrm{~m})$ & $0.94^{\dagger}$ & 13 & 100 & 82 & $0.80^{\dagger}$ & 10.5 & 84 & 65 \\
\hline$/ \mathrm{SA}^{0.83}$ & $\left(\mathrm{~m}^{2}\right)$ & $0.94^{\dagger}$ & 53.8 & 86 & 95 & $0.80^{\dagger}$ & 50 & 84 & 65 \\
\hline /left leg $\mathrm{LST}^{0.43}$ & & $0.92^{\dagger}$ & 2.26 & 100 & 77 & $0.77^{\dagger}$ & 1.83 & 76 & 70 \\
\hline /right leg $\mathrm{LST}^{0.48}$ & (g) & $0.90^{\dagger}$ & 1.39 & 100 & 73 & $0.78^{\dagger}$ & 1.16 & 80 & 70 \\
\hline /legs LST ${ }^{0.47}$ & & $0.92^{\dagger}$ & 1.07 & 100 & 73 & $0.77^{\dagger}$ & 0.87 & 76 & 70 \\
\hline
\end{tabular}

${ }^{*} \mathrm{p}<0.05$ and ${ }^{\dagger} \mathrm{p}<0.001$ (statistically significant AUC)

Dependent variable (primary outcome): functional limitation $(6 \mathrm{MWT}<400 \mathrm{~m})$

Note: $\mathrm{AUC}=$ area under the curve; $p=$ significance; Sens=sensibility; Spe=specificity; $\mathrm{SA}=$ human body surface area; $\mathrm{MAMC}=$ mid-arm muscle circumference; $\mathrm{CAMA}=$ corrected arm muscle area; FFM=Fat-free mass; LST=lean soft tissue; ASM=appendicular skeletal muscle mass;

DXA=Dual-energy X-ray absorptiometry; 6MWT=six-minute walk test. 
medRxiv preprint doi: https://doi.org/10.1101/2021.07.28.21261230; this version posted July 31,2021 . The copyright holder for this preprint (which was not certified by peer review) is the author/funder, who has granted medRxiv a license to display the preprint in perpetuity.

\section{FIGURES}

Figure 1. Study phases and data from older adults included, excluded, analyzed and procedure flow.

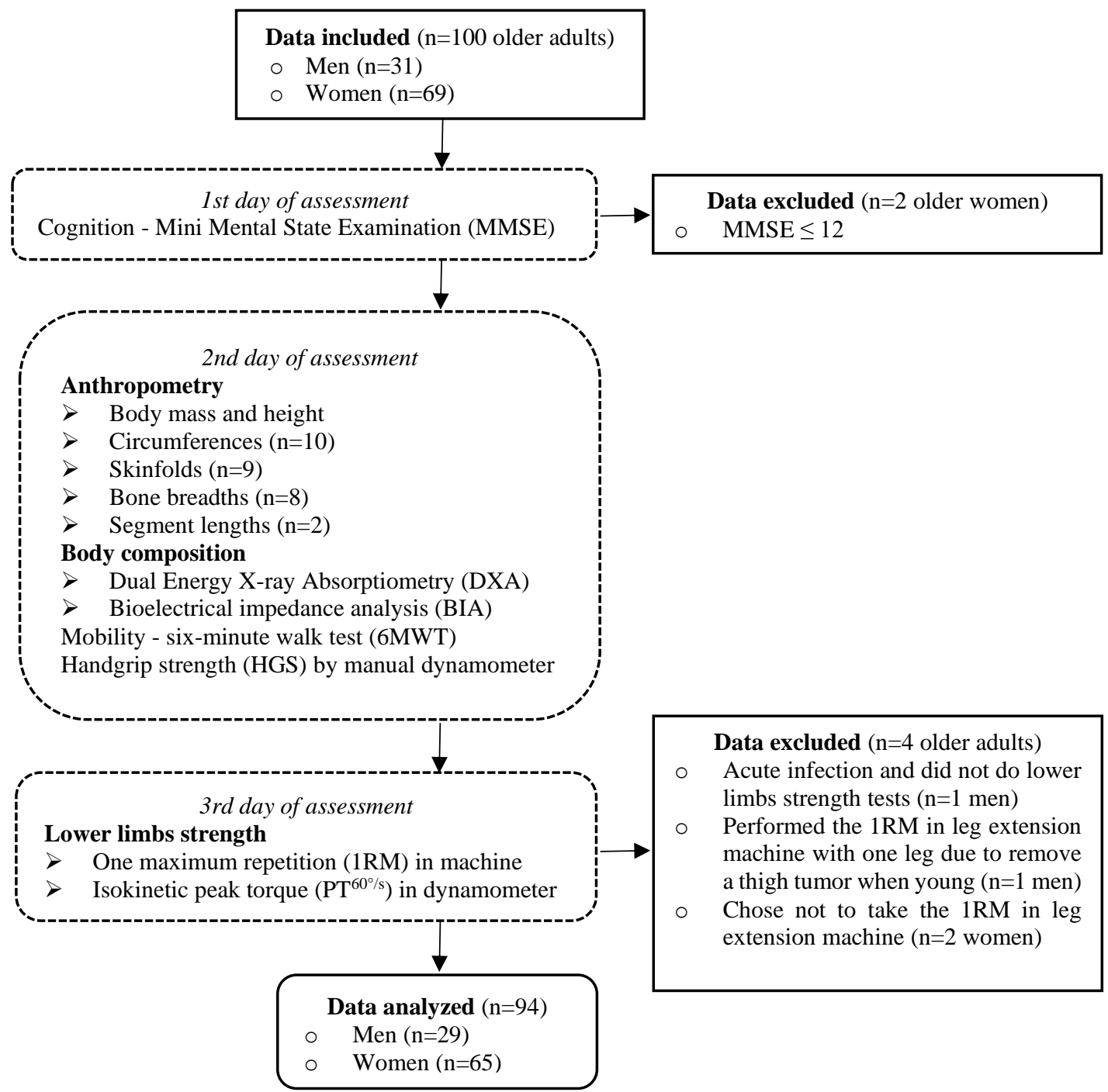


Figure 2. Accuracy comparison between non-normalized, ratio standard and allometric scaling of muscle weakness cut-off points of HGS and lower limbs strength ( $1 \mathrm{RM}_{\text {knee extensors }}$ and knee extension $\left.\mathrm{PT}^{60 \%}\right)$ in older men (letters $\left.\mathrm{a}, \mathrm{b}, \mathrm{c}\right)$ and older women (letters d, e, f).

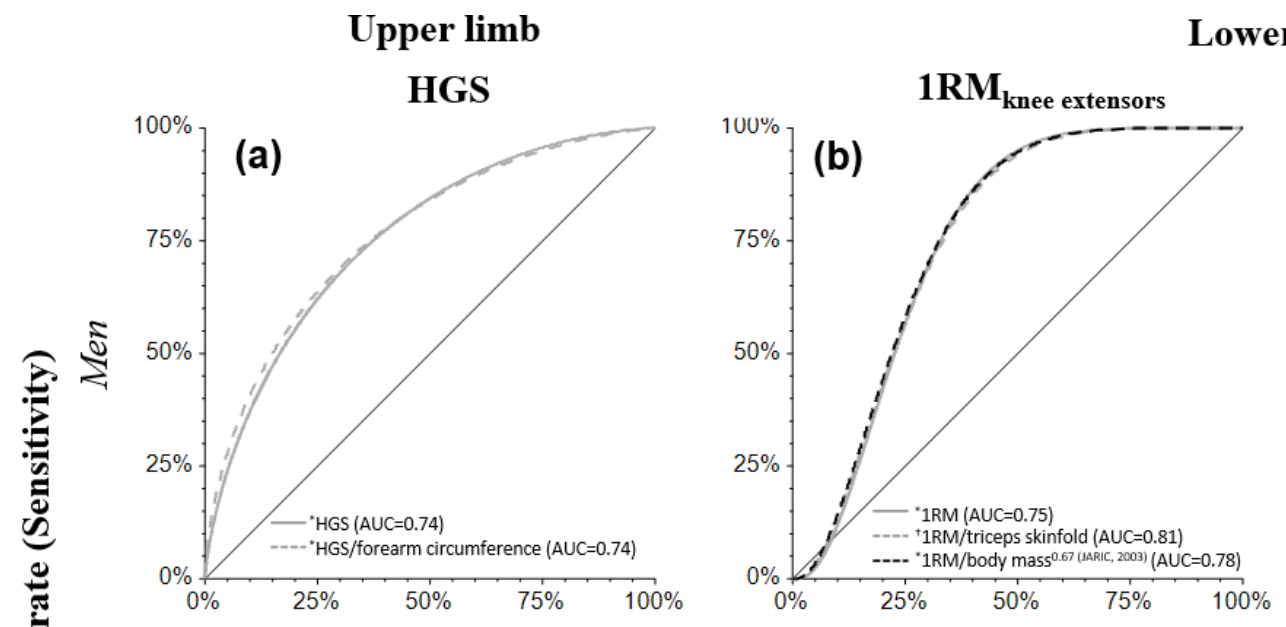

\section{Lower limbs}
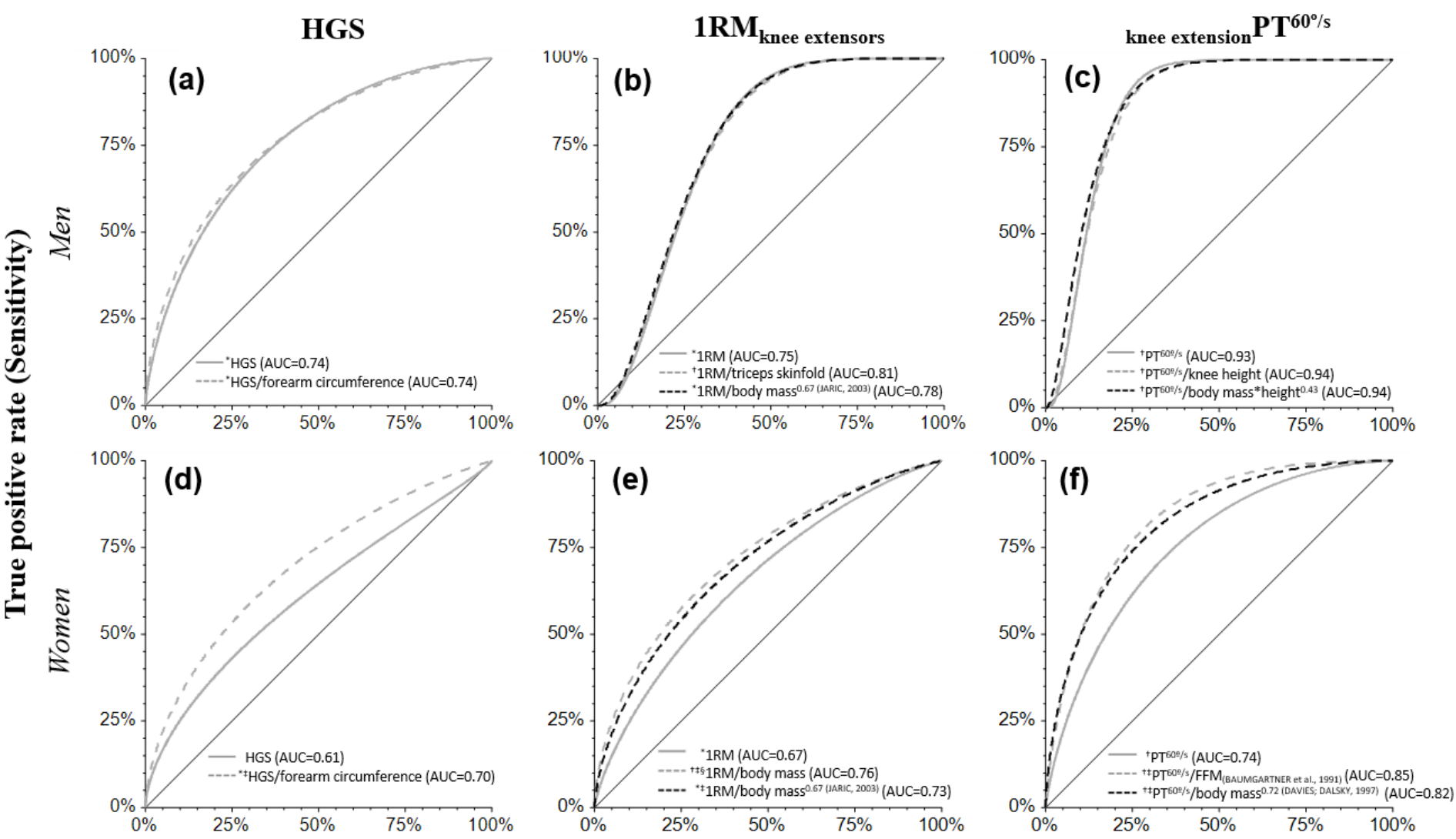

False positive rate (1-Specificity)

" $p<0.05$ and ${ }^{\dagger} \mathrm{p}<0.001$ (statistically significant AUC).

p $<0.05$ (greater than the AUC of non-normalized muscle strength).

$s_{\mathrm{p}}<0.05$ (greater than the AUC of the allometric scaling).

Dependent variable (primary outcome): functional limitation $(6 \mathrm{MWT}<400 \mathrm{~m})$

Note: $\mathrm{HGS}=$ handgrip strength; $1 \mathrm{RM}_{\text {knee extensors }}=$ one maximum repetition measurement for knee extensors; knee extension $^{\mathrm{PT}} \mathrm{T}^{60 \%}=\mathrm{s}=\mathrm{sokinetic}$ knee extension peak torque at $60^{\circ} / \mathrm{s} ; 6 \mathrm{MWT}=\mathrm{six}-\mathrm{minute}$ walk tes 
medRxiv preprint doi: https://doi.org/10.1101/2021.07.28.21261230; this version posted July 31,2021 . The copyright holder for this preprint (which was not certified by peer review) is the author/funder, who has granted medRxiv a license to display the preprint in perpetuity.

It is made available under a CC-BY-NC-ND 4.0 International license.

\section{SUPPLEMENTS}

SUPPLEMENT A - Body size variables $(n=49)$ to normalize muscle strength.

\begin{tabular}{lll}
\hline & \multicolumn{1}{c}{ Anthropometry } \\
\hline $\begin{array}{l}\text { Body mass }(\mathrm{kg}) \\
\text { Height }(\mathrm{m})\end{array}$ & \multicolumn{1}{c}{ Skinfold site $(\mathrm{mm})$} & \\
\multicolumn{1}{c}{ Circumferences $(\mathrm{cm})$} & \multicolumn{1}{c}{ Bone breadths $(\mathrm{mm})$} \\
Arm & Subescapular & Biacromial \\
Forearm & Triceps & Biiliac \\
Midthigh & Biceps & Bitrochanteric \\
Calf & Midaxillary & Ankle (bimalleolar) \\
Chest & Pectoral (chest) & Elbow \\
Waist & Suprailiac & Wrist \\
Abdomen & Abdominal (vertical) & Knee \\
Buttocks (hip) & Thigh (midline) & Chest \\
& Medial calf & \\
Segment lenghts $(\mathrm{cm})$ & & \\
Knee height & & \\
Half arm span & & \\
& &
\end{tabular}
Derived from anthropometry

Body mass*height $(\mathrm{kg} * \mathrm{~m})$

$\mathrm{SA}\left(\mathrm{m}^{2}\right)$

MAMC $(\mathrm{cm})$

CAMA $\left(\mathrm{cm}^{2}\right)$

AFA $\left(\mathrm{cm}^{2}\right)$

FFM $_{\text {(LEAN et al., 1996) }}(\mathrm{kg})$

Fat mass (LEAN et al., 1996) $(\mathrm{kg})$
BMI $\left(\mathrm{kg} / \mathrm{m}^{2}\right)$

\section{Body indexes}

Left arm LST (g)

Derived from body composition

Right arm LST (g)

Left leg LST $(\mathrm{g})$

Right leg LST (g)

Arms LST (g)

Legs LST (g)

$\operatorname{ASM}(\mathrm{kg})$

ASM/height ${ }^{2}\left(\mathrm{~kg} / \mathrm{m}^{2}\right)$

FFM $_{\text {(BAUMGARTNER et al., 1991) }}(\mathrm{kg})$

$\mathrm{FFM}_{(\mathrm{DXA})}(\mathrm{kg})$

Fat mass(BAUMGARTNER et al., 1991) $(\mathrm{kg})$

Fat mass $(\mathrm{DXA})(\mathrm{kg})$

Note: $\mathrm{BMI}=$ body mass index; $\mathrm{SA}=$ surface area of human body; $\mathrm{MAMC}=$ mid-arm muscle circumference; $\mathrm{CAMA}=$ corrected arm muscle area; $\mathrm{AFA}=$ arm fat area; $\mathrm{FFM}=$ fat-free mass; $\mathrm{LST}=$ lean soft tissue; $\mathrm{ASM}=$ appendicular skeletal muscle mass.

Instruments utilized for measures: body mass (Filizola ${ }^{\circledR}$ digital scale, model Personal, Brazil), height (Sanny® wall-mounted aluminum stadiometer, Professional model ES2020, Brazil), circunferences (Sanny ${ }^{\circledR}$ inelastic and inextensible measuring tape, Brazil), skinfolds (Lange scientific skinfold caliper, Cambridge Scientific Instruments, Cambridge, Maryland), bone breadths (Sanny® anthropometer and small sliding caliper, Brazil), and segment lengths (Sanny® segmometer, Brazil), LST components, ASM and FFM $_{(\mathrm{DXA})}$ (Dual Energy X-ray Absorptiometry-DXA, Hologic ${ }^{\circledR}$, model QDR4500W, software version 11.2, Bedford, MA), FFM (BAUMGARTNER et al., 1991) (Bioimpedance Imp DF50 Body Composition Analysis, ImpediMed®, Brisbane, Queensland, Australia). 
medRxiv preprint doi: https://doi.org/10.1101/2021.07.28.21261230; this version posted July 31, 2021. The copyright holder for this preprint (which was not certified by peer review) is the author/funder, who has granted medRxiv a license to display the preprint in perpetuity.

SUPPLEMENT B - Linear regressions to obtain allometric exponents for handgrip strength (HGS), one maximum repetition measurement for knee extensors $\left(1 \mathrm{RM}_{\mathrm{knee}}\right.$ extensors $)$ and isokinetic knee extension peak torque at $60 \% \mathrm{~s}\left(\mathrm{knee}\right.$ extension $\left.\mathrm{PT}^{60 \%} \mathrm{~s}\right)$ in older men and women $(n=94)$.

\begin{tabular}{|c|c|c|c|c|c|c|c|}
\hline \multirow{2}{*}{$\begin{array}{c}\text { Linear } \\
\text { regression }\end{array}$} & \multirow{2}{*}{ Independent variables } & \multicolumn{2}{|c|}{ Regression coefficients } & \multicolumn{2}{|c|}{$95 \%$ CI of $\beta$} & \multirow{2}{*}{ VIF } & \multirow{2}{*}{$\begin{array}{c}\text { Adjusted } \\
\mathbf{R}^{2}\end{array}$} \\
\hline & & $\beta$ & Standard error & $\mathbf{L L}$ & UL & & \\
\hline \multicolumn{8}{|c|}{ Dependent variable: $\mathrm{HGS}$ (ln $\mathrm{kg})$} \\
\hline \multirow{5}{*}{1} & Constant & $3.040^{\dagger}$ & 0.669 & 1.711 & 4.368 & & \multirow{5}{*}{0.53} \\
\hline & Sex & $0.407^{\dagger}$ & 0.046 & 0.315 & 0.499 & 1.129 & \\
\hline & Age & $-0.012^{*}$ & 0.003 & -0.019 & -0.005 & 1.181 & \\
\hline & Physical activity level & 0.061 & 0.144 & -0.225 & 0.347 & 1.059 & \\
\hline & ln body mass (kg) & 0.219 & 0.122 & -0.024 & 0.462 & 1.218 & \\
\hline \multirow{5}{*}{2} & Constant & $3.069^{\dagger}$ & 0.380 & 2.314 & 3.823 & & \multirow{5}{*}{0.58} \\
\hline & Sex & $0.282^{\dagger}$ & 0.057 & 0.169 & 0.395 & 1.919 & \\
\hline & Age & $-0.010^{*}$ & 0.003 & -0.017 & -0.004 & 1.143 & \\
\hline & Physical activity level & -0.009 & 0.136 & -0.279 & 0.262 & 1.068 & \\
\hline & ln height $(\mathrm{m})$ & $1.875^{\dagger}$ & 0.491 & 0.899 & 2.85 & 1.891 & \\
\hline \multirow{5}{*}{3} & Constant & $2.393^{*}$ & 0.877 & 0.651 & 4.135 & & \multirow{5}{*}{0.54} \\
\hline & Sex & $0.381^{\dagger}$ & 0.050 & 0.281 & 0.480 & 1.334 & \\
\hline & Age & $-0.012^{\dagger}$ & 0.003 & -0.019 & -0.006 & 1.128 & \\
\hline & Physical activity level & 0.044 & 0.143 & -0.239 & 0.328 & 1.056 & \\
\hline & ln forearm circumference $(\mathrm{cm})$ & $0.501^{*}$ & 0.240 & 0.023 & 0.978 & 1.349 & \\
\hline \multirow{5}{*}{4} & Constant & 1.992 & 1.002 & $<0.001$ & 3.984 & & \multirow{5}{*}{0.54} \\
\hline & Sex & $0.413^{\dagger}$ & 0.045 & 0.323 & 0.502 & 1.084 & \\
\hline & Age & $-0.012^{*}$ & 0.003 & -0.018 & -0.005 & 1.186 & \\
\hline & Physical activity level & 0.084 & 0.143 & -0.200 & 0.368 & 1.071 & \\
\hline & ln calf circumference $(\mathrm{cm})$ & $0.536^{*}$ & 0.242 & 0.056 & 1.017 & 1.195 & \\
\hline & Constant & $3.728^{*}$ & 1.227 & 1.289 & 6.167 & & \\
\hline & Sex & $0.424^{\dagger}$ & 0.047 & 0.329 & 0.518 & 1.143 & \\
\hline 5 & Age & $-0.014^{\dagger}$ & 0.003 & -0.021 & -0.007 & 1.110 & 0.52 \\
\hline & Physical activity level & 0.048 & 0.146 & -0.242 & 0.338 & 1.057 & \\
\hline & ln chest circumference $(\mathrm{cm})$ & 0.084 & 0.248 & -0.409 & 0.576 & 1.162 & \\
\hline & Constant & -1.116 & 1.815 & -4.722 & 2.489 & & \\
\hline & Sex & $0.323^{\dagger}$ & 0.057 & 0.210 & 0.435 & 1.779 & \\
\hline 6 & Age & $-0.013^{\dagger}$ & 0.003 & -0.019 & -0.006 & 1.061 & 0.56 \\
\hline & Physical activity level & 0.02 & 0.140 & -0.258 & 0.297 & 1.061 & \\
\hline & ln knee height $(\mathrm{cm})$ & $1.318^{*}$ & 0.451 & 0.422 & 2.213 & 1.708 & \\
\hline & Constant & $-4.217^{*}$ & 1.867 & -7.927 & -0.507 & & \\
\hline & Sex & $0.283^{\dagger}$ & 0.052 & 0.179 & 0.387 & 1.705 & \\
\hline 7 & Age & $-0.010^{*}$ & 0.003 & -0.016 & -0.003 & 1.161 & 0.61 \\
\hline & Physical activity level & 0.086 & 0.132 & -0.176 & 0.349 & 1.061 & \\
\hline & ln half arm span $(\mathrm{cm})$ & $1.813^{\dagger}$ & 0.402 & 1.015 & 2.611 & 1.752 & \\
\hline & Constant & $2.942^{\dagger}$ & 0.659 & 1.633 & 4.251 & & \\
\hline & Sex & $1.070^{*}$ & 0.298 & 0.478 & 1.663 & 47.067 & \\
\hline & Age & $-0.011^{*}$ & 0.004 & -0.018 & -0.004 & 1.279 & \\
\hline 8 & Physical activity level & $0.647^{*}$ & 0.308 & 0.035 & 1.258 & 4.896 & 0.54 \\
\hline & ln triceps skinfold (mm) & 0.114 & 0.079 & -0.043 & 0.270 & 3.147 & \\
\hline & Interaction & $-0.003^{*}$ & 0.001 & -0.006 & $<0.001$ & 36.925 & \\
\hline & Constant & $4.213^{\dagger}$ & 0.343 & 3.530 & 4.895 & & \\
\hline & Sex & $0.413^{\dagger}$ & 0.059 & 0.296 & 0.53 & 1.752 & \\
\hline 9 & Age & $-0.015^{\dagger}$ & 0.003 & -0.021 & -0.008 & 1.041 & 0.52 \\
\hline & Physical activity level & 0.037 & 0.148 & -0.257 & 0.331 & 1.086 & \\
\hline & ln biceps skinfold (mm) & -0.021 & 0.053 & -0.126 & 0.083 & 1.706 & \\
\hline & Constant & $4.160^{\dagger}$ & 0.343 & 3.478 & 4.843 & & \\
\hline & Sex & $0.425^{\dagger}$ & 0.049 & 0.329 & 0.522 & 1.197 & \\
\hline 10 & Age & $-0.015^{\dagger}$ & 0.003 & -0.021 & -0.008 & 1.035 & 0.52 \\
\hline & Physical activity level & 0.044 & 0.147 & -0.247 & 0.336 & 1.067 & \\
\hline & ln midaxillary skinfold (mm) & -0.007 & 0.050 & -0.106 & 0.091 & 1.158 & \\
\hline & Constant & $2.809^{\dagger}$ & 0.523 & 1.769 & 3.848 & & \\
\hline & Sex & $1.055^{\dagger}$ & 0.256 & 0.546 & 1.564 & 36.193 & \\
\hline & Age & $-0.011^{*}$ & 0.003 & -0.018 & -0.005 & 1.154 & \\
\hline 11 & Physical activity level & $0.769^{*}$ & 0.307 & 0.160 & 1.378 & 5.064 & 0.56 \\
\hline & ln pectoral skinfold (mm) & $0.141^{*}$ & 0.055 & 0.032 & 0.249 & 1.384 & \\
\hline & $\begin{array}{l}\text { Interaction } \\
\text { S }\end{array}$ & $-0.003^{*}$ & 0.001 & $\begin{array}{l}0.052 \\
-0.006\end{array}$ & $\begin{array}{r}0.249 \\
-0.001\end{array}$ & $\begin{array}{l}1.504 \\
36.287\end{array}$ & \\
\hline
\end{tabular}


medRxiv preprint doi: https://doi.org/10.1101/2021.07.28.21261230; this version posted July 31, 2021. The copyright holder for this preprint (which was not certified by peer review) is the author/funder, who has granted medRxiv a license to display the preprint in perpetuity. It is made available under a CC-BY-NC-ND 4.0 International license.

\begin{tabular}{|c|c|c|c|c|c|c|c|}
\hline \multirow{2}{*}{$\begin{array}{c}\text { Linear } \\
\text { regression }\end{array}$} & \multirow{2}{*}{ Independent variables } & \multicolumn{2}{|c|}{ Regression coefficients } & \multicolumn{2}{|c|}{$95 \%$ CI of $\beta$} & \multirow{2}{*}{ VIF } & \multirow{2}{*}{$\begin{array}{c}\text { Adjusted } \\
\mathbf{R}^{2}\end{array}$} \\
\hline & & $\beta$ & Standard error & $\mathbf{L L}$ & $\mathbf{U L}$ & & \\
\hline \multirow{5}{*}{12} & Constant & $4.098^{\dagger}$ & 0.370 & 3.363 & 4.833 & & \multirow{5}{*}{0.52} \\
\hline & Sex & $0.431^{\dagger}$ & 0.053 & 0.327 & 0.536 & 1.412 & \\
\hline & Age & $-0.014^{\dagger}$ & 0.003 & -0.021 & -0.008 & 1.085 & \\
\hline & Physical activity level & 0.049 & 0.147 & -0.243 & 0.341 & 1.069 & \\
\hline & In suprailiac skinfold (mm) & 0.007 & 0.050 & -0.093 & 0.106 & 1.432 & \\
\hline \multirow{6}{*}{13} & Constant & $2.458^{*}$ & 0.682 & 1.101 & 3.814 & & \multirow{6}{*}{0.55} \\
\hline & Sex & $1.253^{\dagger}$ & 0.312 & 0.632 & 1.874 & 53.064 & \\
\hline & Age & $-0.010^{*}$ & 0.004 & -0.017 & -0.003 & 1.302 & \\
\hline & Physical activity level & $0.891^{*}$ & 0.346 & 0.203 & 1.578 & 6.352 & \\
\hline & ln abdominal skinfold (mm) & $0.148^{*}$ & 0.071 & 0.008 & 0.289 & 1.86 & \\
\hline & Interaction & $-0.004^{*}$ & 0.001 & -0.006 & -0.001 & 47.986 & \\
\hline \multirow{6}{*}{14} & Constant & $2.951^{\dagger}$ & 0.619 & 1.720 & 4.181 & & \multirow{6}{*}{0.54} \\
\hline & Sex & $1.059^{\dagger}$ & 0.284 & 0.495 & 1.624 & 42.851 & \\
\hline & Age & $-0.011^{*}$ & 0.004 & -0.018 & -0.004 & 1.246 & \\
\hline & Physical activity level & $0.676^{*}$ & 0.314 & 0.052 & 1.300 & 5.107 & \\
\hline & ln thigh skinfold (mm) & 0.092 & 0.064 & -0.034 & 0.219 & 2.412 & \\
\hline & Interaction & $-0.003^{*}$ & 0.001 & -0.006 & $<0.001$ & 35.064 & \\
\hline & Constant & $4.256^{\dagger}$ & 0.335 & 3.590 & 4.921 & & \\
\hline & Sex & $0.403^{\dagger}$ & 0.058 & 0.287 & 0.519 & 1.737 & \\
\hline 15 & Age & $-0.015^{\dagger}$ & 0.003 & -0.021 & -0.008 & 1.041 & 0.52 \\
\hline & Physical activity level & 0.041 & 0.146 & -0.249 & 0.331 & 1.06 & \\
\hline & ln medial calf skinfold (mm) & -0.031 & 0.046 & -0.121 & 0.060 & 1.711 & \\
\hline & Constant & -0.060 & 1.519 & -3.077 & 2.958 & & \\
\hline & Sex & $0.348^{\dagger}$ & 0.052 & 0.244 & 0.452 & 1.506 & \\
\hline 16 & Age & $-0.010^{*}$ & 0.004 & -0.017 & -0.003 & 1.295 & 0.56 \\
\hline & Physical activity level & 0.091 & 0.141 & -0.189 & 0.371 & 1.07 & \\
\hline & ln biacromial breadth (mm) & $1.059^{*}$ & 0.378 & 0.308 & 1.810 & 1.689 & \\
\hline & Constant & 0.536 & 1.234 & -1.917 & 2.988 & & \\
\hline & Sex & $0.414^{\dagger}$ & 0.044 & 0.327 & 0.501 & 1.072 & \\
\hline 17 & Age & $-0.012^{\dagger}$ & 0.003 & -0.018 & -0.005 & 1.101 & 0.56 \\
\hline & Physical activity level & 0.053 & 0.139 & -0.224 & 0.329 & 1.056 & \\
\hline & In bitrochanteric breadth $(\mathrm{mm})$ & $0.971^{*}$ & 0.325 & 0.324 & 1.617 & 1.091 & \\
\hline & Constant & $2.339^{*}$ & 0.669 & 1.009 & 3.668 & & \\
\hline & Sex & $0.337^{\dagger}$ & 0.054 & 0.231 & 0.444 & 1.593 & \\
\hline 18 & Age & $-0.013^{\dagger}$ & 0.003 & -0.019 & -0.007 & 1.043 & 0.56 \\
\hline & Physical activity level & 0.050 & 0.139 & -0.228 & 0.327 & 1.056 & \\
\hline & ln bimalleolar breadth ( $\mathrm{mm})$ & $0.915^{*}$ & 0.313 & 0.292 & 1.537 & 1.538 & \\
\hline & Constant & $3.443^{\dagger}$ & 0.565 & 2.32 & 4.566 & & \\
\hline & Sex & $0.375^{\dagger}$ & 0.059 & 0.259 & 0.492 & 1.792 & \\
\hline 19 & Age & $-0.014^{\dagger}$ & 0.003 & -0.020 & -0.007 & 1.037 & 0.53 \\
\hline & Physical activity level & 0.029 & 0.145 & -0.260 & 0.317 & 1.065 & \\
\hline & ln elbow breadth (mm) & 0.375 & 0.269 & -0.159 & 0.908 & 1.704 & \\
\hline & Constant & $3.190^{\dagger}$ & 0.528 & 2.141 & 4.239 & & \\
\hline & Sex & $0.355^{\dagger}$ & 0.057 & 0.242 & 0.468 & 1.715 & \\
\hline 20 & Age & $-0.014^{\dagger}$ & 0.003 & -0.021 & -0.008 & 1.018 & 0.54 \\
\hline & Physical activity level & 0.036 & 0.143 & -0.248 & 0.319 & 1.058 & \\
\hline & ln wrist breadth (mm) & $0.576^{*}$ & 0.277 & 0.026 & 1.125 & 1.638 & \\
\hline & Constant & $2.929^{*}$ & 0.855 & 1.230 & 4.628 & & \\
\hline & Sex & $0.384^{\dagger}$ & 0.054 & 0.278 & 0.491 & 1.504 & \\
\hline 21 & Age & $-0.013^{\dagger}$ & 0.003 & -0.02 & -0.007 & 1.068 & 0.53 \\
\hline & Physical activity level & 0.049 & 0.144 & -0.238 & 0.336 & 1.056 & \\
\hline & ln chest breadth (mm) & 0.338 & 0.228 & -0.114 & 0.791 & 1.468 & \\
\hline & Constant & $2.709^{\dagger}$ & 0.656 & 1.407 & 4.012 & & \\
\hline & Sex & $0.384^{\dagger}$ & 0.048 & 0.289 & 0.479 & 1.243 & \\
\hline 22 & Age & $-0.011^{*}$ & 0.003 & -0.018 & -0.004 & 1.207 & 0.55 \\
\hline & Physical activity level & 0.056 & 0.142 & -0.226 & 0.337 & 1.057 & \\
\hline & ln body mass*height $(\mathrm{kg} * \mathrm{~m})$ & $0.256^{*}$ & 0.108 & 0.042 & 0.470 & 1.339 & \\
\hline & Constant & $3.641^{\dagger}$ & 0.345 & 2.955 & 4.327 & & \\
\hline & Sex & $0.388^{\dagger}$ & 0.048 & 0.293 & 0.483 & 1.222 & \\
\hline 23 & Age & $-0.011^{*}$ & 0.003 & -0.018 & -0.005 & 1.203 & 0.54 \\
\hline & Physical activity level & 0.057 & 0.142 & -0.225 & 0.339 & 1.057 & \\
\hline & $\ln \mathrm{SA}\left(\mathrm{m}^{2}\right)$ & $0.489^{*}$ & 0.214 & 0.064 & 0.915 & 1.317 & \\
\hline & Constant & $3.417^{\dagger}$ & 0.696 & 2.035 & 4.799 & & \\
\hline 24 & Sex & $0.405^{\dagger}$ & 0.050 & 0.306 & 0.504 & 1.276 & 0.52 \\
\hline & Age & $-0.013^{\dagger}$ & 0.003 & -0.020 & -0.007 & 1.129 & \\
\hline
\end{tabular}


medRxiv preprint doi: https://doi.org/10.1101/2021.07.28.21261230; this version posted July 31, 2021. The copyright holder for this preprint (which was not certified by peer review) is the author/funder, who has granted medRxiv a license to display the preprint in perpetuity. It is made available under a CC-BY-NC-ND 4.0 International license.

\begin{tabular}{|c|c|c|c|c|c|c|c|}
\hline \multirow{2}{*}{$\begin{array}{c}\text { Linear } \\
\text { regression }\end{array}$} & \multirow{2}{*}{ Independent variables } & \multicolumn{2}{|c|}{ Regression coefficients } & \multicolumn{2}{|c|}{$95 \%$ CI of $\beta$} & \multirow{2}{*}{ VIF } & \multirow{2}{*}{$\begin{array}{c}\text { Adjusted } \\
\mathbf{R}^{2}\end{array}$} \\
\hline & & $\beta$ & Standard error & $\mathbf{L L}$ & $\mathbf{U L}$ & & \\
\hline & Physical activity level & 0.034 & 0.145 & -0.255 & 0.323 & 1.062 & \\
\hline & $\ln$ MAMC $(\mathrm{cm})$ & 0.208 & 0.186 & -0.162 & 0.579 & 1.292 & \\
\hline \multirow{5}{*}{25} & Constant & $3.773^{\dagger}$ & 0.427 & 2.925 & 4.622 & & \\
\hline & Sex & $0.414^{\dagger}$ & 0.047 & 0.321 & 0.508 & 1.141 & \\
\hline & Age & $-0.013^{\dagger}$ & 0.003 & -0.020 & -0.007 & 1.134 & 0.52 \\
\hline & Physical activity level & 0.034 & 0.145 & -0.255 & 0.323 & 1.063 & \\
\hline & ln CAMA $\left(\mathrm{cm}^{2}\right)$ & 0.083 & 0.076 & -0.067 & 0.233 & 1.180 & \\
\hline \multirow{5}{*}{26} & Constant & $4.087^{\dagger}$ & 0.375 & 3.342 & 4.832 & & \\
\hline & Sex & $0.432^{\dagger}$ & 0.051 & 0.331 & 0.532 & 1.299 & \\
\hline & Age & $-0.014^{\dagger}$ & 0.003 & -0.021 & -0.008 & 1.105 & 0.52 \\
\hline & Physical activity level & 0.047 & 0.146 & -0.243 & 0.337 & 1.057 & \\
\hline & $\ln \mathrm{AFA}\left(\mathrm{cm}^{2}\right)$ & 0.010 & 0.058 & -0.106 & 0.126 & 1.353 & \\
\hline \multirow{5}{*}{27} & Constant & 1.747 & 0.897 & -0.036 & 3.530 & & \\
\hline & Sex & $0.242^{*}$ & 0.080 & 0.083 & 0.401 & 3.528 & \\
\hline & Age & $-0.008^{*}$ & 0.004 & -0.016 & -0.001 & 1.529 & 0.56 \\
\hline & Physical activity level & 0.086 & 0.141 & -0.194 & 0.366 & 1.067 & \\
\hline & $\ln \mathrm{FFM}_{\text {(LEAN et al., } 1996)}(\mathrm{kg})$ & $0.531^{*}$ & 0.191 & 0.152 & 0.910 & 3.814 & \\
\hline \multirow{5}{*}{28} & Constant & $3.934^{\dagger}$ & 0.391 & 3.157 & 4.711 & & \\
\hline & Sex & $0.447^{\dagger}$ & 0.053 & 0.342 & 0.551 & 1.414 & \\
\hline & Age & $-0.014^{\dagger}$ & 0.003 & -0.021 & -0.008 & 1.059 & 0.52 \\
\hline & Physical activity level & 0.050 & 0.146 & -0.239 & 0.339 & 1.057 & \\
\hline & In fat mass(LEAN et al., 1996) $(\mathrm{kg})$ & 0.048 & 0.067 & -0.085 & 0.180 & 1.415 & \\
\hline \multirow{5}{*}{29} & Constant & $1.774^{*}$ & 0.861 & 0.063 & 3.485 & & \\
\hline & Sex & $0.298^{\dagger}$ & 0.063 & 0.174 & 0.423 & 2.181 & \\
\hline & Age & $-0.011^{*}$ & 0.003 & -0.018 & -0.004 & 1.174 & 0.56 \\
\hline & Physical activity level & 0.036 & 0.140 & -0.241 & 0.314 & 1.057 & \\
\hline & ln left arm LST $(\mathrm{g})$ & $0.290^{*}$ & 0.101 & 0.090 & 0.490 & 2.182 & \\
\hline \multirow{5}{*}{30} & Constant & $1.864^{*}$ & 0.917 & 0.042 & 3.685 & & \\
\hline & Sex & $0.310^{\dagger}$ & 0.063 & 0.184 & 0.436 & 2.187 & \\
\hline & Age & $-0.011^{*}$ & 0.003 & -0.018 & -0.004 & 1.258 & 0.55 \\
\hline & Physical activity level & 0.065 & 0.141 & -0.215 & 0.345 & 1.059 & \\
\hline & ln right arm LST (g) & $0.264^{*}$ & 0.102 & 0.061 & 0.467 & 2.282 & \\
\hline & Constant & 1.314 & 1.213 & -1.096 & 3.723 & & \\
\hline & Sex & $0.326^{\dagger}$ & 0.062 & 0.203 & 0.448 & 2.049 & \\
\hline 31 & Age & $-0.011^{*}$ & 0.004 & -0.018 & -0.004 & 1.275 & 0.55 \\
\hline & Physical activity level & 0.053 & 0.142 & -0.228 & 0.334 & 1.056 & \\
\hline & ln left leg LST $(\mathrm{g})$ & $0.296^{*}$ & 0.124 & 0.049 & 0.543 & 2.149 & \\
\hline & Constant & 1.202 & 1.271 & -1.324 & 3.728 & & \\
\hline & Sex & $0.319^{\dagger}$ & 0.064 & 0.192 & 0.446 & 2.211 & \\
\hline 32 & Age & $-0.011^{*}$ & 0.004 & -0.018 & -0.004 & 1.288 & 0.54 \\
\hline & Physical activity level & 0.077 & 0.142 & -0.205 & 0.360 & 1.065 & \\
\hline & ln right leg LST (g) & $0.305^{*}$ & 0.129 & 0.048 & 0.562 & 2.351 & \\
\hline & Constant & 1.418 & 0.995 & -0.559 & 3.394 & & \\
\hline & Sex & $0.295^{\dagger}$ & 0.064 & 0.167 & 0.422 & 2.287 & \\
\hline 33 & Age & $-0.010^{*}$ & 0.003 & -0.017 & -0.004 & 1.237 & 0.56 \\
\hline & Physical activity level & 0.053 & 0.140 & -0.225 & 0.331 & 1.056 & \\
\hline & $\ln$ arms LST $(\mathrm{g})$ & $0.299^{*}$ & 0.106 & 0.089 & 0.509 & 2.346 & \\
\hline & Constant & 0.879 & 1.365 & -1.835 & 3.592 & & \\
\hline & Sex & $0.317^{\dagger}$ & 0.064 & 0.190 & 0.443 & 2.194 & \\
\hline 34 & Age & $-0.011^{*}$ & 0.004 & -0.018 & -0.003 & 1.298 & 0.55 \\
\hline & Physical activity level & 0.067 & 0.142 & -0.215 & 0.348 & 1.06 & \\
\hline & ln legs LST $(\mathrm{g})$ & $0.317^{*}$ & 0.130 & 0.058 & 0.576 & 2.324 & \\
\hline & Constant & $2.917^{\dagger}$ & 0.534 & 1.856 & 3.979 & & \\
\hline & Sex & $0.303^{\dagger}$ & 0.065 & 0.174 & 0.432 & 2.299 & \\
\hline 35 & Age & $-0.010^{*}$ & 0.004 & -0.017 & -0.003 & 1.302 & 0.55 \\
\hline & Physical activity level & 0.064 & 0.141 & -0.216 & 0.344 & 1.058 & \\
\hline & $\ln$ ASM (kg) & $0.335^{*}$ & 0.127 & 0.082 & 0.588 & 2.422 & \\
\hline & Constant & $3.808^{\dagger}$ & 0.463 & 2.888 & 4.729 & & \\
\hline & Sex & $0.400^{\dagger}$ & 0.056 & 0.288 & 0.511 & 1.601 & \\
\hline 36 & Age & $-0.013^{\dagger}$ & 0.003 & -0.02 & -0.007 & 1.179 & 0.52 \\
\hline & Physical activity level & 0.061 & 0.146 & -0.23 & 0.352 & 1.070 & \\
\hline & ln ASM $/$ height ${ }^{2}\left(\mathrm{~kg} / \mathrm{m}^{2}\right)$ & 0.130 & 0.149 & -0.167 & 0.426 & 1.685 & \\
\hline & Constant & $2.112^{*}$ & 0.764 & 0.593 & 3.631 & & \\
\hline 37 & Sex & $0.375^{\dagger}$ & 0.051 & 0.273 & 0.477 & 1.503 & 0.58 \\
\hline & Age & $-0.009^{*}$ & 0.003 & -0.016 & -0.002 & 1.235 & \\
\hline
\end{tabular}


medRxiv preprint doi: https://doi.org/10.1101/2021.07.28.21261230; this version posted July 31, 2021. The copyright holder for this preprint (which was not certified by peer review) is the author/funder, who has granted medRxiv a license to display the preprint in perpetuity. It is made available under a CC-BY-NC-ND 4.0 International license.

\begin{tabular}{|c|c|c|c|c|c|c|c|}
\hline \multirow{4}{*}{$\begin{array}{c}\text { Linear } \\
\text { regression }\end{array}$} & \multirow{2}{*}{ Independent variables } & \multicolumn{2}{|c|}{ Regression coefficients } & \multicolumn{2}{|c|}{$95 \%$ CI of $\beta$} & \multirow{2}{*}{ VIF } & \multirow{2}{*}{$\begin{array}{c}\text { Adjusted } \\
\mathbf{R}^{2}\end{array}$} \\
\hline & & $\beta$ & Standard error & $\mathbf{L L}$ & $\mathbf{U L}$ & & \\
\hline & Physical activity level & 0.114 & 0.137 & -0.159 & 0.386 & 1.074 & \\
\hline & ln FFM(BAUMGARTNER et al., 1991) $(\mathrm{kg})$ & $0.412^{*}$ & 0.157 & 0.099 & 0.724 & 1.665 & \\
\hline \multirow{5}{*}{38} & Constant & $2.569^{\dagger}$ & 0.680 & 1.218 & 3.920 & & \\
\hline & Sex & $0.325^{\dagger}$ & 0.060 & 0.205 & 0.445 & 1.979 & \\
\hline & Age & $-0.011^{*}$ & 0.003 & -0.018 & -0.004 & 1.231 & 0.55 \\
\hline & Physical activity level & 0.059 & 0.141 & -0.222 & 0.339 & 1.057 & \\
\hline & $\ln \mathrm{FFM}_{(\mathrm{DXA})}(\mathrm{kg})$ & $0.356^{*}$ & 0.142 & 0.073 & 0.638 & 2.054 & \\
\hline \multirow{5}{*}{39} & Constant & $3.988^{\dagger}$ & 0.408 & 3.177 & 4.799 & & \\
\hline & Sex & $0.438^{\dagger}$ & 0.050 & 0.338 & 0.537 & 1.268 & \\
\hline & Age & $-0.014^{\dagger}$ & 0.003 & -0.021 & -0.008 & 1.077 & 0.52 \\
\hline & Physical activity level & 0.052 & 0.146 & -0.238 & 0.343 & 1.063 & \\
\hline & $\ln$ fat mass $(\mathrm{DXA})(\mathrm{kg})$ & 0.034 & 0.070 & -0.105 & 0.172 & 1.28 & \\
\hline \multicolumn{8}{|c|}{ Dependent variable: $1 R M_{\text {knee extensors }}(\ln \mathrm{kg}$ ) } \\
\hline \multirow{5}{*}{1} & Constant & $3.703^{*}$ & 1.198 & 1.322 & 6.083 & & \\
\hline & Sex & $0.501^{\dagger}$ & 0.083 & 0.336 & 0.666 & 1.129 & \\
\hline & Age & $-0.027^{\dagger}$ & 0.006 & -0.039 & -0.015 & 1.181 & 0.43 \\
\hline & Physical activity level & -0.018 & 0.257 & -0.530 & 0.493 & 1.059 & \\
\hline & ln body mass $(\mathrm{kg})$ & 0.436 & 0.219 & 0.001 & 0.871 & 1.218 & \\
\hline \multirow{5}{*}{2} & Constant & $4.152^{\dagger}$ & 0.693 & 2.774 & 5.530 & & \\
\hline & Sex & $0.305^{*}$ & 0.104 & 0.098 & 0.512 & 1.919 & \\
\hline & Age & $-0.025^{\dagger}$ & 0.006 & -0.037 & -0.013 & 1.143 & 0.48 \\
\hline & Physical activity level & -0.137 & 0.249 & -0.631 & 0.357 & 1.068 & \\
\hline & ln height $(\mathrm{m})$ & $3.047^{*}$ & 0.897 & 1.266 & 4.829 & 1.891 & \\
\hline \multirow{5}{*}{3} & Constant & $4.546^{*}$ & 1.609 & 1.35 & 7.743 & & \\
\hline & Sex & $0.506^{\dagger}$ & 0.092 & 0.323 & 0.688 & 1.334 & \\
\hline & Age & $-0.03^{\dagger}$ & 0.006 & -0.042 & -0.018 & 1.128 & 0.41 \\
\hline & Physical activity level & -0.049 & 0.262 & -0.569 & 0.471 & 1.056 & \\
\hline & ln forearm circumference $(\mathrm{cm})$ & 0.384 & 0.441 & -0.492 & 1.260 & 1.349 & \\
\hline & Constant & 1.472 & 1.788 & -2.081 & 5.025 & & \\
\hline & Sex & $0.511^{\dagger}$ & 0.080 & 0.352 & 0.671 & 1.084 & \\
\hline 4 & Age & $-0.026^{\dagger}$ & 0.006 & -0.038 & -0.014 & 1.186 & 0.45 \\
\hline & Physical activity level & 0.03 & 0.255 & -0.477 & 0.538 & 1.071 & \\
\hline & ln calf circumference $(\mathrm{cm})$ & $1.104^{*}$ & 0.431 & 0.247 & 1.962 & 1.195 & \\
\hline & Constant & 3.186 & 2.190 & -1.166 & 7.538 & & \\
\hline & Sex & $0.513^{\dagger}$ & 0.085 & 0.345 & 0.681 & 1.143 & \\
\hline 5 & Age & $-0.029^{\dagger}$ & 0.006 & -0.041 & -0.017 & 1.110 & 0.42 \\
\hline & Physical activity level & -0.038 & 0.261 & -0.555 & 0.48 & 1.057 & \\
\hline & ln chest circumference $(\mathrm{cm})$ & 0.558 & 0.442 & -0.32 & 1.436 & 1.162 & \\
\hline & Constant & $4.581^{*}$ & 1.553 & 1.496 & 7.667 & & \\
\hline & Sex & $0.524^{\dagger}$ & 0.084 & 0.356 & 0.692 & 1.128 & \\
\hline 6 & Age & $-0.031^{\dagger}$ & 0.006 & -0.042 & -0.019 & 1.045 & 0.41 \\
\hline & Physical activity level & -0.046 & 0.262 & -0.566 & 0.474 & 1.056 & \\
\hline & ln waist circumference $(\mathrm{cm})$ & 0.277 & 0.315 & -0.347 & 0.902 & 1.088 & \\
\hline & Constant & -3.215 & 3.276 & -9.725 & 3.295 & & \\
\hline & Sex & $0.360^{*}$ & 0.102 & 0.157 & 0.563 & 1.779 & \\
\hline 7 & Age & $-0.028^{\dagger}$ & 0.006 & -0.04 & -0.017 & 1.061 & 0.46 \\
\hline & Physical activity level & -0.094 & 0.252 & -0.595 & 0.408 & 1.061 & \\
\hline & ln knee height $(\mathrm{cm})$ & $2.284^{*}$ & 0.814 & 0.667 & 3.901 & 1.708 & \\
\hline & Constant & -6.682 & 3.473 & -13.584 & 0.219 & & \\
\hline & Sex & $0.324^{*}$ & 0.097 & 0.130 & 0.517 & 1.705 & \\
\hline 8 & Age & $-0.024^{\dagger}$ & 0.006 & -0.036 & -0.012 & 1.161 & 0.49 \\
\hline & Physical activity level & 0.013 & 0.246 & -0.475 & 0.501 & 1.061 & \\
\hline & ln half arm span $(\mathrm{cm})$ & $2.728^{\dagger}$ & 0.747 & 1.243 & 4.213 & 1.752 & \\
\hline & Constant & $5.136^{\dagger}$ & 0.651 & 3.842 & 6.430 & & \\
\hline & Sex & $0.647^{\dagger}$ & 0.101 & 0.447 & 0.847 & 1.640 & \\
\hline 9 & Age & $-0.03^{\dagger}$ & 0.006 & -0.041 & -0.018 & 1.058 & 0.43 \\
\hline & Physical activity level & -0.022 & 0.259 & -0.536 & 0.492 & 1.059 & \\
\hline & ln triceps skinfold (mm) & 0.179 & 0.103 & -0.025 & 0.383 & 1.637 & \\
\hline & Constant & $5.585^{\dagger}$ & 0.616 & 4.360 & 6.809 & & \\
\hline & Sex & $0.596^{\dagger}$ & 0.105 & 0.387 & 0.805 & 1.752 & \\
\hline 10 & Age & $-0.031^{\dagger}$ & 0.006 & -0.043 & -0.019 & 1.041 & 0.41 \\
\hline & Physical activity level & -0.011 & 0.265 & -0.538 & 0.516 & 1.086 & \\
\hline & ln biceps skinfold (mm) & 0.077 & 0.095 & -0.111 & 0.265 & 1.706 & \\
\hline 11 & Constant & $5.74^{\dagger}$ & 0.650 & 4.448 & 7.033 & & 0.41 \\
\hline
\end{tabular}


medRxiv preprint doi: https://doi.org/10.1101/2021.07.28.21261230; this version posted July 31, 2021. The copyright holder for this preprint (which was not certified by peer review) is the author/funder, who has granted medRxiv a license to display the preprint in perpetuity. It is made available under a CC-BY-NC-ND 4.0 International license.

\begin{tabular}{|c|c|c|c|c|c|c|c|}
\hline \multirow{6}{*}{$\begin{array}{c}\text { Linear } \\
\text { regression }\end{array}$} & \multirow{2}{*}{ Independent variables } & \multicolumn{2}{|c|}{ Regression coefficients } & \multicolumn{2}{|c|}{$95 \%$ CI of $\beta$} & \multirow{2}{*}{ VIF } & \multirow{2}{*}{$\begin{array}{c}\text { Adjusted } \\
\mathbf{R}^{2}\end{array}$} \\
\hline & & $\beta$ & Standard error & $\mathbf{L L}$ & $\mathbf{U L}$ & & \\
\hline & Sex & $0.563^{\dagger}$ & 0.103 & 0.358 & 0.767 & 1.660 & \\
\hline & Age & $-0.031^{\dagger}$ & 0.006 & -0.043 & -0.02 & 1.026 & \\
\hline & Physical activity level & -0.031 & 0.267 & -0.561 & 0.499 & 1.091 & \\
\hline & ln thigh skinfold (mm) & 0.032 & 0.095 & -0.158 & 0.222 & 1.595 & \\
\hline \multirow{5}{*}{12} & Constant & $5.806^{\dagger}$ & 0.604 & 4.606 & 7.007 & & \\
\hline & Sex & $0.556^{\dagger}$ & 0.105 & 0.347 & 0.765 & 1.737 & \\
\hline & Age & $-0.031^{\dagger}$ & 0.006 & -0.043 & -0.02 & 1.041 & 0.41 \\
\hline & Physical activity level & -0.044 & 0.263 & -0.566 & 0.479 & 1.06 & \\
\hline & ln medial calf skinfold (mm) & 0.018 & 0.082 & -0.146 & 0.181 & 1.711 & \\
\hline \multirow{5}{*}{13} & Constant & -0.722 & 2.76 & -6.207 & 4.763 & & \\
\hline & Sex & $0.417^{\dagger}$ & 0.095 & 0.228 & 0.605 & 1.506 & \\
\hline & Age & $-0.024^{\dagger}$ & 0.006 & -0.037 & -0.012 & 1.295 & 0.45 \\
\hline & Physical activity level & 0.023 & 0.256 & -0.486 & 0.532 & 1.070 & \\
\hline & ln biacromial breadth (mm) & $1.668^{*}$ & 0.687 & 0.304 & 3.033 & 1.689 & \\
\hline \multirow{5}{*}{14} & Constant & 3.329 & 2.313 & -1.267 & 7.924 & & \\
\hline & Sex & $0.532^{\dagger}$ & 0.082 & 0.369 & 0.695 & 1.072 & \\
\hline & Age & $-0.03^{\dagger}$ & 0.006 & -0.042 & -0.018 & 1.101 & 0.42 \\
\hline & Physical activity level & -0.043 & 0.261 & -0.561 & 0.476 & 1.056 & \\
\hline & ln bitrochanteric breadth ( $\mathrm{mm})$ & 0.688 & 0.610 & -0.523 & 1.900 & 1.091 & \\
\hline \multirow{5}{*}{15} & Constant & $3.52^{*}$ & 1.23 & 1.076 & 5.965 & & \\
\hline & Sex & $0.423^{\dagger}$ & 0.098 & 0.228 & 0.619 & 1.593 & \\
\hline & Age & $-0.03^{\dagger}$ & 0.006 & -0.041 & -0.018 & 1.043 & 0.44 \\
\hline & Physical activity level & -0.043 & 0.257 & -0.553 & 0.467 & 1.056 & \\
\hline & ln bimalleolar breadth (mm) & $1.204^{*}$ & 0.576 & 0.059 & 2.349 & 1.538 & \\
\hline \multirow{5}{*}{16} & Constant & $5.702^{\dagger}$ & 1.028 & 3.660 & 7.744 & & \\
\hline & Sex & $0.529^{\dagger}$ & 0.107 & 0.316 & 0.741 & 1.792 & \\
\hline & Age & $-0.031^{\dagger}$ & 0.006 & -0.043 & -0.02 & 1.037 & 0.41 \\
\hline & Physical activity level & -0.052 & 0.264 & -0.576 & 0.472 & 1.065 & \\
\hline & ln elbow breadth $(\mathrm{mm})$ & 0.096 & 0.489 & -0.874 & 1.067 & 1.704 & \\
\hline \multirow{5}{*}{17} & Constant & $5.159^{\dagger}$ & 0.969 & 3.234 & 7.083 & & \\
\hline & Sex & $0.486^{\dagger}$ & 0.104 & 0.279 & 0.693 & 1.715 & \\
\hline & Age & $-0.031^{\dagger}$ & 0.006 & -0.043 & -0.02 & 1.018 & 0.41 \\
\hline & Physical activity level & -0.056 & 0.262 & -0.576 & 0.465 & 1.058 & \\
\hline & ln wrist breadth (mm) & 0.440 & 0.508 & -0.568 & 1.449 & 1.638 & \\
\hline & Constant & 3.383 & 2.075 & -0.74 & 7.506 & & \\
\hline & Sex & $0.469^{\dagger}$ & 0.101 & 0.269 & 0.669 & 1.616 & \\
\hline 18 & Age & $-0.029^{\dagger}$ & 0.006 & -0.041 & -0.016 & 1.178 & 0.42 \\
\hline & Physical activity level & -0.024 & 0.261 & -0.543 & 0.494 & 1.061 & \\
\hline & ln chest breadth (mm) & 0.684 & 0.552 & -0.413 & 1.781 & 1.679 & \\
\hline & Constant & $3.212^{*}$ & 1.176 & 0.875 & 5.55 & & \\
\hline & Sex & $0.460^{\dagger}$ & 0.086 & 0.289 & 0.631 & 1.243 & \\
\hline 19 & Age & $-0.025^{\dagger}$ & 0.006 & -0.038 & -0.013 & 1.207 & 0.45 \\
\hline & Physical activity level & -0.030 & 0.254 & -0.535 & 0.475 & 1.057 & \\
\hline & ln body mass*height $(\mathrm{kg} * \mathrm{~m})$ & $0.480^{*}$ & 0.193 & 0.097 & 0.863 & 1.339 & \\
\hline & Constant & $4.952^{\dagger}$ & 0.619 & 3.722 & 6.183 & & \\
\hline & Sex & $0.467^{\dagger}$ & 0.086 & 0.297 & 0.637 & 1.222 & \\
\hline 20 & Age & $-0.026^{\dagger}$ & 0.006 & -0.038 & -0.013 & 1.203 & 0.45 \\
\hline & Physical activity level & -0.027 & 0.255 & -0.533 & 0.479 & 1.057 & \\
\hline & $\ln \mathrm{SA}\left(\mathrm{m}^{2}\right)$ & $0.925^{*}$ & 0.384 & 0.163 & 1.688 & 1.317 & \\
\hline & Constant & $5.277^{\dagger}$ & 1.258 & 2.777 & 7.777 & & \\
\hline & Sex & $0.523^{\dagger}$ & 0.090 & 0.344 & 0.702 & 1.276 & \\
\hline 21 & Age & $-0.031^{\dagger}$ & 0.006 & -0.043 & -0.018 & 1.129 & 0.41 \\
\hline & Physical activity level & -0.058 & 0.263 & -0.581 & 0.465 & 1.062 & \\
\hline & $\ln \operatorname{MAMC}(\mathrm{cm})$ & 0.176 & 0.337 & -0.494 & 0.846 & 1.292 & \\
\hline & Constant & $5.528^{\dagger}$ & 0.772 & 3.993 & 7.062 & & \\
\hline & Sex & $0.529^{\dagger}$ & 0.085 & 0.359 & 0.698 & 1.141 & \\
\hline 22 & Age & $-0.030^{\dagger}$ & 0.006 & -0.043 & -0.018 & 1.134 & 0.41 \\
\hline & Physical activity level & -0.059 & 0.263 & -0.582 & 0.463 & 1.063 & \\
\hline & $\ln$ CAMA $\left(\mathrm{cm}^{2}\right)$ & 0.082 & 0.137 & -0.19 & 0.353 & 1.18 & \\
\hline & Constant & 1.932 & 1.625 & -1.297 & 5.161 & & \\
\hline & Sex & 0.234 & 0.145 & -0.054 & 0.522 & 3.528 & \\
\hline 23 & Age & $-0.021^{*}$ & 0.007 & -0.035 & -0.008 & 1.529 & 0.45 \\
\hline & Physical activity level & 0.018 & 0.255 & -0.489 & 0.525 & 1.067 & \\
\hline & ln FFM (LEAN et al., 1996) $(\mathrm{kg})$ & $0.879^{*}$ & 0.346 & 0.192 & 1.566 & 3.814 & \\
\hline 24 & Constant & $3.787^{*}$ & 1.603 & 0.603 & 6.972 & & 0.42 \\
\hline
\end{tabular}


medRxiv preprint doi: https://doi.org/10.1101/2021.07.28.21261230; this version posted July 31, 2021. The copyright holder for this preprint (which was not certified by peer review) is the author/funder, who has granted medRxiv a license to display the preprint in perpetuity. It is made available under a CC-BY-NC-ND 4.0 International license .

\begin{tabular}{|c|c|c|c|c|c|c|c|}
\hline \multirow{2}{*}{$\begin{array}{c}\text { Linear } \\
\text { regression }\end{array}$} & \multirow{2}{*}{ Independent variables } & \multicolumn{2}{|c|}{ Regression coefficients } & \multicolumn{2}{|c|}{$95 \%$ CI of $\beta$} & \multirow{2}{*}{ VIF } & \multirow{2}{*}{$\begin{array}{c}\text { Adjusted } \\
\mathbf{R}^{2}\end{array}$} \\
\hline & & $\beta$ & Standard error & $\mathbf{L L}$ & $\mathbf{U L}$ & & \\
\hline & Sex & $0.427^{\dagger}$ & 0.117 & 0.195 & 0.659 & 2.181 & \\
\hline & Age & $-0.028^{\dagger}$ & 0.006 & -0.041 & -0.016 & 1.174 & \\
\hline & Physical activity level & -0.056 & 0.260 & -0.573 & 0.460 & 1.057 & \\
\hline & ln left arm LST (g) & 0.257 & 0.188 & -0.115 & 0.630 & 2.182 & \\
\hline \multirow{5}{*}{25} & Constant & $3.986^{*}$ & 1.698 & 0.613 & 7.359 & & \\
\hline & Sex & $0.444^{\dagger}$ & 0.117 & 0.211 & 0.677 & 2.187 & \\
\hline & Age & $-0.028^{\dagger}$ & 0.006 & -0.041 & -0.015 & 1.258 & 0.42 \\
\hline & Physical activity level & -0.032 & 0.261 & -0.550 & 0.487 & 1.059 & \\
\hline & ln right arm LST (g) & 0.22 & 0.189 & -0.155 & 0.596 & 2.282 & \\
\hline \multirow{5}{*}{26} & Constant & -0.207 & 2.151 & -4.481 & 4.066 & & \\
\hline & Sex & $0.322^{*}$ & 0.109 & 0.105 & 0.539 & 2.049 & \\
\hline & Age & $-0.023^{\dagger}$ & 0.006 & -0.036 & -0.011 & 1.275 & 0.46 \\
\hline & Physical activity level & -0.033 & 0.251 & -0.532 & 0.466 & 1.056 & \\
\hline & ln left leg LST $(\mathrm{g})$ & $0.639^{*}$ & 0.220 & 0.202 & 1.077 & 2.149 & \\
\hline \multirow{5}{*}{27} & Constant & 0.338 & 2.280 & -4.192 & 4.868 & & \\
\hline & Sex & $0.336^{*}$ & 0.115 & 0.108 & 0.564 & 2.211 & \\
\hline & Age & $-0.024^{\dagger}$ & 0.006 & -0.037 & -0.012 & 1.288 & 0.45 \\
\hline & Physical activity level & 0.011 & 0.255 & -0.496 & 0.518 & 1.065 & \\
\hline & ln right leg LST (g) & $0.577^{*}$ & 0.232 & 0.116 & 1.037 & 2.351 & \\
\hline \multirow{5}{*}{28} & Constant & 3.537 & 1.851 & -0.142 & 7.215 & & \\
\hline & Sex & $0.427^{*}$ & 0.120 & 0.189 & 0.665 & 2.287 & \\
\hline & Age & $-0.028^{\dagger}$ & 0.006 & -0.041 & -0.015 & 1.237 & 0.42 \\
\hline & Physical activity level & -0.042 & 0.260 & -0.559 & 0.475 & 1.056 & \\
\hline & $\ln$ arms LST $(\mathrm{g})$ & 0.258 & 0.197 & -0.132 & 0.649 & 2.346 & \\
\hline \multirow{5}{*}{29} & Constant & -0.695 & 2.435 & -5.534 & 4.144 & & \\
\hline & Sex & $0.317^{*}$ & 0.114 & 0.092 & 0.543 & 2.194 & \\
\hline & Age & $-0.023^{\dagger}$ & 0.006 & -0.036 & -0.011 & 1.298 & 0.46 \\
\hline & Physical activity level & -0.006 & 0.253 & -0.508 & 0.495 & 1.060 & \\
\hline & ln legs LST $(\mathrm{g})$ & $0.641^{*}$ & 0.233 & 0.178 & 1.103 & 2.324 & \\
\hline \multirow{5}{*}{30} & Constant & $3.848^{\dagger}$ & 0.966 & 1.929 & 5.768 & & \\
\hline & Sex & $0.333^{*}$ & 0.117 & 0.100 & 0.566 & 2.299 & \\
\hline & Age & $-0.024^{\dagger}$ & 0.006 & -0.037 & -0.012 & 1.302 & 0.45 \\
\hline & Physical activity level & -0.018 & 0.255 & -0.524 & 0.488 & 1.058 & \\
\hline & $\ln \mathrm{ASM}(\mathrm{kg})$ & $0.560^{*}$ & 0.231 & 0.102 & 1.018 & 2.422 & \\
\hline & Constant & $5.305^{\dagger}$ & 0.834 & 3.648 & 6.961 & & \\
\hline & Sex & $0.492^{\dagger}$ & 0.101 & 0.292 & 0.692 & 1.601 & \\
\hline 31 & Age & $-0.030^{\dagger}$ & 0.006 & -0.042 & -0.017 & 1.179 & 0.41 \\
\hline & Physical activity level & -0.021 & 0.263 & -0.545 & 0.502 & 1.070 & \\
\hline & ln ASM $/$ height $^{2}\left(\mathrm{~kg} / \mathrm{m}^{2}\right)$ & 0.231 & 0.268 & -0.302 & 0.764 & 1.685 & \\
\hline & Constant & $2.917^{*}$ & 1.443 & 0.05 & 5.785 & & \\
\hline & Sex & $0.415^{\dagger}$ & 0.097 & 0.222 & 0.607 & 1.503 & \\
\hline 32 & Age & $-0.027^{\dagger}$ & 0.006 & -0.039 & -0.014 & 1.235 & 0.45 \\
\hline & Physical activity level & 0.012 & 0.259 & -0.502 & 0.526 & 1.074 & \\
\hline & In FFM(BAUMGARTNER et al., 1991) $(\mathrm{kg})$ & $0.671^{*}$ & 0.297 & 0.081 & 1.262 & 1.665 & \\
\hline & Constant & $4.193^{*}$ & 1.251 & 1.709 & 6.678 & & \\
\hline & Sex & $0.431^{\dagger}$ & 0.111 & 0.21 & 0.652 & 1.979 & \\
\hline 33 & Age & $-0.028^{\dagger}$ & 0.006 & -0.040 & -0.015 & 1.231 & 0.42 \\
\hline & Physical activity level & -0.034 & 0.260 & -0.550 & 0.482 & 1.057 & \\
\hline & $\ln \mathrm{FFM}_{(\mathrm{DXA})}(\mathrm{kg})$ & 0.384 & 0.261 & -0.136 & 0.903 & 2.054 & \\
\hline & Dependent va & iable: knee & ension $P T^{60 \%}(\ln$ & & & & \\
\hline & Constant & $4.174^{*}$ & 1.230 & 1.731 & 6.618 & & \\
\hline & Sex & $0.520^{\dagger}$ & 0.085 & 0.351 & 0.689 & 1.129 & \\
\hline 1 & Age & $-0.025^{\dagger}$ & 0.006 & -0.038 & -0.013 & 1.181 & 0.40 \\
\hline & Physical activity level & 0.259 & 0.264 & -0.267 & 0.784 & 1.059 & \\
\hline & ln body mass (kg) & 0.367 & 0.225 & -0.08 & 0.814 & 1.218 & \\
\hline & Constant & $4.153^{\dagger}$ & 0.702 & 2.759 & 5.548 & & \\
\hline & Sex & $0.301^{*}$ & 0.105 & 0.091 & 0.510 & 1.919 & \\
\hline 2 & Age & $-0.022^{\dagger}$ & 0.006 & -0.034 & -0.010 & 1.143 & 0.46 \\
\hline & Physical activity level & 0.138 & 0.252 & -0.362 & 0.638 & 1.068 & \\
\hline & ln height $(\mathrm{m})$ & $3.268^{*}$ & 0.908 & 1.465 & 5.071 & 1.891 & \\
\hline & Constant & $3.966^{*}$ & 1.631 & 0.725 & 7.206 & & \\
\hline 3 & Sex & $0.499^{\dagger}$ & 0.093 & 0.314 & 0.684 & 1.334 & $0 \rightarrow 0$ \\
\hline 3 & Age & $-0.026^{\dagger}$ & 0.006 & -0.039 & -0.014 & 1.128 & 0.40 \\
\hline & Physical activity level & 0.232 & 0.265 & -0.295 & 0.759 & 1.056 & \\
\hline
\end{tabular}


medRxiv preprint doi: https://doi.org/10.1101/2021.07.28.21261230; this version posted July 31, 2021. The copyright holder for this preprint (which was not certified by peer review) is the author/funder, who has granted medRxiv a license to display the preprint in perpetuity. It is made available under a CC-BY-NC-ND 4.0 International license.

\begin{tabular}{|c|c|c|c|c|c|c|c|}
\hline \multirow{3}{*}{$\begin{array}{c}\text { Linear } \\
\text { regression }\end{array}$} & \multirow{2}{*}{ Independent variables } & \multicolumn{2}{|c|}{ Regression coefficients } & \multicolumn{2}{|c|}{$95 \%$ CI of $\beta$} & \multirow{2}{*}{ VIF } & \multirow{2}{*}{$\begin{array}{c}\text { Adjusted } \\
\mathbf{R}^{2}\end{array}$} \\
\hline & & $\beta$ & Standard error & $\mathbf{L L}$ & $\mathbf{U L}$ & & \\
\hline & ln forearm circumference $(\mathrm{cm})$ & 0.588 & 0.447 & -0.300 & 1.476 & 1.349 & \\
\hline \multirow{5}{*}{4} & Constant & 1.299 & 1.816 & -2.308 & 4.907 & & \\
\hline & Sex & $0.522^{\dagger}$ & 0.082 & 0.360 & 0.683 & 1.084 & \\
\hline & Age & $-0.023^{\dagger}$ & 0.006 & -0.035 & -0.010 & 1.186 & 0.43 \\
\hline & Physical activity level & 0.317 & 0.259 & -0.198 & 0.832 & 1.071 & \\
\hline & ln calf circumference $(\mathrm{cm})$ & $1.179^{*}$ & 0.438 & 0.309 & 2.05 & 1.195 & \\
\hline \multirow{5}{*}{5} & Constant & 2.509 & 2.220 & -1.901 & 6.920 & & \\
\hline & Sex & $0.517^{\dagger}$ & 0.086 & 0.347 & 0.687 & 1.143 & \\
\hline & Age & $-0.026^{\dagger}$ & 0.006 & -0.038 & -0.014 & 1.110 & 0.40 \\
\hline & Physical activity level & 0.247 & 0.264 & -0.278 & 0.771 & 1.057 & \\
\hline & ln chest circumference $(\mathrm{cm})$ & 0.724 & 0.448 & -0.166 & 1.614 & 1.162 & \\
\hline \multirow{5}{*}{6} & Constant & $4.120^{*}$ & 1.576 & 0.990 & 7.251 & & \\
\hline & Sex & $0.528^{\dagger}$ & 0.086 & 0.358 & 0.698 & 1.128 & \\
\hline & Age & $-0.028^{\dagger}$ & 0.006 & -0.04 & -0.016 & 1.045 & 0.40 \\
\hline & Physical activity level & 0.236 & 0.265 & -0.291 & 0.763 & 1.056 & \\
\hline & ln waist circumference $(\mathrm{cm})$ & 0.403 & 0.319 & -0.231 & 1.037 & 1.088 & \\
\hline \multirow{5}{*}{7} & Constant & -1.243 & 3.396 & -7.991 & 5.504 & & \\
\hline & Sex & $0.409^{\dagger}$ & 0.106 & 0.199 & 0.620 & 1.779 & \\
\hline & Age & $-0.026^{\dagger}$ & 0.006 & -0.038 & -0.015 & 1.061 & 0.42 \\
\hline & Physical activity level & 0.197 & 0.262 & -0.322 & 0.717 & 1.061 & \\
\hline & ln knee height $(\mathrm{cm})$ & $1.820^{*}$ & 0.843 & 0.144 & 3.496 & 1.708 & \\
\hline \multirow{5}{*}{8} & Constant & -1.433 & 3.711 & -8.807 & 5.940 & & \\
\hline & Sex & $0.425^{\dagger}$ & 0.104 & 0.219 & 0.632 & 1.705 & \\
\hline & Age & $-0.025^{\dagger}$ & 0.006 & -0.037 & -0.012 & 1.161 & 0.41 \\
\hline & Physical activity level & 0.270 & 0.262 & -0.252 & 0.791 & 1.061 & \\
\hline & ln half arm span $(\mathrm{cm})$ & $1.615^{*}$ & 0.799 & 0.029 & 3.202 & 1.752 & \\
\hline \multirow{5}{*}{9} & Constant & $5.377^{\dagger}$ & 0.667 & 4.051 & 6.702 & & \\
\hline & Sex & $0.643^{\dagger}$ & 0.103 & 0.438 & 0.848 & 1.640 & \\
\hline & Age & $-0.027^{\dagger}$ & 0.006 & -0.039 & -0.015 & 1.058 & 0.40 \\
\hline & Physical activity level & 0.256 & 0.265 & -0.271 & 0.782 & 1.059 & \\
\hline & ln triceps skinfold (mm) & 0.152 & 0.105 & -0.057 & 0.361 & 1.637 & \\
\hline & Constant & $5.780^{\dagger}$ & 0.629 & 4.530 & 7.030 & & \\
\hline & Sex & $0.596^{\dagger}$ & 0.108 & 0.382 & 0.809 & 1.752 & \\
\hline 10 & Age & $-0.028^{\dagger}$ & 0.006 & -0.04 & -0.016 & 1.041 & 0.39 \\
\hline & Physical activity level & 0.262 & 0.271 & -0.277 & 0.800 & 1.086 & \\
\hline & ln biceps skinfold (mm) & 0.059 & 0.097 & -0.133 & 0.251 & 1.706 & \\
\hline & Constant & $6.422^{\dagger}$ & 0.660 & 5.111 & 7.733 & & \\
\hline & Sex & $0.492^{\dagger}$ & 0.104 & 0.285 & 0.700 & 1.660 & \\
\hline 11 & Age & $-0.030^{\dagger}$ & 0.006 & -0.041 & -0.018 & 1.026 & 0.39 \\
\hline & Physical activity level & 0.186 & 0.271 & -0.351 & 0.724 & 1.091 & \\
\hline & ln thigh skinfold (mm) & -0.096 & 0.097 & -0.288 & 0.096 & 1.595 & \\
\hline & Constant & $5.993^{\dagger}$ & 0.616 & 4.77 & 7.217 & & \\
\hline & Sex & $0.557^{\dagger}$ & 0.107 & 0.344 & 0.770 & 1.737 & \\
\hline 12 & Age & $-0.029^{\dagger}$ & 0.006 & -0.041 & -0.017 & 1.041 & 0.39 \\
\hline & Physical activity level & 0.235 & 0.268 & -0.298 & 0.768 & 1.060 & \\
\hline & ln medial calf skinfold (mm) & 0.003 & 0.084 & -0.164 & 0.169 & 1.711 & \\
\hline & Constant & -2.485 & 2.757 & -7.964 & 2.994 & & \\
\hline & Sex & $0.393^{\dagger}$ & 0.095 & 0.205 & 0.582 & 1.506 & \\
\hline 13 & Age & $-0.020^{*}$ & 0.006 & -0.032 & -0.007 & 1.295 & 0.45 \\
\hline & Physical activity level & 0.325 & 0.256 & -0.183 & 0.833 & 1.070 & \\
\hline & ln biacromial breadth (mm) & $2.146^{*}$ & 0.686 & 0.783 & 3.509 & 1.689 & \\
\hline & Constant & 4.151 & 2.365 & -0.548 & 8.851 & & \\
\hline & Sex & $0.547^{\dagger}$ & 0.084 & 0.38 & 0.714 & 1.072 & \\
\hline 14 & Age & $-0.028^{\dagger}$ & 0.006 & -0.040 & -0.015 & 1.101 & 0.39 \\
\hline & Physical activity level & 0.238 & 0.267 & -0.293 & 0.768 & 1.056 & \\
\hline & ln bitrochanteric breadth $(\mathrm{mm})$ & 0.500 & 0.624 & -0.739 & 1.739 & 1.091 & \\
\hline & Constant & $2.982^{*}$ & 1.236 & 0.527 & 5.438 & & \\
\hline & Sex & $0.402^{\dagger}$ & 0.099 & 0.206 & 0.599 & 1.593 & \\
\hline 15 & Age & $-0.026^{\dagger}$ & 0.006 & -0.038 & -0.015 & 1.043 & 0.43 \\
\hline & Physical activity level & 0.239 & 0.258 & -0.273 & 0.751 & 1.056 & \\
\hline & ln bimalleolar breadth $(\mathrm{mm})$ & $1.543^{*}$ & 0.579 & 0.393 & 2.693 & 1.538 & \\
\hline & Constant & $5.825^{\dagger}$ & 1.047 & 3.744 & 7.906 & & \\
\hline & Sex & $0.541^{\dagger}$ & 0.109 & 0.324 & 0.757 & 1.792 & \\
\hline 16 & Age & $-0.029^{\dagger}$ & 0.006 & -0.041 & -0.017 & 1.037 & 0.39 \\
\hline & Physical activity level & 0.230 & 0.269 & -0.304 & 0.764 & 1.065 & \\
\hline
\end{tabular}


medRxiv preprint doi: https://doi.org/10.1101/2021.07.28.21261230; this version posted July 31, 2021. The copyright holder for this preprint (which was not certified by peer review) is the author/funder, who has granted medRxiv a license to display the preprint in perpetuity. It is made available under a CC-BY-NC-ND 4.0 International license.

\begin{tabular}{|c|c|c|c|c|c|c|c|}
\hline \multirow{2}{*}{$\begin{array}{c}\text { Linear } \\
\text { regression }\end{array}$} & \multirow{2}{*}{ Independent variables } & \multicolumn{2}{|c|}{ Regression coefficients } & \multicolumn{2}{|c|}{$95 \%$ CI of $\beta$} & \multirow{2}{*}{ VIF } & \multirow{2}{*}{$\begin{array}{c}\text { Adjusted } \\
\mathbf{R}^{2}\end{array}$} \\
\hline & & $\beta$ & Standard error & $\mathbf{L L}$ & $\mathbf{U L}$ & & \\
\hline & ln elbow breadth (mm) & 0.098 & 0.498 & -0.891 & 1.087 & 1.704 & \multirow{6}{*}{0.40} \\
\hline \multirow{5}{*}{17} & Constant & $4.697^{\dagger}$ & 0.978 & 2.754 & 6.640 & & \\
\hline & Sex & $0.453^{\dagger}$ & 0.105 & 0.244 & 0.662 & 1.715 & \\
\hline & Age & $-0.029^{\dagger}$ & 0.006 & -0.04 & -0.017 & 1.018 & \\
\hline & Physical activity level & 0.219 & 0.264 & -0.306 & 0.744 & 1.058 & \\
\hline & ln wrist breadth (mm) & 0.800 & 0.513 & -0.218 & 1.819 & 1.638 & \\
\hline \multirow{5}{*}{18} & Constant & 0.890 & 2.058 & -3.200 & 4.980 & & \multirow{5}{*}{0.43} \\
\hline & Sex & $0.405^{\dagger}$ & 0.100 & 0.206 & 0.603 & 1.616 & \\
\hline & Age & $-0.023^{\dagger}$ & 0.006 & -0.035 & -0.011 & 1.178 & \\
\hline & Physical activity level & 0.281 & 0.259 & -0.234 & 0.795 & 1.061 & \\
\hline & ln chest breadth (mm) & $1.402^{*}$ & 0.548 & 0.314 & 2.490 & 1.679 & \\
\hline \multirow{5}{*}{19} & Constant & $3.591^{*}$ & 1.208 & 1.191 & 5.990 & & \multirow{5}{*}{0.42} \\
\hline & Sex & $0.480^{\dagger}$ & 0.088 & 0.304 & 0.656 & 1.243 & \\
\hline & Age & $-0.023^{\dagger}$ & 0.006 & -0.036 & -0.011 & 1.207 & \\
\hline & Physical activity level & 0.250 & 0.261 & -0.268 & 0.769 & 1.057 & \\
\hline & ln body mass*height $(\mathrm{kg} * \mathrm{~m})$ & $0.435^{*}$ & 0.198 & 0.041 & 0.828 & 1.339 & \\
\hline & Constant & $5.174^{\dagger}$ & 0.636 & 3.911 & 6.438 & & \\
\hline & Sex & $0.487^{\dagger}$ & 0.088 & 0.313 & 0.662 & 1.222 & \\
\hline 20 & Age & $-0.024^{\dagger}$ & 0.006 & -0.036 & -0.011 & 1.203 & 0.41 \\
\hline & Physical activity level & 0.252 & 0.261 & -0.267 & 0.772 & 1.057 & \\
\hline & $\ln \mathrm{SA}\left(\mathrm{m}^{2}\right)$ & $0.830^{*}$ & 0.394 & 0.047 & 1.613 & 1.317 & \\
\hline & Constant & $5.163^{\dagger}$ & 1.281 & 2.618 & 7.708 & & \\
\hline & Sex & $0.528^{\dagger}$ & 0.092 & 0.345 & 0.710 & 1.276 & \\
\hline 21 & Age & $-0.028^{\dagger}$ & 0.006 & -0.040 & -0.015 & 1.129 & 0.39 \\
\hline & Physical activity level & 0.219 & 0.268 & -0.313 & 0.752 & 1.062 & \\
\hline & $\ln \operatorname{MAMC}(\mathrm{cm})$ & 0.246 & 0.343 & -0.436 & 0.928 & 1.292 & \\
\hline & Constant & $5.547^{\dagger}$ & 0.786 & 3.985 & 7.108 & & \\
\hline & Sex & $0.537^{\dagger}$ & 0.087 & 0.365 & 0.709 & 1.141 & \\
\hline 22 & Age & $-0.027^{\dagger}$ & 0.006 & -0.04 & -0.015 & 1.134 & 0.39 \\
\hline & Physical activity level & 0.218 & 0.268 & -0.313 & 0.750 & 1.063 & \\
\hline & ln CAMA $\left(\mathrm{cm}^{2}\right)$ & 0.107 & 0.139 & -0.17 & 0.383 & 1.180 & \\
\hline & Constant & $3.606^{*}$ & 1.695 & 0.239 & 6.973 & & \\
\hline & Sex & $0.367^{*}$ & 0.151 & 0.067 & 0.667 & 3.528 & \\
\hline 23 & Age & $-0.023^{*}$ & 0.007 & -0.037 & -0.008 & 1.529 & 0.40 \\
\hline & Physical activity level & 0.274 & 0.266 & -0.254 & 0.802 & 1.067 & \\
\hline & ln FFM (LEAN et al., 1996) $(\mathrm{kg})$ & 0.534 & 0.36 & -0.182 & 1.250 & 3.814 & \\
\hline & Constant & $3.378^{*}$ & 1.624 & 0.151 & 6.606 & & \\
\hline & Sex & $0.410^{*}$ & 0.118 & 0.175 & 0.645 & 2.181 & \\
\hline 24 & Age & $-0.025^{\dagger}$ & 0.006 & -0.038 & -0.013 & 1.174 & 0.41 \\
\hline & Physical activity level & 0.223 & 0.264 & -0.301 & 0.746 & 1.057 & \\
\hline & ln left arm LST (g) & 0.323 & 0.19 & -0.054 & 0.701 & 2.182 & \\
\hline & Constant & $5.207^{*}$ & 1.741 & 1.747 & 8.666 & & \\
\hline & Sex & $0.513^{\dagger}$ & 0.120 & 0.274 & 0.752 & 2.187 & \\
\hline 25 & Age & $-0.028^{\dagger}$ & 0.007 & -0.041 & -0.014 & 1.258 & 0.39 \\
\hline & Physical activity level & 0.241 & 0.268 & -0.291 & 0.773 & 1.059 & \\
\hline & ln right arm LST $(\mathrm{g})$ & 0.093 & 0.194 & -0.292 & 0.478 & 2.282 & \\
\hline & Constant & 1.938 & 2.25 & -2.533 & 6.409 & & \\
\hline & Sex & $0.407^{*}$ & 0.114 & 0.18 & 0.634 & 2.049 & \\
\hline 26 & Age & $-0.024^{*}$ & 0.007 & -0.037 & -0.010 & 1.275 & 0.41 \\
\hline & Physical activity level & 0.244 & 0.263 & -0.278 & 0.766 & 1.056 & \\
\hline & ln left leg LST (g) & 0.427 & 0.23 & -0.031 & 0.885 & 2.149 & \\
\hline & Constant & 1.383 & 2.35 & -3.286 & 6.052 & & \\
\hline & Sex & $0.383^{*}$ & 0.118 & 0.147 & 0.618 & 2.211 & \\
\hline 27 & Age & $-0.023^{*}$ & 0.007 & -0.036 & -0.010 & 1.288 & 0.41 \\
\hline & Physical activity level & 0.283 & 0.263 & -0.240 & 0.805 & 1.065 & \\
\hline & ln right leg LST $(\mathrm{g})$ & $0.481^{*}$ & 0.239 & 0.006 & 0.956 & 2.351 & \\
\hline & Constant & $4.074^{*}$ & 1.893 & 0.313 & 7.835 & & \\
\hline & Sex & $0.460^{\dagger}$ & 0.122 & 0.217 & 0.703 & 2.287 & \\
\hline 28 & Age & $-0.026^{\dagger}$ & 0.007 & -0.039 & -0.013 & 1.237 & 0.39 \\
\hline & Physical activity level & 0.239 & 0.266 & -0.29 & 0.768 & 1.056 & \\
\hline & ln arms LST (g) & 0.213 & 0.201 & -0.187 & 0.612 & 2.346 & \\
\hline & Constant & 1.137 & 2.531 & -3.892 & 6.167 & & \\
\hline 20 & Sex & $0.388^{*}$ & 0.118 & 0.154 & 0.623 & 2.194 & ( 4 \\
\hline 29 & Age & $-0.023^{*}$ & 0.007 & -0.036 & -0.010 & 1.298 & 0.41 \\
\hline & Physical activity level & 0.264 & 0.263 & -0.257 & 0.786 & 1.060 & \\
\hline
\end{tabular}


medRxiv preprint doi: https://doi.org/10.1101/2021.07.28.21261230; this version posted July 31,2021 . The copyright holder for this preprint (which was not certified by peer review) is the author/funder, who has granted medRxiv a license to display the preprint in perpetuity.

It is made available under a CC-BY-NC-ND 4.0 International license .

\begin{tabular}{|c|c|c|c|c|c|c|c|}
\hline \multirow{3}{*}{$\begin{array}{c}\text { Linear } \\
\text { regression }\end{array}$} & \multirow{2}{*}{ Independent variables } & \multicolumn{2}{|c|}{ Regression coefficients } & \multicolumn{2}{|c|}{$95 \%$ CI of $\beta$} & \multirow{2}{*}{ VIF } & \multirow{2}{*}{$\begin{array}{c}\text { Adjusted } \\
\mathbf{R}^{2}\end{array}$} \\
\hline & & $\beta$ & Standard error & $\mathbf{L L}$ & $\mathbf{U L}$ & & \\
\hline & ln legs LST (g) & 0.474 & 0.242 & -0.006 & 0.955 & 2.324 & \\
\hline \multirow{5}{*}{30} & Constant & $4.476^{\dagger}$ & 0.999 & 2.491 & 6.462 & & \\
\hline & Sex & $0.397^{*}$ & 0.121 & 0.156 & 0.638 & 2.299 & \\
\hline & Age & $-0.023^{*}$ & 0.007 & -0.037 & -0.010 & 1.302 & 0.41 \\
\hline & Physical activity level & 0.257 & 0.263 & -0.267 & 0.780 & 1.058 & \\
\hline & ln ASM (kg) & 0.422 & 0.238 & -0.052 & 0.896 & 2.422 & \\
\hline \multirow{5}{*}{31} & Constant & $5.959^{\dagger}$ & 0.853 & 4.263 & 7.654 & & \\
\hline & Sex & $0.551^{\dagger}$ & 0.103 & 0.346 & 0.755 & 1.601 & \\
\hline & Age & $-0.029^{\dagger}$ & 0.006 & -0.042 & -0.016 & 1.179 & 0.39 \\
\hline & Physical activity level & 0.236 & 0.269 & -0.299 & 0.772 & 1.070 & \\
\hline & $\ln$ ASM $/$ height $^{2}\left(\mathrm{~kg} / \mathrm{m}^{2}\right)$ & 0.019 & 0.274 & -0.527 & 0.564 & 1.685 & \\
\hline \multirow{5}{*}{32} & Constant & $3.261^{*}$ & 1.486 & 0.306 & 6.215 & & \\
\hline & Sex & $0.443^{\dagger}$ & 0.100 & 0.245 & 0.642 & 1.503 & \\
\hline & Age & $-0.024^{*}$ & 0.007 & -0.037 & -0.011 & 1.235 & 0.41 \\
\hline & Physical activity level & 0.294 & 0.266 & -0.236 & 0.823 & 1.074 & \\
\hline & ln FFM (BAUMGARTNER et al., 1991) $(\mathrm{kg})$ & $0.612^{*}$ & 0.306 & 0.004 & 1.220 & 1.665 & \\
\hline \multirow{5}{*}{33} & Constant & $4.617^{*}$ & 1.280 & 2.074 & 7.160 & & \\
\hline & Sex & $0.463^{\dagger}$ & 0.114 & 0.237 & 0.689 & 1.979 & \\
\hline & Age & $-0.026^{\dagger}$ & 0.007 & -0.039 & -0.013 & 1.231 & 0.40 \\
\hline & Physical activity level & 0.245 & 0.266 & -0.283 & 0.773 & 1.057 & \\
\hline & $\ln \mathrm{FFM}_{(\mathrm{DXA})}(\mathrm{kg})$ & 0.316 & 0.268 & -0.216 & 0.848 & 2.054 & \\
\hline
\end{tabular}

$\mathrm{p}<0,05$ and ${ }^{\dagger} \mathrm{p}<0,001$ (statistically significant $\beta$ )

Note: $\operatorname{sex}=0$ for women and 1 for men; age in years; physical activity level=0 for inactive and 1 for active; $\ln =$ natural $\operatorname{logarithm} ; \beta=$ coefficient of regression; $\mathrm{CI}=$ confidence interval; $\mathrm{LL}=$ lower limit; $\mathrm{UL}=$ upper limit; $\mathrm{VIF}=$ variance inflation factor; $\mathrm{R}^{2}=$ coefficient of determination; $\mathrm{BMI}=$ body mass index; $\mathrm{SA}=$ surface area of human body; $\mathrm{MAMC}=$ mid-arm muscle circumference; $\mathrm{CAMA}=$ corrected arm muscle area; $\mathrm{AFA}=$ arm fat area; FFM=Fat-free mass; LST=lean soft tissue; ASM=appendicular skeletal muscle mass; DXA=Dual-energy X-ray absorptiometry. 
SUPPLEMENT C - Cut-off points to identify muscle weakness in older adults of the handgrip strength (HGS), one maximum repetition measurement for knee extensors $\left(1 \mathrm{RM}_{\text {knee extensors }}\right)$ and isokinetic knee extension peak torque at $60^{\circ} / \mathrm{s}\left(\right.$ knee extension $\left.\mathrm{PT}^{60^{\circ} / \mathrm{s}}\right)($ non-normalized, ratio standard and allometric scaling), and the correlation of muscle strength with body size.

\begin{tabular}{|c|c|c|c|c|c|c|c|c|c|c|c|c|c|c|}
\hline \multirow{2}{*}{ Variable } & \multirow{2}{*}{ Unit } & \multicolumn{5}{|c|}{ Men } & \multicolumn{5}{|c|}{ Women } & \multicolumn{3}{|c|}{$\begin{array}{l}\text { Correlation (r) } \\
\text { with body size }\end{array}$} \\
\hline & & AUC & $95 \% \mathrm{CI}$ & $\begin{array}{c}\text { Cut-off } \\
\text { point }(\leq)\end{array}$ & $\begin{array}{l}\text { Sens } \\
(\%)\end{array}$ & $\begin{array}{l}\text { Spe } \\
(\%)\end{array}$ & AUC & $95 \% \mathrm{CI}$ & $\begin{array}{c}\text { Cut-off } \\
\text { point }(\leq)\end{array}$ & $\begin{array}{l}\text { Sens } \\
(\%)\end{array}$ & $\begin{array}{l}\text { Spe } \\
(\%)\end{array}$ & $\begin{array}{l}\text { Body } \\
\text { mass }\end{array}$ & Height & $\begin{array}{c}\text { Variable for } \\
\text { normalization }\end{array}$ \\
\hline \multicolumn{15}{|l|}{$H G S(k g)$} \\
\hline Non-normalized & & $0.74^{*}$ & 0.548 to 0.886 & 36 & 86 & 59 & 0.61 & 0.477 to 0.725 & 22 & 52 & 70 & 0.17 & $0.39^{\S}$ & - \\
\hline /body mass & & 0.66 & 0.464 to 0.826 & 0.54 & 86 & 45 & $0.76^{\dagger}$ & 0.634 to 0.854 & 0.38 & 88 & 63 & $-0.57^{\S}$ & -0.03 & $-0.57^{\S}$ \\
\hline /body mass $\mathrm{s}^{0.22}$ & & $0.73^{*}$ & 0.538 to 0.880 & 14.4 & 86 & 59 & $0.65^{*}$ & 0.523 to 0.765 & 9.5 & 68 & 65 & -0.02 & $0.29^{\ddagger}$ & -0.02 \\
\hline /body mass ${ }^{0.40 ~(F O L E Y ~ e t ~ a l ., ~ 1999) ~}$ & & 0.70 & 0.504 to 0.856 & 6.7 & 86 & 50 & $0.68^{*}$ & 0.556 to 0.793 & 4.41 & 72 & 68 & -0.18 & 0.22 & -0.18 \\
\hline /body mass ${ }^{0.67 \text { (JARIC, 2003) }}$ & (kg) & 0.70 & 0.504 to 0.856 & 2.15 & 86 & 50 & $0.73^{*}$ & 0.607 to 0.833 & 1.46 & 80 & 65 & $-0.39^{\S}$ & 0.10 & $-0.39^{\S}$ \\
\hline /body mass 0.63 (PUA, 2006) & & 0.70 & 0.504 to 0.856 & 2.55 & 86 & 50 & $0.73^{*}$ & 0.600 to 0.829 & 1.77 & 84 & 60 & $-0.36^{\S}$ & 0.11 & $-0.36^{8}$ \\
\hline /body mass 0.31 (MARANHÃO NETO et al., 2017) & & $0.71^{*}$ & 0.517 to 0.865 & 10.9 & 100 & 41 & $0.67^{*}$ & 0.540 to 0.780 & 6.5 & 68 & 65 & -0.10 & $0.26^{\ddagger}$ & -0.10 \\
\hline /height & & 0.70 & 0.504 to 0.856 & 23.5 & 100 & 45 & 0.60 & 0.474 to 0.722 & 15.8 & 72 & 50 & 0.09 & 0.17 & 0.17 \\
\hline /height ${ }^{1.87}$ & (m) & 0.65 & 0.451 to 0.816 & 14.8 & 86 & 50 & 0.60 & 0.466 to 0.715 & 10.2 & 60 & 63 & 0.01 & -0.03 & -0.03 \\
\hline 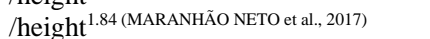 & & 0.65 & 0.451 to 0.816 & 15.1 & 86 & 50 & 0.60 & 0.467 to 0.716 & 10.4 & 60 & 63 & 0.01 & -0.02 & -0.02 \\
\hline /forearm circumference & & $0.74^{*}$ & 0.548 to 0.886 & 1.33 & 86 & 59 & $0.70^{*}$ & 0.570 to 0.805 & 1.04 & 84 & 58 & -0.17 & $0.27^{\ddagger}$ & -0.19 \\
\hline /forearm circumference $\mathrm{e}^{0.50}$ & & $0.75^{*}$ & 0.552 to 0.889 & 6.91 & 86 & 68 & $0.67^{*}$ & 0.537 to 0.777 & 4.84 & 68 & 65 & 0.01 & 0.34 & 0.02 \\
\hline /calf circumference & & $0.73^{*}$ & 0.531 to 0.875 & 0.92 & 57 & 82 & $0.67^{*}$ & 0.544 to 0.783 & 0.69 & 72 & 63 & -0.14 & $0.25^{\ddagger}$ & -0.21 \\
\hline /calf circumference ${ }^{0.54}$ & & $0.73^{*}$ & 0.531 to 0.875 & 5.35 & 86 & 59 & $0.64^{*}$ & 0.515 to 0.758 & 3.54 & 64 & 60 & 0.01 & $0.32^{\ddagger}$ & 0.01 \\
\hline /chest circumference & & 0.69 & 0.490 to 0.846 & 0.37 & 86 & 55 & $0.68^{*}$ & 0.551 to 0.789 & 0.28 & 84 & 58 & -0.20 & 0.24 & $-0.39^{\S}$ \\
\hline /chest circumference $e^{0.08}$ & $(\mathrm{~cm})$ & $0.75^{*}$ & 0.552 to 0.889 & 24.6 & 86 & 68 & 0.62 & 0.487 to 0.734 & 16.4 & 60 & 68 & 0.14 & $0.37^{\S}$ & -0.02 \\
\hline /knee height & & 0.71 & 0.510 to 0.861 & 0.72 & 100 & 45 & 0.61 & 0.478 to 0.726 & 0.44 & 44 & 75 & 0.05 & 0.19 & 0.03 \\
\hline$/$ knee height $^{1.32}$ & & 0.70 & 0.504 to 0.856 & 0.21 & 100 & 50 & 0.61 & 0.482 to 0.729 & 0.13 & 44 & 78 & 0.01 & 0.13 & -0.05 \\
\hline /half arm span & & 0.69 & 0.490 to 0.846 & 0.47 & 100 & 36 & 0.59 & 0.464 to 0.713 & 0.28 & 48 & 75 & 0.11 & 0.21 & 0.19 \\
\hline /half arm span ${ }^{1.81}$ & & 0.65 & 0.451 to 0.816 & 0.012 & 100 & 32 & 0.58 & 0.453 to 0.703 & 0.0086 & 72 & 45 & 0.04 & 0.04 & -0.03 \\
\hline /triceps skinfold & & $0.71^{*}$ & 0.514 to 0.863 & 2.25 & 86 & 59 & 0.65 & 0.519 to 0.762 & 0.93 & 64 & 68 & $-0.30^{\ddagger}$ & 0.15 & $-0.55^{\S}$ \\
\hline /biceps skinfold & & 0.63 & 0.428 to 0.798 & 6.67 & 100 & 36 & $0.73^{*}$ & 0.605 to 0.832 & 1.29 & 56 & 85 & $-0.35^{\S}$ & 0.16 & $-0.53^{8}$ \\
\hline /biceps skinfold ${ }^{-0.02}$ & & $0.75^{*}$ & 0.552 to 0.889 & 41.6 & 100 & 45 & 0.59 & 0.459 to 0.709 & 23.2 & 48 & 78 & $0.21^{\ddagger}$ & $0.38^{\S}$ & -0.05 \\
\hline /midaxillary skinfold & & 0.62 & 0.419 to 0.790 & 0.86 & 29 & 100 & 0.52 & 0.395 to 0.648 & 1.23 & 80 & 33 & -0.34 & 0.12 & $-0.65^{\S}$ \\
\hline /midaxillary skinfold ${ }^{-0.01}$ & & $0.75^{*}$ & 0.552 to 0.889 & 40.7 & 100 & 45 & 0.61 & 0.479 to 0.727 & 22.5 & 48 & 78 & 0.19 & $0.38^{\S}$ & -0.07 \\
\hline /pectoral skinfold & & 0.58 & 0.388 to 0.763 & 1.13 & 29 & 100 & $0.74^{\dagger}$ & 0.618 to 0.843 & 1.64 & 76 & 73 & $-0.31^{\ddagger}$ & $0.21^{*}$ & $-0.70^{\S}$ \\
\hline /suprailiac skinfold & (mm) & 0.61 & 0.413 to 0.785 & 1.88 & 71 & 55 & 0.62 & 0.488 to 0.735 & 0.77 & 64 & 73 & $-0.34^{\ddagger}$ & 0.12 & $-0.60^{\S}$ \\
\hline /suprailiac skinfold $^{0.01}$ & & $0.74^{*}$ & 0.545 to 0.884 & 35.3 & 86 & 68 & 0.62 & 0.486 to 0.733 & 23.4 & 64 & 68 & 0.17 & $0.38^{\S}$ & -0.07 \\
\hline /abdominal skinfold & & 0.66 & 0.464 to 0.826 & 1.13 & 57 & 73 & $0.69^{*}$ & 0.564 to 0.800 & 0.62 & 56 & 85 & $-0.29^{\ddagger}$ & 0.12 & $-0.58^{8}$ \\
\hline /thigh skinfold & & $0.78^{*}$ & 0.584 to 0.909 & 1.82 & 71 & 77 & $0.81^{\dagger}$ & 0.694 to 0.897 & 0.67 & 72 & 80 & -0.16 & $0.25^{\ddagger}$ & $-0.46^{\S}$ \\
\hline /medial calf skinfold & & $0.80^{\dagger}$ & 0.609 to 0.924 & 2.91 & 100 & 64 & $0.76^{\dagger}$ & 0.632 to 0.853 & 0.93 & 64 & 78 & -0.15 & 0.16 & $-0.42^{\S}$ \\
\hline /medial calf skinfold ${ }^{-0.03}$ & & $0.73^{*}$ & 0.538 to 0.880 & 39.1 & 86 & 59 & 0.59 & 0.457 to 0.706 & 24.5 & 52 & 70 & 0.21 & $0.38^{\S}$ & 0.04 \\
\hline /biacromial breadth & & 0.68 & 0.484 to 0.841 & 1.02 & 100 & 36 & 0.62 & 0.493 to 0.739 & 0.67 & 76 & 50 & 0.01 & $0.26^{\ddagger}$ & 0.01 \\
\hline /biacromial breadth ${ }^{1.06}$ & & 0.67 & 0.470 to 0.831 & 0.82 & 100 & 36 & 0.63 & 0.496 to 0.742 & 0.54 & 76 & 50 & 0.01 & $0.25^{\ddagger}$ & -0.01 \\
\hline /bitrochanteric breadth & $(\mathrm{mm})$ & 0.71 & 0.510 to 0.861 & 1.06 & 86 & 59 & $0.67^{*}$ & 0.538 to 0.778 & 0.7 & 68 & 60 & -0.02 & 0.29 & -0.05 \\
\hline /bitrochanteric breadth ${ }^{0.97}$ & & 0.71 & 0.510 to 0.861 & 1.18 & 86 & 59 & $0.67^{*}$ & 0.537 to 0.777 & 0.78 & 68 & 60 & -0.02 & 0.29 & -0.04 \\
\hline /bimalleolar breadth & & 0.65 & 0.454 to 0.819 & 6.25 & 100 & 32 & $0.66^{*}$ & 0.529 to 0.770 & 3.85 & 68 & 58 & 0.04 & $0.21^{+}$ & -0.10 \\
\hline
\end{tabular}




\begin{tabular}{|c|c|c|c|c|c|c|c|c|c|c|c|c|c|c|}
\hline \multirow{2}{*}{ Variable } & \multirow{2}{*}{ Unit } & \multicolumn{5}{|c|}{ Men } & \multicolumn{5}{|c|}{ Women } & \multicolumn{3}{|c|}{$\begin{array}{l}\text { Correlation (r) } \\
\text { with body size }\end{array}$} \\
\hline & & AUC & $95 \% \mathrm{CI}$ & $\begin{array}{c}\text { Cut-off } \\
\text { point }(\leq)\end{array}$ & $\begin{array}{c}\text { Sens } \\
(\%)\end{array}$ & $\begin{array}{l}\text { Spe } \\
(\%)\end{array}$ & AUC & 95\% CI & $\begin{array}{c}\text { Cut-off } \\
\text { point }(\leq)\end{array}$ & $\begin{array}{c}\text { Sens } \\
(\%)\end{array}$ & $\begin{array}{l}\text { Spe } \\
(\%)\end{array}$ & $\begin{array}{l}\text { Body } \\
\text { mass }\end{array}$ & Height & $\begin{array}{c}\text { Variable for } \\
\text { normalization }\end{array}$ \\
\hline /bimalleolar breadth ${ }^{0.91}$ & & 0.68 & 0.480 to 0.839 & 6.78 & 86 & 50 & $0.65^{*}$ & 0.525 to 0.767 & 4.51 & 68 & 58 & 0.05 & $0.23^{t}$ & -0.07 \\
\hline /elbow breadth & & 0.70 & 0.497 to 0.851 & 4.86 & 71 & 68 & $0.68^{*}$ & 0.550 to 0.788 & 4.18 & 76 & 58 & -0.04 & $0.31^{\ddagger}$ & $-0.26^{\ddagger}$ \\
\hline /elbow breadth ${ }^{0.37}$ & & $0.73^{*}$ & 0.531 to 0.875 & 20.4 & 100 & 41 & 0.64 & 0.506 to 0.751 & 12.1 & 64 & 65 & 0.10 & $0.36^{\S}$ & -0.03 \\
\hline /wrist breadth & & $0.80^{*}$ & 0.605 to 0.922 & 7.02 & 100 & 50 & 0.61 & 0.478 to 0.726 & 4.71 & 64 & 63 & 0.01 & $0.31^{\ddagger}$ & -0.19 \\
\hline /wrist breadth ${ }^{0.58}$ & & $0.76^{*}$ & 0.562 to 0.896 & 14.7 & 100 & 45 & 0.60 & 0.474 to 0.722 & 9.5 & 64 & 60 & 0.08 & $0.35^{\S}$ & -0.04 \\
\hline /chest breadth & & 0.67 & 0.470 to 0.831 & 1.32 & 100 & 45 & 0.60 & 0.466 to 0.716 & 0.88 & 64 & 63 & -0.03 & 0.28 & -0.20 \\
\hline /chest breadth ${ }^{0.34}$ & & $0.71^{*}$ & 0.517 to 0.865 & 11.5 & 86 & 59 & 0.61 & 0.476 to 0.725 & 7.53 & 56 & 70 & 0.04 & $0.31^{\ddagger}$ & -0.08 \\
\hline /body mass*height & & 0.60 & 0.400 to 0.774 & 0.17 & 29 & 100 & $0.74^{\dagger}$ & 0.616 to 0.841 & 0.23 & 76 & 68 & $-0.63^{\S}$ & -0.19 & $-0.60^{\S}$ \\
\hline$/(\text { body mass*height })^{0.26}$ & $(\mathrm{~kg} * \mathrm{~m})$ & 0.70 & 0.497 to 0.851 & 12 & 100 & 36 & $0.66^{*}$ & 0.533 to 0.774 & 7.63 & 76 & 55 & -0.08 & $0.23^{\ddagger}$ & -0.03 \\
\hline ISA & & 0.69 & 0.490 to 0.846 & 20.8 & 86 & 50 & $0.70^{*}$ & 0.574 to 0.807 & 14.1 & 80 & 63 & $-0.32^{\ddagger}$ & 0.08 & $-0.28^{\ddagger}$ \\
\hline$/ \mathrm{SA}^{0.49}$ & $\left(\mathrm{~m}^{2}\right)$ & 0.71 & 0.510 to 0.861 & 30.2 & 100 & 36 & $0.66^{*}$ & 0.529 to 0.770 & 18.3 & 68 & 63 & -0.07 & 0.24 & -0.02 \\
\hline /MAMC & & $0.77^{*}$ & 0.573 to 0.902 & 1.39 & 71 & 77 & $0.70^{*}$ & 0.568 to 0.803 & 1.02 & 64 & 78 & $-0.21^{\ddagger}$ & $0.23^{\ddagger}$ & $-0.37^{\S}$ \\
\hline$/$ MAMC $^{0.21}$ & (cm) & $0.75^{*}$ & 0.552 to 0.889 & 18.3 & 86 & 68 & 0.63 & 0.505 to 0.750 & 12.3 & 60 & 70 & 0.09 & $0.36^{\S}$ & 0.08 \\
\hline /CAMA & & 0.70 & 0.504 to 0.856 & 0.83 & 71 & 73 & $0.71^{*}$ & 0.588 to 0.819 & 0.66 & 60 & 80 & $-0.49^{\S}$ & 0.01 & $-0.74^{8}$ \\
\hline$/ \mathrm{CAMA}^{0.08}$ & & $0.75^{*}$ & 0.552 to 0.889 & 26.3 & 86 & 68 & 0.63 & 0.504 to 0.749 & 17.6 & 60 & 70 & 0.10 & $0.36^{\S}$ & 0.10 \\
\hline /AFA & $\left(\mathrm{cm}^{2}\right)$ & 0.71 & 0.517 to 0.865 & 2.17 & 86 & 55 & $0.70^{*}$ & 0.572 to 0.806 & 0.95 & 60 & 78 & $-0.42^{\S}$ & 0.08 & $-0.59^{\S}$ \\
\hline$/ \mathrm{AFA}^{0.01}$ & & $0.74^{*}$ & 0.545 to 0.884 & 34.9 & 86 & 68 & 0.63 & 0.497 to 0.743 & 23.2 & 60 & 70 & 0.16 & $0.38^{\S}$ & -0.01 \\
\hline$/$ FFM $_{\text {LEAN et al., 1996) }}$ & & 0.68 & 0.477 to 0.836 & 0.75 & 86 & 45 & $0.70^{*}$ & 0.577 to 0.810 & 0.68 & 84 & 63 & $-0.32^{\ddagger}$ & 0.06 & $-0.27^{\ddagger}$ \\
\hline$/$ FFM $^{0.53}\left(\right.$ LEAN et al, $^{1996)}$ & & 0.71 & 0.510 to 0.861 & 4.96 & 100 & 36 & $0.67^{*}$ & 0.544 to 0.783 & 3.63 & 76 & 58 & -0.09 & 0.22 & 0.01 \\
\hline /fat mass (LEAN et al., 1996) & (kg) & 0.64 & 0.438 to 0.806 & 2.69 & 100 & 32 & $0.76^{\dagger}$ & 0.638 to 0.857 & 0.8 & 76 & 78 & $-0.62^{\S}$ & -0.08 & $-0.63^{\S}$ \\
\hline /fat mass ${ }^{0.05}$ (LEAN et al., 1996) & & $0.75^{*}$ & 0.552 to 0.889 & 31.3 & 86 & 68 & 0.62 & 0.494 to 0.740 & 20.3 & 64 & 65 & 0.10 & $0.35^{\S}$ & 0.02 \\
\hline /left arm LST & & 0.53 & 0.333 to 0.713 & 0.01 & 29 & 100 & $0.69^{*}$ & 0.558 to 0.795 & 0.016 & 72 & 73 & $-0.42^{\S}$ & 0.01 & $-0.58^{\S}$ \\
\hline$/$ left arm LST T.29 & & 0.70 & 0.497 to 0.851 & 4.31 & 100 & 36 & $0.66^{*}$ & 0.528 to 0.769 & 2.93 & 72 & 63 & -0.02 & $0.29^{\ddagger}$ & 0.05 \\
\hline /right arm LST & & 0.56 & 0.363 to 0.741 & 0.012 & 57 & 73 & $0.70^{*}$ & 0.576 to 0.809 & 0.013 & 68 & 80 & $-0.4^{\S}$ & 0.06 & $-0.57^{\S}$ \\
\hline /right arm LST ${ }^{0.26}$ & & 0.70 & 0.497 to 0.851 & 4.21 & 71 & 68 & $0.66^{*}$ & 0.531 to 0.772 & 3.3 & 68 & 65 & 0.01 & $0.32^{\ddagger}$ & 0.08 \\
\hline /left leg LST & & 0.57 & 0.375 to 0.752 & 0.0027 & 29 & 100 & $0.65^{*}$ & 0.522 to 0.764 & 0.0041 & 60 & 75 & $-0.41^{\S}$ & -0.09 & $-0.50^{\S}$ \\
\hline$/$ left leg LST $^{0.30}$ & & 0.70 & 0.504 to 0.856 & 2.88 & 100 & 45 & 0.63 & 0.498 to 0.744 & 1.9 & 72 & 60 & -0.02 & $0.24^{\ddagger}$ & 0.01 \\
\hline /right leg LST & (g) & 0.53 & 0.333 to 0.713 & 0.0026 & 29 & 100 & $0.66^{*}$ & 0.530 to 0.771 & 0.0044 & 76 & 58 & $-0.37^{\S}$ & -0.01 & $-0.47^{\S}$ \\
\hline$/$ right leg LST ${ }^{0.30}$ & & 0.68 & 0.477 to 0.836 & 2.65 & 100 & 36 & 0.63 & 0.504 to 0.749 & 1.72 & 64 & 63 & 0.01 & $0.27^{\ddagger}$ & 0.05 \\
\hline /arms LST & & 0.54 & 0.345 to 0.725 & 0.0045 & 29 & 100 & $0.70^{*}$ & 0.573 to 0.807 & 0.0068 & 68 & 80 & $-0.42^{\S}$ & 0.04 & $-0.55^{\S}$ \\
\hline /arms LST ${ }^{0.30}$ & & 0.68 & 0.484 to 0.841 & 2.68 & 71 & 68 & $0.66^{*}$ & 0.528 to 0.769 & 2.09 & 64 & 68 & -0.03 & $0.30^{+}$ & 0.07 \\
\hline /legs LST & & 0.55 & 0.357 to 0.736 & 0.0013 & 29 & 100 & $0.65^{*}$ & 0.518 to 0.761 & 0.002 & 60 & 75 & $-0.39^{\S}$ & -0.05 & $-0.46^{8}$ \\
\hline$/ \operatorname{legs} \operatorname{LST}^{0.32}$ & & 0.68 & 0.477 to 0.836 & 1.91 & 100 & 36 & 0.64 & 0.506 to 0.751 & 1.25 & 68 & 63 & -0.02 & $0.25^{\ddagger}$ & 0.03 \\
\hline /ASM & & 0.54 & 0.345 to 0.725 & 1.03 & 29 & 100 & $0.66^{*}$ & 0.535 to 0.776 & 1.59 & 64 & 75 & $-0.41^{\S}$ & -0.03 & $-0.46^{\S}$ \\
\hline$/ \mathrm{ASM}^{0.33}$ & (kg) & 0.66 & 0.464 to 0.826 & 14.9 & 100 & 32 & $0.65^{*}$ & 0.517 to 0.760 & 9.87 & 72 & 63 & -0.04 & $0.25^{\ddagger}$ & 0.04 \\
\hline /ASM/height ${ }^{2}$ & & 0.67 & 0.467 to 0.829 & 4.74 & 71 & 68 & $0.67^{*}$ & 0.538 to 0.778 & 4.17 & 76 & 65 & $-0.23^{\ddagger}$ & 0.34 & $-0.50^{\S}$ \\
\hline /ASM/height ${ }^{(0.13)}$ & $\left(\mathrm{kg} / \mathrm{m}^{2}\right)$ & $0.73^{*}$ & 0.531 to 0.875 & 31.3 & 100 & 41 & 0.63 & 0.499 to 0.745 & 18.9 & 64 & 65 & 0.12 & $0.38^{\S}$ & 0.06 \\
\hline /FFM (BAUMGARTNER et al. 1991) & & 0.64 & 0.434 to 0.808 & 0.71 & 83 & 50 & $0.74^{\dagger}$ & 0.619 to 0.845 & 0.56 & 88 & 59 & $-0.4^{\S}$ & 0.10 & $-0.39^{\S}$ \\
\hline$/ \mathrm{FFM}^{0.41}$ (BAUMGARTNER et al., 1991) & & 0.69 & 0.488 to 0.849 & 7.11 & 83 & 59 & $0.68^{*}$ & 0.546 to 0.787 & 5.29 & 84 & 51 & -0.07 & $0.28^{\ddagger}$ & -0.02 \\
\hline$/ \mathrm{FFM}_{(\mathrm{DXA})}$ & & 0.60 & 0.406 to 0.779 & 0.41 & 29 & 100 & $0.73^{*}$ & 0.600 to 0.829 & 0.65 & 84 & 63 & $-0.43^{\S}$ & -0.04 & $-0.44^{\S}$ \\
\hline$/ \mathrm{FFM}^{0.36}(\mathrm{DXA})$ & (kg) & 0.70 & 0.504 to 0.856 & 10.1 & 100 & 36 & $0.65^{*}$ & 0.526 to 0.768 & 6.71 & 72 & 60 & -0.06 & 0.24 & 0.02 \\
\hline /fat mass $(\mathrm{DXA})$ & & 0.71 & 0.517 to 0.865 & 1.01 & 43 & 95 & $0.77^{\dagger}$ & 0.643 to 0.861 & 0.9 & 80 & 68 & $-0.53^{\S}$ & 0.03 & $-0.66^{\S}$ \\
\hline /fat mass ${ }^{0.03}(\mathrm{DXA})$ & & $0.74^{*}$ & 0.545 to 0.884 & 32.3 & 86 & 68 & 0.62 & 0.490 to 0.737 & 21.3 & 60 & 65 & 0.13 & $0.37^{\S}$ & -0.04 \\
\hline
\end{tabular}




\begin{tabular}{|c|c|c|c|c|c|c|c|c|c|c|c|c|c|c|}
\hline \multirow{2}{*}{ Variable } & \multirow{2}{*}{ Unit } & \multicolumn{5}{|c|}{ Men } & \multicolumn{5}{|c|}{ Women } & \multicolumn{3}{|c|}{$\begin{array}{l}\text { Correlation (r) } \\
\text { with body size }\end{array}$} \\
\hline & & AUC & 95\% CI & $\begin{array}{c}\text { Cut-off } \\
\text { point }(\leq)\end{array}$ & $\begin{array}{l}\text { Sens } \\
(\%)\end{array}$ & $\begin{array}{l}\text { Spe } \\
(\%)\end{array}$ & AUC & 95\% CI & $\begin{array}{c}\text { Cut-off } \\
\text { point }(\leq)\end{array}$ & $\begin{array}{l}\text { Sens } \\
(\%)\end{array}$ & $\begin{array}{l}\text { Spe } \\
(\%)\end{array}$ & $\begin{array}{l}\text { Body } \\
\text { mass }\end{array}$ & Height & $\begin{array}{c}\text { Variable for } \\
\text { normalization }\end{array}$ \\
\hline Non-normalized & \multirow{6}{*}{ (kg) } & $0.75^{*}$ & 0.555 to 0.891 & 56.1 & 86 & 77 & $0.67^{*}$ & 0.545 to 0.784 & 38.1 & 72 & 60 & 0.18 & $0.33^{\ddagger}$ & \\
\hline /body mass & & $0.77^{*}$ & 0.580 to 0.907 & 0.85 & 86 & 68 & $0.76^{\dagger}$ & 0.634 to 0.854 & 0.54 & 68 & 78 & $-0.28^{\ddagger}$ & 0.13 & $-0.28^{\ddagger}$ \\
\hline /body mass ${ }^{0.44}$ & & $0.75^{*}$ & 0.559 to 0.893 & 9.04 & 86 & 77 & $0.71^{*}$ & 0.586 to 0.818 & 6.03 & 76 & 63 & -0.03 & $0.24^{\ddagger}$ & -0.03 \\
\hline /body mass ${ }^{0.67 \text { (JARIC, 2003) }}$ & & $0.78^{*}$ & 0.587 to 0.911 & 3.4 & 86 & 77 & $0.73^{\dagger}$ & 0.604 to 0.832 & 2.28 & 76 & 65 & -0.13 & 0.20 & -0.13 \\
\hline /body mass ${ }^{0.96}$ (ABDALLA et al., 2020) & & $0.77^{*}$ & 0.573 to 0.902 & 1.00 & 86 & 68 & $0.75^{\dagger}$ & 0.629 to 0.851 & 0.45 & 44 & 100 & $-0.26^{\ddagger}$ & 0.13 & $-0.26^{\ddagger}$ \\
\hline /body mass ${ }^{0.69}$ (ABDALLA et al., 2020) & & $0.78^{*}$ & 0.587 to 0.911 & 3.06 & 86 & 77 & $0.73^{*}$ & 0.606 to 0.833 & 1.48 & 44 & 98 & -0.15 & 0.19 & -0.15 \\
\hline /height & \multirow[b]{2}{*}{ (m) } & $0.73^{*}$ & 0.531 to 0.875 & 33 & 86 & 77 & $0.69^{*}$ & 0.584 to 0.779 & 33 & 88 & 48 & 0.12 & $0.21^{\ddagger}$ & $0.21^{\ddagger}$ \\
\hline height $^{3.05}(\mathrm{~m})$ & & 0.68 & 0.484 to 0.841 & 12.2 & 86 & 64 & $0.68^{*}$ & 0.557 to 0.794 & 8.44 & 60 & 83 & 0.02 & -0.03 & -0.03 \\
\hline /forearm circumference & \multirow{12}{*}{$(\mathrm{cm})$} & $0.75^{*}$ & 0.552 to 0.889 & 2.16 & 86 & 77 & $0.70^{*}$ & 0.577 to 0.810 & 1.38 & 60 & 75 & 0.01 & $0.27^{\ddagger}$ & -0.16 \\
\hline /forearm circumference $e^{0.38}$ & & $0.75^{*}$ & 0.552 to 0.889 & 16.1 & 86 & 77 & $0.69^{*}$ & 0.560 to 0.796 & 11.2 & 76 & 58 & 0.11 & $0.3^{\ddagger}$ & -0.02 \\
\hline /calf circumference & & $0.74^{*}$ & 0.545 to 0.884 & 1.65 & 86 & 77 & $0.70^{*}$ & 0.573 to 0.807 & 1.06 & 72 & 68 & 0.02 & $0.26^{\ddagger}$ & -0.02 \\
\hline /calf circumference ${ }^{1.10}$ & & $0.75^{*}$ & 0.552 to 0.889 & 1.14 & 86 & 77 & $0.71^{*}$ & 0.580 to 0.813 & 0.7 & 68 & 73 & 0.01 & $0.26^{\ddagger}$ & -0.04 \\
\hline /chest circumference & & $0.76^{*}$ & 0.566 to 0.898 & 0.64 & 86 & 73 & $0.71^{*}$ & 0.580 to 0.813 & 0.4 & 72 & 65 & -0.03 & $0.26^{\ddagger}$ & -0.15 \\
\hline /chest circumference ${ }^{0.56}$ & & $0.75^{*}$ & 0.552 to 0.889 & 4.62 & 86 & 77 & $0.69^{*}$ & 0.566 to 0.801 & 3.06 & 76 & 60 & 0.06 & $0.29^{\ddagger}$ & -0.05 \\
\hline /waist circumference & & $0.78^{*}$ & 0.587 to 0.911 & 0.73 & 100 & 59 & $0.72^{*}$ & 0.591 to 0.821 & 0.37 & 60 & 80 & -0.11 & $0.26^{\ddagger}$ & -0.24 \\
\hline /waist circumference ${ }^{0.28}$ & & $0.75^{*}$ & 0.552 to 0.889 & 16.5 & 86 & 77 & $0.69^{*}$ & 0.563 to 0.799 & 11.4 & 76 & 58 & 0.09 & $0.31^{\ddagger}$ & 0.01 \\
\hline /knee height & & $0.73^{*}$ & 0.538 to 0.880 & 1.02 & 86 & 77 & $0.68^{*}$ & 0.554 to 0.792 & 0.78 & 76 & 60 & 0.10 & $0.22^{\ddagger}$ & 0.15 \\
\hline$/ \mathrm{knee} \mathrm{height}^{2.28}$ & & 0.71 & 0.510 to 0.861 & 0.0065 & 86 & 77 & $0.68^{*}$ & 0.554 to 0.792 & 0.0041 & 52 & 83 & 0.01 & 0.09 & $0.24^{\ddagger}$ \\
\hline /half arm span & & $0.72^{*}$ & 0.524 to 0.870 & 0.65 & 86 & 77 & $0.68^{*}$ & 0.547 to 0.786 & 0.48 & 76 & 60 & 0.13 & $0.23^{\ddagger}$ & $0.26^{\ddagger}$ \\
\hline /half arm span ${ }^{2.73}$ & & 0.64 & 0.438 to 0.806 & 0.48 & 76 & 60 & $0.67^{*}$ & 0.545 to 0.784 & 0.00026 & 76 & 58 & 0.05 & 0.05 & 0.14 \\
\hline /triceps skinfold & \multirow{8}{*}{$(\mathrm{mm})$} & $0.81^{\dagger}$ & 0.624 to 0.932 & 4.22 & 86 & 68 & $0.70^{*}$ & 0.575 to 0.809 & 1.4 & 60 & 75 & $-0.24^{\ddagger}$ & $0.26^{\ddagger}$ & 0.07 \\
\hline /triceps skinfold ${ }^{0.18}$ & & $0.77^{*}$ & 0.580 to 0.907 & 36.5 & 86 & 77 & $0.69^{*}$ & 0.564 to 0.799 & 21.6 & 76 & 60 & 0.09 & $0.34^{\ddagger}$ & 0.10 \\
\hline /biceps skinfold & & 0.71 & 0.507 to 0.858 & 7.24 & 71 & 68 & $0.76^{\dagger}$ & 0.637 to 0.856 & 2.5 & 80 & 65 & $-0.25^{\ddagger}$ & 0.24 & $-0.38^{\S}$ \\
\hline /biceps skinfold ${ }^{0.08}$ & & $0.77^{*}$ & 0.573 to 0.902 & 48.9 & 86 & 77 & $0.69^{*}$ & 0.566 to 0.801 & 31.3 & 76 & 60 & 0.12 & $0.32^{\ddagger}$ & -0.17 \\
\hline /thigh skinfold & & $0.81^{*}$ & 0.624 to 0.932 & 2.95 & 71 & 86 & $0.79^{\dagger}$ & 0.668 to 0.879 & 1.18 & 76 & 73 & -0.09 & $0.31^{\ddagger}$ & $-0.26^{\ddagger}$ \\
\hline /thigh skinfold ${ }^{0.03}$ & & $0.75^{*}$ & 0.559 to 0.893 & 51.1 & 86 & 77 & $0.68^{*}$ & 0.553 to 0.790 & 34.1 & 72 & 63 & 0.16 & $0.33^{\ddagger}$ & -0.04 \\
\hline /medial calf skinfold & & $0.83^{\dagger}$ & 0.646 to 0.944 & 4.61 & 86 & 73 & $0.77^{\dagger}$ & 0.646 to 0.863 & 1.6 & 76 & 70 & -0.09 & $0.23^{\ddagger}$ & $-0.38^{\S}$ \\
\hline /medial calf skinfold ${ }^{0.02}$ & & $0.75^{*}$ & 0.559 to 0.893 & 53.6 & 86 & 77 & $0.68^{*}$ & 0.551 to 0.789 & 37.1 & 76 & 60 & 0.17 & $0.32^{\ddagger}$ & -0.02 \\
\hline /biacromial breadth & \multirow{12}{*}{$(\mathrm{mm})$} & $0.73^{*}$ & 0.531 to 0.875 & 1.42 & 86 & 77 & $0.68^{*}$ & 0.552 to 0.789 & 1.07 & 76 & 58 & 0.08 & $0.27^{\ddagger}$ & 0.13 \\
\hline /biacromial breadth ${ }^{1.67}$ & & 0.71 & 0.510 to 0.861 & 0.12 & 86 & 77 & $0.68^{*}$ & 0.550 to 0.788 & 0.11 & 84 & 50 & 0.02 & $0.22^{\ddagger}$ & $0.26^{\ddagger}$ \\
\hline /bitrochanteric breadth & & $0.73^{*}$ & 0.538 to 0.880 & 1.72 & 86 & 77 & $0.70^{*}$ & 0.572 to 0.806 & 1.16 & 76 & 60 & 0.08 & $0.27^{\ddagger}$ & 0.01 \\
\hline /bitrochanteric breadth ${ }^{0.69}$ & & $0.74^{*}$ & 0.545 to 0.884 & 5.1 & 86 & 77 & $0.69^{*}$ & 0.567 to 0.802 & 3.48 & 76 & 60 & 0.11 & $0.28^{\ddagger}$ & -0.04 \\
\hline /bimalleolar breadth & & $0.73^{*}$ & 0.531 to 0.875 & 8.77 & 86 & 77 & $0.70^{*}$ & 0.575 to 0.808 & 5.77 & 76 & 65 & 0.10 & $0.24 \ddagger$ & 0.12 \\
\hline /bimalleolar breadth ${ }^{1.20}$ & & $0.71^{*}$ & 0.517 to 0.865 & 6.01 & 86 & 68 & $0.70^{*}$ & 0.577 to 0.810 & 3.93 & 76 & 65 & 0.08 & 0.22 & 0.13 \\
\hline /elbow breadth & & $0.73^{*}$ & 0.538 to 0.880 & 9.36 & 86 & 73 & $0.70^{*}$ & 0.569 to 0.804 & 6.57 & 76 & 63 & 0.05 & $0.28^{\ddagger}$ & -0.09 \\
\hline /elbow breadth ${ }^{0.10}$ & & $0.75^{*}$ & 0.559 to 0.893 & 47.2 & 86 & 77 & $0.68^{*}$ & 0.548 to 0.786 & 32.7 & 76 & 60 & 0.16 & $0.32 \ddagger$ & -0.10 \\
\hline /wrist breadth & & $0.79^{*}$ & 0.602 to 0.919 & 11.5 & 100 & 64 & $0.66^{*}$ & 0.536 to 0.776 & 7.33 & 72 & 58 & 0.07 & $0.29^{\ddagger}$ & -0.05 \\
\hline /wrist breadth ${ }^{0.44}$ & & $0.75^{*}$ & 0.559 to 0.893 & 26.1 & 86 & 77 & $0.67^{*}$ & 0.542 to 0.782 & 18.4 & 72 & 63 & 0.13 & $0.31^{\ddagger}$ & 0.03 \\
\hline /chest breadth & & $0.73^{*}$ & 0.538 to 0.880 & 1.85 & 86 & 77 & $0.66^{*}$ & 0.532 to 0.773 & 1.44 & 76 & 55 & 0.05 & $0.27^{\ddagger}$ & 0.02 \\
\hline /chest breadth ${ }^{0.68}$ & & $0.73^{*}$ & 0.531 to 0.875 & 5.45 & 86 & 77 & $0.66^{*}$ & 0.536 to 0.776 & 4.06 & 76 & 58 & 0.09 & $0.29^{\ddagger}$ & 0.06 \\
\hline /body mass*height & \multirow{2}{*}{$(\mathrm{kg} * \mathrm{~m})$} & $0.73^{*}$ & 0.538 to 0.880 & 0.52 & 86 & 64 & $0.75^{\dagger}$ & 0.627 to 0.849 & 0.33 & 68 & 78 & $-0.33^{\ddagger}$ & 0.02 & 0.10 \\
\hline$/(\text { body mass*height })^{0.48}$ & & $0.75^{*}$ & 0.552 to 0.889 & 5.83 & 86 & 77 & $0.72^{*}$ & 0.589 to 0.820 & 4.06 & 76 & 65 & -0.07 & 0.18 & 0.14 \\
\hline /SA & \multirow[b]{2}{*}{$\left(\mathrm{m}^{2}\right)$} & $0.75^{*}$ & 0.552 to 0.889 & 31.6 & 86 & 77 & $0.72^{*}$ & 0.593 to 0.823 & 21.2 & 72 & 68 & -0.09 & 0.18 & $-0.29^{\ddagger}$ \\
\hline$/ \mathrm{SA}^{0.93}$ & & $0.75^{*}$ & 0.559 to 0.893 & 33 & 86 & 77 & $0.72^{*}$ & 0.589 to 0.820 & 22.7 & 76 & 65 & -0.07 & 0.19 & -0.03 \\
\hline /MAMC & $(\mathrm{cm})$ & $0.77^{*}$ & 0.573 to 0.902 & 2.42 & 86 & 73 & $0.72^{*}$ & 0.592 to 0.822 & 1.54 & 64 & 75 & -0.03 & $0.26^{\ddagger}$ & -0.11 \\
\hline
\end{tabular}




\begin{tabular}{|c|c|c|c|c|c|c|c|c|c|c|c|c|c|c|}
\hline \multirow{2}{*}{ Variable } & \multirow{3}{*}{ Unit } & \multicolumn{5}{|c|}{ Men } & \multicolumn{5}{|c|}{ Women } & \multicolumn{3}{|c|}{$\begin{array}{l}\text { Correlation (r) } \\
\text { with body size }\end{array}$} \\
\hline & & AUC & $95 \%$ CI & $\begin{array}{c}\text { Cut-off } \\
\text { point }(\leq)\end{array}$ & $\begin{array}{l}\text { Sens } \\
(\%)\end{array}$ & $\begin{array}{l}\text { Spe } \\
(\%)\end{array}$ & AUC & $95 \%$ CI & $\begin{array}{c}\text { Cut-off } \\
\text { point }(\leq)\end{array}$ & $\begin{array}{l}\text { Sens } \\
(\%)\end{array}$ & $\begin{array}{l}\text { Spe } \\
(\%)\end{array}$ & $\begin{array}{l}\text { Body } \\
\text { mass }\end{array}$ & Height & $\begin{array}{c}\text { Variable for } \\
\text { normalization }\end{array}$ \\
\hline /MAMC $^{0.18}$ & & $0.75^{*}$ & 0.559 to 0.893 & 32 & 86 & 77 & $0.68^{*}$ & 0.557 to 0.794 & 22.5 & 76 & 60 & 0.14 & $0.31^{t}$ & -0.09 \\
\hline /CAMA & \multirow[b]{2}{*}{$\left(\mathrm{cm}^{2}\right)$} & $0.71^{*}$ & 0.517 to 0.865 & 2.03 & 100 & 41 & $0.73^{*}$ & 0.602 to 0.830 & 0.9 & 52 & 93 & $-0.26^{\ddagger}$ & 0.14 & -0.24 \\
\hline$/ \mathrm{CAMA}^{0.08}$ & & $0.75^{*}$ & 0.559 to 0.893 & 41.7 & 86 & 77 & $0.69^{*}$ & 0.558 to 0.795 & 28.5 & 72 & 63 & 0.13 & $0.31^{\ddagger}$ & 0.04 \\
\hline$/ \mathrm{FFM}_{\text {(LEAN et al., 1996) }}$ & \multirow{2}{*}{$(\mathrm{kg})$} & $0.76^{*}$ & 0.566 to 0.898 & 1.11 & 86 & 77 & $0.72^{*}$ & 0.592 to 0.822 & 1 & 72 & 68 & -0.09 & 0.18 & -0.18 \\
\hline$/ \mathrm{FFM}^{0.88}{ }_{\text {(LEAN et al., 1996) }}$ & & $0.75^{*}$ & 0.559 to 0.893 & 1.77 & 86 & 77 & $0.71^{*}$ & 0.583 to 0.815 & 1.53 & 76 & 65 & -0.06 & 0.20 & 0.20 \\
\hline /left arm LST & \multirow{12}{*}{ (g) } & 0.64 & 0.438 to 0.806 & 0.026 & 86 & 59 & $0.70^{*}$ & 0.568 to 0.803 & 0.021 & 52 & 88 & -0.18 & 0.14 & 0.05 \\
\hline$/$ left arm LST ${ }^{0.26}$ & & $0.72^{*}$ & 0.524 to 0.870 & 7.77 & 86 & 77 & $0.69^{*}$ & 0.560 to 0.796 & 5.86 & 72 & 63 & 0.08 & $0.28^{\ddagger}$ & 0.07 \\
\hline /right arm LST & & 0.62 & 0.425 to 0.795 & 0.026 & 100 & 45 & $0.70^{*}$ & 0.578 to 0.811 & 0.013 & 40 & 98 & -0.17 & 0.18 & $-0.30^{\ddagger}$ \\
\hline /right arm LST ${ }^{0.22}$ & & $0.73^{*}$ & 0.538 to 0.880 & 10.1 & 86 & 77 & $0.69^{*}$ & 0.559 to 0.795 & 7.44 & 72 & 63 & 0.09 & $0.3^{\ddagger}$ & 0.07 \\
\hline /left leg LST & & 0.68 & 0.477 to 0.836 & 0.0079 & 86 & 64 & $0.68^{*}$ & 0.553 to 0.790 & 0.0048 & 44 & 93 & -0.18 & 0.08 & -0.15 \\
\hline$/$ left leg LST $^{0.64}$ & & $0.72^{*}$ & 0.524 to 0.870 & 0.19 & 86 & 77 & $0.69^{*}$ & 0.560 to 0.796 & 0.12 & 44 & 88 & -0.05 & 0.17 & 0.19 \\
\hline /right leg LST & & 0.65 & 0.451 to 0.816 & 0.0079 & 86 & 55 & $0.69^{*}$ & 0.561 to 0.797 & 0.005 & 44 & 93 & -0.15 & 0.13 & -0.14 \\
\hline /right leg $\mathrm{LST}^{0.58}$ & & $0.73^{*}$ & 0.531 to 0.875 & 0.33 & 86 & 77 & $0.69^{*}$ & 0.562 to 0.798 & 0.21 & 48 & 83 & -0.01 & $0.21^{\ddagger}$ & 0.01 \\
\hline /arms LST & & 0.63 & 0.432 to 0.801 & 0.012 & 86 & 55 & $0.70^{*}$ & 0.572 to 0.806 & 0.009 & 52 & 85 & -0.18 & 0.17 & -0.18 \\
\hline /arms $\mathrm{LST}^{0.26}$ & & $0.72^{*}$ & 0.524 to 0.870 & 6.38 & 86 & 77 & $0.69^{*}$ & 0.561 to 0.797 & 4.76 & 72 & 63 & 0.08 & $0.29^{\sharp}$ & 0.01 \\
\hline /legs LST & & 0.68 & 0.477 to 0.836 & 0.0039 & 86 & 59 & $0.68^{*}$ & 0.556 to 0.793 & 0.0024 & 44 & 93 & -0.16 & 0.10 & -0.17 \\
\hline$/ \operatorname{legs} \operatorname{LST}^{0.64}$ & & $0.73^{*}$ & 0.531 to 0.875 & 0.12 & 86 & 77 & $0.69^{*}$ & 0.559 to 0.795 & 0.08 & 44 & 88 & -0.04 & 0.18 & 0.18 \\
\hline /ASM & \multirow[b]{2}{*}{$(\mathrm{kg})$} & 0.66 & 0.457 to 0.821 & 2.99 & 86 & 55 & $0.69^{*}$ & 0.561 to 0.797 & 1.74 & 44 & 93 & -0.17 & 0.12 & -0.17 \\
\hline$/ \mathrm{ASM}^{0.56}$ & & $0.72^{*}$ & 0.524 to 0.870 & 10.7 & 86 & 77 & $0.69^{*}$ & 0.565 to 0.801 & 5.68 & 36 & 98 & -0.02 & $0.21^{\ddagger}$ & -0.01 \\
\hline /ASM/height ${ }^{2}$ & \multirow[b]{2}{*}{$\left(\mathrm{kg} / \mathrm{m}^{2}\right)$} & $0.73^{*}$ & 0.538 to 0.880 & 8.47 & 86 & 73 & $0.70^{*}$ & 0.568 to 0.803 & 4.88 & 48 & 88 & -0.06 & 0.34 & $-0.28^{\ddagger}$ \\
\hline /ASM/height ${ }^{2^{(0.23)}}$ & & $0.73^{*}$ & 0.538 to 0.880 & 36.3 & 86 & 77 & $0.68^{*}$ & 0.554 to 0.791 & 22.4 & 60 & 73 & 0.12 & $0.33^{\ddagger}$ & -0.10 \\
\hline /FFM (BAUMGARTNER et al., 1991) & \multirow{4}{*}{$(\mathrm{kg})$} & $0.78^{*}$ & 0.584 to 0.914 & 1.13 & 83 & 73 & $0.75^{\dagger}$ & 0.620 to 0.845 & 0.83 & 76 & 64 & -0.14 & 0.20 & -0.03 \\
\hline$/ \mathrm{FFM}^{0.67}{ }_{\text {(BAUMGARTNER et al., 1991) }}$ & & $0.76^{*}$ & 0.559 to 0.898 & 3.94 & 83 & 77 & $0.72^{*}$ & 0.592 to 0.823 & 2.91 & 76 & 64 & -0.03 & 0.24 & 0.16 \\
\hline$/ \mathrm{FFM}_{(\mathrm{DXA})}$ & & 0.70 & 0.504 to 0.856 & 1.16 & 86 & 73 & $0.70^{*}$ & 0.575 to 0.808 & 0.67 & 44 & 93 & -0.17 & 0.11 & -0.08 \\
\hline$/ \mathrm{FFM}^{0.38}$ (DXA) & & $0.72^{*}$ & 0.524 to 0.870 & 12.7 & 86 & 77 & $0.69^{*}$ & 0.562 to 0.798 & 9.48 & 72 & 60 & 0.04 & $0.24^{\ddagger}$ & 0.02 \\
\hline \multicolumn{15}{|c|}{ knee extension $P T^{60 \%}(\mathrm{Nm})$} \\
\hline Non-normalized & & $0.93^{\dagger}$ & 0.769 to 0.991 & 85.4 & 86 & 95 & $0.74^{\dagger}$ & 0.619 to 0.843 & 66.6 & 64 & 75 & 0.20 & $0.42^{\S}$ & - \\
\hline /body mass & \multirow{6}{*}{$(\mathrm{kg})$} & $0.90^{\dagger}$ & 0.734 to 0.981 & 1.26 & 86 & 91 & $0.84^{\dagger}$ & 0.726 to 0.918 & 0.93 & 72 & 88 & $-0.32^{\ddagger}$ & 0.17 & $-0.32^{\ddagger}$ \\
\hline /body mass ${ }^{0.37}$ & & $0.93^{\dagger}$ & 0.769 to 0.991 & 18.2 & 86 & 95 & $0.80^{\dagger}$ & 0.677 to 0.885 & 16.2 & 80 & 68 & 0.01 & 0.33 & 0.01 \\
\hline /body mass 0.67 (DAVIES; DALSKY, 1997) & & $0.93^{\dagger}$ & 0.769 to 0.991 & 5.06 & 86 & 95 & $0.82^{\dagger}$ & 0.704 to 0.904 & 3.71 & 68 & 88 & -0.15 & $0.26^{\ddagger}$ & -0.15 \\
\hline /body mass ${ }^{0.72 ~(D A V I E S ; ~ D A L S K Y, ~ 1997) ~}$ & & $0.94^{\dagger}$ & 0.778 to 0.993 & 4.1 & 86 & 95 & $0.82^{\dagger}$ & 0.710 to 0.907 & 3.14 & 72 & 85 & -0.18 & 0.24 & -0.18 \\
\hline /body mass ${ }^{0.74(D A V I E S ; ~ D A L S K Y, ~ 1997) ~}$ & & $0.93^{\dagger}$ & 0.769 to 0.991 & 3.77 & 86 & 95 & $0.82^{\dagger}$ & 0.710 to 0.907 & 2.87 & 72 & 85 & -0.19 & 0.24 & -0.19 \\
\hline /body mass. 0.67 (JARIC, 2003) & & $0.93^{\dagger}$ & 0.769 to 0.991 & 5.06 & 86 & 95 & $0.82^{\dagger}$ & 0.704 to 0.904 & 3.71 & 68 & 88 & -0.15 & $0.26^{\ddagger}$ & -0.15 \\
\hline /height & \multirow[b]{2}{*}{ (m) } & $0.93^{\dagger}$ & 0.769 to 0.991 & 54.1 & 86 & 95 & $0.74^{\dagger}$ & 0.620 to 0.843 & 44.1 & 64 & 75 & 0.14 & $0.30^{\ddagger}$ & $0.30^{\ddagger}$ \\
\hline /height $^{3.27}$ & & $0.86^{\dagger}$ & 0.677 to 0.958 & 19.2 & 100 & 77 & $0.74^{\dagger}$ & 0.617 to 0.842 & 17.4 & 72 & 65 & 0.01 & 0.01 & 0.01 \\
\hline /forearm circumference & \multirow{9}{*}{$(\mathrm{cm})$} & $0.94^{\dagger}$ & 0.787 to 0.995 & 3.88 & 100 & 82 & $0.80^{\dagger}$ & 0.683 to 0.890 & 2.59 & 60 & 88 & 0.01 & $0.36^{\S}$ & -0.09 \\
\hline /forearm circumference ${ }^{0.59}$ & & $0.95^{\dagger}$ & 0.796 to 0.996 & 14.9 & 100 & 86 & $0.78^{\dagger}$ & 0.664 to 0.876 & 12.8 & 92 & 55 & 0.08 & $0.38^{\S}$ & 0.02 \\
\hline /calf circumference & & $0.90^{\dagger}$ & 0.734 to 0.981 & 2.46 & 86 & 91 & $0.79^{\dagger}$ & 0.676 to 0.884 & 2.13 & 84 & 65 & 0.02 & 0.34 & -0.01 \\
\hline /calf circumference $e^{1.18}$ & & $0.90^{\dagger}-$ & 0.734 to 0.981 & 1.31 & 86 & 91 & $0.80^{\dagger}$ & 0.686 to 0.891 & 1.2 & 88 & 63 & -0.01 & 0.32 & -0.05 \\
\hline /chest circumference & & $0.90^{\dagger}$ & 0.726 to 0.978 & 0.9 & 86 & 91 & $0.79^{\dagger}$ & 0.676 to 0.884 & 0.67 & 64 & 83 & -0.03 & 0.34 & -0.12 \\
\hline /chest circumference $^{0.72}$ & & $0.90^{\dagger}$ & 0.734 to 0.981 & 3.16 & 86 & 91 & $0.78^{\dagger}$ & 0.663 to 0.876 & 2.27 & 60 & 85 & 0.03 & $0.36^{\S}$ & -0.04 \\
\hline /waist circumference & & $0.90^{\dagger}$ & 0.726 to 0.978 & 0.93 & 86 & 91 & $0.79^{\dagger}$ & 0.670 to 0.880 & 0.71 & 68 & 83 & -0.13 & $0.33^{\ddagger}$ & $-0.23^{\ddagger}$ \\
\hline /waist circumference ${ }^{0.40}$ & & $0.93^{\dagger}$ & 0.769 to 0.991 & 13.8 & 86 & 95 & $0.77^{\dagger}$ & 0.648 to 0.865 & 11.4 & 72 & 73 & 0.07 & $0.38^{\S}$ & -0.01 \\
\hline /knee height & & $0.94^{\dagger}$ & 0.778 to 0.993 & 1.83 & 100 & 86 & $0.75^{\dagger}$ & 0.624 to 0.847 & 1.44 & 68 & 73 & 0.12 & $0.30^{\ddagger}$ & 0.14 \\
\hline
\end{tabular}




\begin{tabular}{|c|c|c|c|c|c|c|c|c|c|c|c|c|c|c|}
\hline \multirow{2}{*}{ Variable } & \multirow{2}{*}{ Unit } & \multicolumn{5}{|c|}{ Men } & \multicolumn{5}{|c|}{ Women } & \multicolumn{3}{|c|}{$\begin{array}{l}\text { Correlation (r) } \\
\text { with body size }\end{array}$} \\
\hline & & AUC & 95\% CI & $\begin{array}{c}\text { Cut-off } \\
\text { point }(\leq)\end{array}$ & $\begin{array}{c}\text { Sens } \\
(\%)\end{array}$ & $\begin{array}{l}\text { Spe } \\
(\%)\end{array}$ & AUC & $95 \% \mathrm{CI}$ & $\begin{array}{c}\text { Cut-off } \\
\text { point }(\leq)\end{array}$ & $\begin{array}{l}\text { Sens } \\
(\%)\end{array}$ & $\begin{array}{l}\text { Spe } \\
(\%)\end{array}$ & $\begin{array}{l}\text { Body } \\
\text { mass }\end{array}$ & Height & $\begin{array}{c}\text { Variable for } \\
\text { normalization }\end{array}$ \\
\hline$/$ knee height $^{1.82}$ & & $0.90^{\dagger}$ & 0.734 to 0.981 & 0.068 & 100 & 86 & $0.74^{\dagger}$ & 0.617 to 0.842 & 0.053 & 64 & 75 & 0.05 & $0.21^{t}$ & 0.02 \\
\hline /half arm span & & $0.91^{\dagger}$ & 0.743 to 0.984 & 1.04 & 86 & 91 & $0.75^{\dagger}$ & 0.627 to 0.849 & 0.9 & 68 & 70 & 0.15 & $0.31^{\ddagger}$ & 0.11 \\
\hline /half arm span ${ }^{1.62}$ & & $0.88^{\dagger}$ & 0.709 to 0.972 & 0.076 & 100 & 73 & $0.75^{\dagger}$ & 0.631 to 0.852 & 0.066 & 84 & 58 & 0.11 & 0.24 & 0.01 \\
\hline /triceps skinfold & & $0.86^{\dagger}$ & 0.677 to 0.958 & 5.26 & 86 & 86 & $0.78^{\dagger}$ & 0.660 to 0.873 & 2.45 & 64 & 85 & $-0.25^{\ddagger}$ & $0.26^{\ddagger}$ & $-0.57^{\S}$ \\
\hline /triceps skinfold ${ }^{0.15}$ & & $0.93^{\dagger}$ & 0.769 to 0.991 & 55.6 & 86 & 95 & $0.76^{\dagger}$ & 0.641 to 0.860 & 47.6 & 80 & 63 & 0.12 & $0.42^{\S}$ & -0.12 \\
\hline /biceps skinfold & & $0.79^{*}$ & 0.602 to 0.919 & 16.8 & 100 & 59 & $0.82^{\dagger}$ & 0.704 to 0.904 & 5.3 & 92 & 63 & $-0.27^{\ddagger}$ & $0.25^{\ddagger}$ & $-0.56^{8}$ \\
\hline /biceps skinfold ${ }^{0.06}$ & & $0.93^{\dagger}$ & 0.769 to 0.991 & 76.1 & 86 & 95 & $0.75^{\dagger}$ & 0.631 to 0.852 & 71.9 & 88 & 53 & 0.16 & $0.41^{\S}$ & -0.14 \\
\hline /thigh skinfold & (mm) & $0.92^{\dagger}$ & 0.751 to 0.986 & 5.31 & 100 & 86 & $0.82^{\dagger}$ & 0.708 to 0.907 & 1.85 & 72 & 83 & -0.11 & 0.32 & $-0.57^{\S}$ \\
\hline /thigh skinfold $^{-0.10}$ & & $0.90^{\dagger}$ & 0.734 to 0.981 & 115 & 86 & 91 & $0.73^{\dagger}$ & 0.601 to 0.829 & 105 & 76 & 60 & $0.23^{\ddagger}$ & $0.39^{\S}$ & -0.06 \\
\hline /medial calf skinfold & & $0.92^{\dagger}$ & 0.760 to 0.988 & 7.21 & 86 & 95 & $0.83^{\dagger}$ & 0.720 to 0.914 & 2.5 & 72 & 85 & -0.10 & $0.25^{\ddagger}$ & $-0.51^{\S}$ \\
\hline /medial calf skinfold ${ }^{0.003}$ & & $0.93^{\dagger}$ & 0.769 to 0.991 & 84.8 & 86 & 95 & $0.74^{\dagger}$ & 0.620 to 0.843 & 66 & 64 & 75 & 0.20 & $0.41^{\S}$ & -0.04 \\
\hline /biacromial breadth & & $0.90^{\dagger}$ & 0.726 to 0.978 & 2.56 & 100 & 73 & $0.76^{\dagger}$ & 0.642 to 0.861 & 2.04 & 84 & 60 & 0.1 & $0.35^{\S}$ & 0.15 \\
\hline /biacromial breadth ${ }^{2.15}$ & & $0.82^{\dagger}$ & 0.631 to 0.936 & 0.038 & 86 & 68 & $0.77^{\dagger}$ & 0.647 to 0.864 & 0.03 & 76 & 73 & -0.01 & $0.27^{\star}$ & -0.04 \\
\hline /bitrochanteric breadth & & $0.93^{\dagger}$ & 0.769 to 0.991 & 2.56 & 86 & 95 & $0.79^{\dagger}$ & 0.666 to 0.877 & 2.13 & 76 & 73 & 0.08 & $0.35^{\S}$ & -0.08 \\
\hline /bitrochanteric breadth ${ }^{0.50}$ & & $0.93^{\dagger}$ & 0.769 to 0.991 & 14.8 & 86 & 95 & $0.77^{\dagger}$ & 0.647 to 0.864 & 14.1 & 88 & 58 & 0.14 & $0.38^{\S}$ & 0.01 \\
\hline /bimalleolar breadth & & $0.88^{\dagger}$ & 0.701 to 0.969 & 13.6 & 86 & 86 & $0.79^{\dagger}$ & 0.666 to 0.877 & 12.0 & 84 & 65 & 0.10 & $0.31^{\ddagger}$ & 0.03 \\
\hline /bimalleolar breadth ${ }^{1.54}$ & & $0.81^{*}$ & 0.616 to 0.928 & 5.04 & 86 & 73 & $0.80^{\dagger}$ & 0.681 to 0.888 & 4.64 & 92 & 60 & 0.06 & $0.26^{\ddagger}$ & -0.07 \\
\hline /elbow breadth & (mm) & $0.90^{\dagger}$ & 0.726 to 0.978 & 14 & 86 & 86 & $0.79^{\dagger}$ & 0.674 to 0.884 & 13.9 & 88 & 63 & 0.07 & $0.37^{\S}$ & -0.20 \\
\hline /elbow breadth ${ }^{0.10}$ & & $0.93^{\dagger}$ & 0.769 to 0.991 & 70.6 & 86 & 95 & $0.75^{\dagger}$ & 0.624 to 0.847 & 56.6 & 64 & 75 & 0.18 & $0.41^{\S}$ & 0.01 \\
\hline /wrist breadth & & $0.93^{\dagger}$ & 0.769 to 0.991 & 15.9 & 86 & 95 & $0.76^{\dagger}$ & 0.636 to 0.856 & 14.2 & 68 & 73 & 0.09 & $0.38^{\S}$ & -0.07 \\
\hline /wrist breadth ${ }^{0.80}$ & & $0.93^{\dagger}$ & 0.769 to 0.991 & 22.1 & 86 & 95 & $0.76^{\dagger}$ & 0.635 to 0.855 & 19.7 & 72 & 73 & 0.11 & $0.38^{\S}$ & -0.03 \\
\hline /chest breadth & & $0.89^{\dagger}$ & 0.718 to 0.975 & 3.33 & 100 & 73 & $0.75^{\dagger}$ & 0.625 to 0.848 & 2.2 & 56 & 83 & 0.07 & $0.37^{\S}$ & 0.05 \\
\hline /chest breadth ${ }^{1.40}$ & & $0.88^{\dagger}$ & 0.701 to 0.969 & 0.85 & 100 & 73 & $0.74^{\dagger}$ & 0.611 to 0.837 & 0.77 & 84 & 55 & 0.02 & 0.34 & -0.04 \\
\hline /body mass*height & & $0.84^{\dagger}$ & 0.654 to 0.947 & 0.8 & 86 & 77 & $0.84^{\dagger}$ & 0.729 to 0.920 & 0.6 & 72 & 88 & $-0.38^{8}$ & 0.04 & $-0.32^{\ddagger}$ \\
\hline$/(\text { body mass* } * \text { height })^{0.4}$ & $(\mathrm{~kg} * \mathrm{~m})$ & $0.94^{\dagger}$ & 0.787 to 0.995 & 13 & 100 & 82 & $0.80^{\dagger}$ & 0.681 to 0.888 & 10.5 & 84 & 65 & -0.06 & $0.26^{\ddagger}$ & 0.01 \\
\hline$/\left(\right.$ body mass*height) ${ }^{0.97}$ (SEGAL et al., 2008) & & $0.86^{\dagger}$ & 0.677 to 0.958 & 0.9 & 86 & 77 & $0.84^{\dagger}$ & 0.729 to 0.920 & 0.68 & 72 & 88 & $-0.37^{\S}$ & 0.05 & $-0.31^{\ddagger}$ \\
\hline /SA & & $0.94^{\dagger}$ & 0.787 to 0.995 & 56.9 & 100 & 82 & $0.81^{\dagger}$ & 0.692 to 0.896 & 36.3 & 64 & 88 & -0.10 & $0.24^{\ddagger}$ & -0.04 \\
\hline$/ \mathrm{SA}^{0.83}$ & $\left(\mathrm{~m}^{2}\right)$ & $0.94^{\dagger}$ & 0.778 to 0.993 & 53.8 & 86 & 95 & $0.80^{\dagger}$ & 0.680 to 0.887 & 50 & 84 & 65 & -0.05 & $0.28^{\ddagger}$ & 0.01 \\
\hline /MAMC & & $0.95^{\dagger}$ & 0.796 to 0.996 & 4.11 & 100 & 86 & $0.80^{\dagger}$ & 0.677 to 0.885 & 2.82 & 68 & 88 & -0.04 & 0.34 & $-0.21^{\ddagger}$ \\
\hline$/ \mathrm{MAMC}^{0.25}$ & (cm) & $0.94^{\dagger}$ & 0.778 to 0.993 & 45.9 & 100 & 82 & $0.77^{\dagger}$ & 0.643 to 0.861 & 32.9 & 72 & 70 & 0.14 & $0.40^{\S}$ & 0.08 \\
\hline /CAMA & & $0.96^{\dagger}$ & 0.806 to 0.998 & 2.66 & 100 & 82 & $0.80^{\dagger}$ & 0.685 to 0.891 & 2.49 & 84 & 73 & $-0.31^{\ddagger}$ & 0.18 & $-0.58^{8}$ \\
\hline$/$ CAMA $^{0.11}$ & $\left(\mathrm{~cm}^{2}\right)$ & $0.94^{\dagger}$ & 0.778 to 0.993 & 68.5 & 100 & 82 & $0.77^{\dagger}$ & 0.643 to 0.861 & 48.7 & 72 & 70 & 0.14 & $0.40^{\S}$ & 0.10 \\
\hline$/$ FFM $_{\text {LEAN et al.. 1996) }}$ & 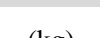 & $0.93^{\dagger}$ & 0.769 to 0.991 & 1.79 & 86 & 95 & $0.81^{\dagger}$ & 0.697 to 0.899 & 1.84 & 76 & 80 & -0.11 & $0.23^{\ddagger}$ & -0.10 \\
\hline$/ \mathrm{FFM}^{0.53}$ (LEAN et al., 1996) & (Kg) & $0.94^{\dagger}$ & 0.778 to 0.993 & 10.7 & 86 & 95 & $0.79^{\dagger}$ & 0.674 to 0.884 & 11.2 & 84 & 65 & 0.04 & 0.32 & 0.06 \\
\hline /left arm LST & & $0.81^{*}$ & 0.616 to 0.928 & 0.047 & 86 & 68 & $0.76^{\dagger}$ & 0.641 to 0.860 & 0.047 & 76 & 75 & -0.20 & 0.19 & $-0.35^{\S}$ \\
\hline$/$ left arm LST ${ }^{0.32}$ & & $0.91^{\dagger}$ & 0.743 to 0.984 & 7.65 & 86 & 91 & $0.77^{\dagger}$ & 0.646 to 0.863 & 6.72 & 72 & 70 & 0.07 & $0.36^{\S}$ & 0.09 \\
\hline /right arm LST & & $0.80^{*}$ & 0.609 to 0.924 & 0.034 & 71 & 86 & $0.78^{\dagger}$ & 0.662 to 0.875 & 0.036 & 68 & 83 & -0.18 & $0.21^{\ddagger}$ & $-0.44^{\S}$ \\
\hline /right arm LST ${ }^{0.09}$ & & $0.93^{\dagger}$ & 0.769 to 0.991 & 41.8 & 86 & 95 & $0.76^{\dagger}$ & 0.635 to 0.855 & 38.8 & 80 & 63 & 0.16 & $0.40^{\S}$ & 0.10 \\
\hline /left leg LST & & $0.82^{\dagger}$ & 0.631 to 0.936 & 0.015 & 100 & 55 & $0.76^{\dagger}$ & 0.636 to 0.856 & 0.012 & 68 & 80 & -0.19 & 0.13 & $-0.25^{\ddagger}$ \\
\hline$/$ left leg LST $^{0.43}$ & (g) & $0.92^{\dagger}$ & 0.760 to 0.988 & 2.26 & 100 & 77 & $0.77^{\dagger}$ & 0.648 to 0.865 & 1.83 & 76 & 70 & 0.03 & $0.30^{+}$ & 0.05 \\
\hline /right leg LST & & $0.77^{*}$ & 0.580 to 0.907 & 0.015 & 100 & 50 & $0.78^{\dagger}$ & 0.662 to 0.875 & 0.013 & 76 & 78 & -0.16 & 0.18 & -0.21 \\
\hline /right leg LST ${ }^{0.48}$ & & $0.90^{\dagger}$ & 0.734 to 0.981 & 1.39 & 100 & 73 & $0.78^{\dagger}$ & 0.656 to 0.870 & 1.16 & 80 & 70 & 0.02 & $0.31^{\ddagger}$ & 0.05 \\
\hline /arms LST & & $0.81^{*}$ & 0.616 to 0.928 & 0.018 & 71 & 82 & $0.78^{\dagger}$ & 0.656 to 0.870 & 0.022 & 76 & 78 & -0.19 & 0.21 & $-0.38^{\S}$ \\
\hline /arms LST ${ }^{0.21}$ & & $0.92^{\dagger}$ & 0.751 to 0.986 & 14.8 & 86 & 95 & $0.77^{\dagger}$ & 0.651 to 0.867 & 14.0 & 80 & 63 & 0.11 & $0.38^{\S}$ & 0.10 \\
\hline /legs LST & & $0.81^{*}$ & 0.616 to 0.928 & 0.0063 & 71 & 82 & $0.77^{\dagger}$ & 0.651 to 0.867 & 0.0065 & 76 & 78 & -0.18 & 0.15 & $-0.21^{\ddagger}$ \\
\hline
\end{tabular}




\begin{tabular}{|c|c|c|c|c|c|c|c|c|c|c|c|c|c|c|}
\hline \multirow{2}{*}{ Variable } & \multirow{2}{*}{ Unit } & \multicolumn{5}{|c|}{ Men } & \multicolumn{5}{|c|}{ Women } & \multicolumn{3}{|c|}{$\begin{array}{l}\text { Correlation (r) } \\
\text { with body size }\end{array}$} \\
\hline & & AUC & 95\% CI & $\begin{array}{c}\text { Cut-off } \\
\text { point }(\leq)\end{array}$ & $\begin{array}{l}\text { Sens } \\
(\%)\end{array}$ & $\begin{array}{l}\text { Spe } \\
(\%)\end{array}$ & AUC & 95\% CI & $\begin{array}{c}\text { Cut-off } \\
\text { point ( } \leq)\end{array}$ & $\begin{array}{l}\text { Sens } \\
(\%)\end{array}$ & $\begin{array}{l}\text { Spe } \\
(\%)\end{array}$ & $\begin{array}{l}\text { Body } \\
\text { mass }\end{array}$ & Height & $\begin{array}{c}\text { Variable for } \\
\text { normalization }\end{array}$ \\
\hline$/$ legs LST ${ }^{0.47}$ & & $0.92^{\dagger}$ & 0.751 to 0.986 & 1.07 & 100 & 73 & $0.77^{\dagger}$ & 0.652 to 0.868 & 0.87 & 76 & 70 & 0.02 & $0.30^{\ddagger}$ & 0.05 \\
\hline /ASM & & $0.81^{*}$ & 0.624 to 0.932 & 4.66 & 71 & 86 & $0.77^{\dagger}$ & 0.652 to 0.868 & 5.01 & 76 & 78 & -0.18 & 0.17 & $-0.23^{\ddagger}$ \\
\hline$/ \mathrm{ASM}^{0.42}$ & (kg) & $0.91^{\dagger}$ & 0.743 to 0.984 & 27.2 & 86 & 86 & $0.77^{\dagger}$ & 0.646 to 0.863 & 23.9 & 76 & 70 & 0.03 & $0.32^{\ddagger}$ & 0.07 \\
\hline /ASM/height ${ }^{2}$ & & $0.88^{\dagger}$ & 0.701 to 0.969 & 11.9 & 71 & 95 & $0.78^{\dagger}$ & 0.656 to 0.870 & 11.5 & 72 & 78 & -0.06 & $0.39^{\S}$ & $-0.35^{8}$ \\
\hline /ASM/height ${ }^{2(0.02)}$ & $\left(\mathrm{kg} / \mathrm{m}^{2}\right)$ & $0.93^{\dagger}$ & 0.769 to 0.991 & 82.3 & 86 & 95 & $0.74^{\dagger}$ & 0.620 to 0.843 & 64.6 & 64 & 75 & 0.19 & $0.41^{8}$ & 0.06 \\
\hline /FFM (BAUMGARTNER et al., 1991) & & $0.93^{\dagger}$ & 0.769 to 0.992 & 1.63 & 83 & 95 & $0.85^{\dagger}$ & 0.734 to 0.924 & 1.6 & 88 & 77 & -0.16 & $0.25^{\ddagger}$ & -0.15 \\
\hline$/$ FFM $^{0.61}$ (BAUMGARTNER et al., 1991) & & $0.92^{\dagger}$ & 0.759 to 0.990 & 7.54 & 83 & 95 & $0.81^{\dagger}$ & 0.694 to 0.898 & 7.2 & 88 & 67 & -0.02 & $0.32^{\ddagger}$ & 0.01 \\
\hline$/ \mathrm{FFM}_{(\mathrm{DXA})}$ & (kg) & $0.84^{\dagger}$ & 0.654 to 0.947 & 2.08 & 100 & 59 & $0.79^{\dagger}$ & 0.672 to 0.882 & 1.89 & 76 & 75 & -0.19 & 0.17 & $-0.23^{\ddagger}$ \\
\hline$/ \mathrm{FFM}^{0.32}{ }_{(\mathrm{DXA})}$ & & $0.93^{\dagger}$ & 0.769 to 0.991 & 26.1 & 86 & 95 & $0.77^{\dagger}$ & 0.652 to 0.868 & 25.9 & 88 & 55 & 0.07 & $0.34^{\ddagger}$ & 0.09 \\
\hline
\end{tabular}

${ }^{*} \mathrm{p}<0.05$ and ${ }^{\dagger} \mathrm{p}<0.001$ (statistically significant AUC)

${ }_{\mathrm{p}}^{\mathrm{t}}<0.05$ and ${ }^{\S} \mathrm{p}<0.001$ (statistically significant correlation)

Dependent variable (primary outcome): functional limitation $(6 \mathrm{MWT}<400 \mathrm{~m})$

Note: $\mathrm{AUC}=$ area under the curve; $\mathrm{CI}=$ confidence interval; $p=$ significance; Sens=sensibility; Spe=specificity; $\mathrm{SA}=$ surface area of human body; MAMC=mid-arm muscle circumference; $\mathrm{CAMA}=$ corrected arm muscle area; $\mathrm{AFA}=$ arm fat area; FFM=Fat-free mass; LST=lean soft tissue; ASM=appendicular skeletal muscle mass; DXA=Dual-energy X-ray absorptiometry; 6MWT=six-minute walk test. 